\title{
Nonlinear viscoelastodynamic equations of three-dimensional rotating structures in finite displacement and finite element discretization.
}

\author{
C. Desceliers, C. Soize \\ Laboratory of Engineering Mechanics, University of Marne-La-Vallée, France
}

\section{Full postal address:}

Christian Soize

Laboratoire de Mécanique

Université de Marne-la-Vallée

5 , boulevard Descartes

77454 Marne-La-Vallée Cedex 2

France

E-mail address : soize@univ-mlv.fr

\begin{abstract}
This paper deals with the nonlinear viscoelastodynamics of three-dimensional rotating structure undergoing finite displacement. In addition, the nonlinear dynamics is studied with respect to geometrical and mechanical perturbations. On part of the boundary of the structure, a rigid body displacement field is applied which moves the structure in a rotation motion. A time dependent Dirichlet condition is applied to another part of the boundary. For instance, this corresponds to the cycle step of a helicopter rotor blade. A surface force field is applied to a third part of the boundary and depends on the time history of the structural displacement field. For example, this might corresponds to general unsteady aerodynamics forces applied to the structure. The objective of this paper is to model the nonlinear dynamic behavior of such a rotating viscoelastic structure undergoing finite displacements, and to allow small geometrical and mechanical (mass, constitutive equations) perturbations analysis to be performed. The model is constructed by the introduction of a reference configuration which is deduced from the nonlinear steady boundary value problem. A constitutive equation deduced from the Coleman and Noll theory concerning the viscoelasticity in finite displacement is used. Thereafter, the weak formulation of the boundary value problem is constructed and discretized using the finite element method. In order to simplify the mathematical study of the equations, multilinear forms are introduced in the algebraic calculation and their mathematical properties are presented.
\end{abstract}

Keywords : Nonlinear Structural Dynamics, Rotating Structure, Viscoelasticity in Finite Displacement, Sensitivity Analysis. 


\section{Introduction.}

Vibration analysis of rotating structures is an important subject in mechanical engineering. This field has been extensively studied in particular for linear vibration problems (for instance, see the non exhaustive list of references, [ $3,16,19,21,24,28,44,45,46,48,49])$. Sometimes, nonlinear vibration analysis of rotating structures has to be performed because of the presence of finite displacement of the structure. This can be the case for rotating structures constituted of slender parts, such as for certain helicopter rotor blades. Many publications devoted to nonlinear vibrations of solids are based on the use of nonlinear beam and plate theories (for instance, see $[10,18,36,47]$ ). It should be noted that, if the use of such approximated theories are very efficient for isotropic materials having no significant viscoelastic effects, some difficulties can arise for a structure in finite displacement constituted of nonisotropic viscoelastic material (for instance, this is the case of certain advanced helicopter rotor technology made of composite materials). This paper is devoted to nonlinear dynamics of rotating structures constituted of nonisotropic viscoelastic three-dimensional bodies in finite displacement, for which complex loads and complex Dirichlet boundary conditions are considered, and for which a very little exists in the literature. In the other hand, it should be noted that many works have been published concerning viscoelastic constitutive equations (for linearized theory, see for instance $[6,20,31,38,43]$ and for nonlinear theory with finite displacement, see [13]) and concerning the use of viscoelastic models in vibration problems (see for instance $[4,33,38,40,42,47])$. Concerning the constitutive model, physically nonlinear theory should be mentioned (see for instance [8]). In this theory, a nonlinear relation between the Cauchy stress tensor and the linearized deformation tensor is constructed, preserving invariance under superposed rigid body motions by writing the constitutive equations in the local reference frame of a material point of the structure rather than in a global reference frame. Other invariant theories use this kind of strategy to study invariant infinitesimal motions of elastic body (see for instance [9], [39]). Such theories could be used to study small or moderate vibrations around a given finite deformation (see also [22]). Nevertheless, it should be noted that this paper is not concerned with infinitesimal motion superposed to a given deformation.

This paper presents the nonlinear viscoelastodynamic equations of three-dimensional rotating structures in finite displacement without introducing any approximation in order to allow sensitivity analysis to be numerically performed. On a first part of the boundary of the structure considered, a rigid body displacement field operates to move the structure in a rotation motion. A time dependent Dirichlet 
condition concerning the structural displacement field is applied to a second part of the boundary of the structure (for instance, in the context of helicopter rotor blades, this time dependent Dirichlet condition corresponds to the cyclic step which allows the aerodynamic loads to be adapted). A surface force field depending on the time history of the structural displacement field is applied to a third part of the boundary of the structure (for instance, such a surface force field corresponds to general unsteady aerodynamics forces). We present the constitutive equations of the viscoelasticity in finite displacement (deduced from the Coleman and Noll theory [13]) in the rotating frame and with respect to the steady configuration. The important objective of this paper is to deduce the numerical formulation based on the use of the finite element method $[2,12,14,23,50]$ to analyze nonlinear responses of the dynamical system for small data perturbations to the geometric and the mechanical parameters. In this context, weak formulations $[7,11,14,17,37,38,41]$ of the boundary value problems are systematically introduced in order to deduce the matrix equations resulting from a finite element discretization. Taking into account that we are concerned with three-dimensional structures, several formulations could be constructed (each corresponding to several possible choices of different reference configurations). In this paper, we present a strategy to analyze such a mechanical system. This consists in constructing a steady problem corresponding to the structure in equilibrium under prestresses and steady loads in the rotating frame (for instance, the time average part of unsteady body forces). It should be noted that this configuration does not correspond to a given motion, but has to be constructed. We choose the deformed structure associated with the steady equilibrium as the reference configuration. The nonlinear viscoelastodynamic equations are transported on the reference configuration, because the nonlinear dynamical responses about the steady configuration in the rotating frame are of prime importance. For instance, such a formulation allows linearization of the dynamics to be performed in order to study linear instabilities of the structure coupled with unsteady aerodynamic flow but, in the present paper, we are not interested in such a linearized theory. A treatment by translation of the time dependent Dirichlet conditions is presented for this kind of nonlinear viscoelastodynamic system. Finally, a complete algebraic developement is presented which allow sensitivity analysis and random analysis to be numerically performed without any kinematical approximations. As a result of such a strategy, among others and due to the specific treatment used for the time dependent Dirichlet condition, nonlinear integrodifferential matrix equations with periodic coefficients are deduced which can be used for a parametric analysis. 


\section{Nonlinear boundary value problem of a rotating structure in a fixed reference frame}

Let $\mathcal{R}_{0}$ be the fixed reference frame whose origin is the point $O$ which is assumed to be located on the axis of rotation of the structure. In $\mathcal{R}_{0}$ and at time $t$, the deformed configuration occupies a bounded domain which is denoted by $\widetilde{\Omega}(t) \subset \mathbb{R}^{3}$ with boundary $\partial \widetilde{\Omega}(t)$. It is assumed that boundary $\partial \widetilde{\Omega}(t)$ is sufficiently smooth. In $\mathcal{R}_{0}$ and at time $s$, let $\widetilde{\mathbf{X}}\left(t, s, \widetilde{\mathbf{x}}_{t}\right) \in \widetilde{\Omega}(s)$ be the position of a particle which is located in $\mathcal{R}_{0}$ and at time $t>s$ at position $\widetilde{\mathbf{x}}_{t} \in \widetilde{\Omega}(t)$. Let $s \mapsto \widetilde{\mathbf{w}}\left(t, s, \widetilde{\mathbf{x}}_{t}\right)$ be the $\mathbb{R}^{3}$-valued displacement field time history of domain $\widetilde{\Omega}(t)$ defined on $]-\infty, t]$, such that

$$
\widetilde{\mathbf{w}}\left(t, s, \widetilde{\mathbf{x}}_{t}\right)=\widetilde{\mathbf{X}}\left(t, s, \widetilde{\mathbf{x}}_{t}\right)-\widetilde{\mathbf{x}}_{t}
$$

In $\mathcal{R}_{0}$ and at time $t$, the rotating structure is subject to an external surface force field $\widetilde{\mathbf{F}}\left(t, \widetilde{\mathbf{x}}_{t}\right)$ applied to the part $\widetilde{\Sigma}(t)$ of boundary $\partial \widetilde{\Omega}(t)$ and to an external body force field $\widetilde{\rho}\left(t, \widetilde{\mathbf{x}}_{t}\right) \widetilde{\mathbf{f}}\left(t, \widetilde{\mathbf{x}}_{t}\right)$ applied in $\widetilde{\Omega}(t)$ in which $\widetilde{\rho}\left(t, \widetilde{\mathbf{x}}_{t}\right)$ is the mass density of $\widetilde{\Omega}(t)$. In order to model general loads (for instance unsteady aerodynamic loads which depend on the time history of the structural displacement field), we write $\widetilde{\mathbf{F}}\left(t, \widetilde{\mathbf{x}}_{t}\right)=\int_{0}^{+\infty} \widetilde{\mathbf{G}}\left(\tau, t, \widetilde{\mathbf{x}}_{t},\{\widetilde{\mathbf{w}}(t, t-\tau, \widetilde{\mathbf{y}})\}_{\tilde{\mathbf{y}} \in \tilde{\Sigma}(t)}\right) d \tau$, in which for all $\widetilde{\mathbf{x}}_{t} \in \widetilde{\Sigma}(t)$ and for any functions $\delta \widetilde{\mathbf{w}}$ from $\widetilde{\Sigma}(t)$ into $\mathbb{R}^{3}$, function $\tau \mapsto \widetilde{\mathbf{G}}\left(\tau, t, \widetilde{\mathbf{x}}_{t}, \delta \widetilde{\mathbf{w}}\right)$ with values in $\mathbb{R}^{3}$ is integrable on $] 0,+\infty[$. The notation $\{\widetilde{\mathbf{w}}(t, t-\tau, \widetilde{\mathbf{y}})\}_{\tilde{\mathbf{y}} \in \tilde{\Sigma}(t)}$ denotes the mapping $\widetilde{\mathbf{y}} \mapsto \widetilde{\mathbf{w}}(t, t-\tau, \widetilde{\mathbf{y}})$ from $\widetilde{\Sigma}(t)$ into $\mathbb{R}^{3}$. On a second part $\widetilde{\Upsilon}(t)$ of the boundary $\partial \widetilde{\Omega}(t)$, we impose a rigid-body displacement field defined by the rotation associated with a direct orthogonal $(3 \times 3)$ real matrix $[Q(t)]$. On a third part $\widetilde{\Gamma}(t)$ of boundary $\partial \widetilde{\Omega}(t)$, a time dependent Dirichlet condition related to the displacement field $\widetilde{\mathbf{w}}\left(t, s, \widetilde{\mathbf{x}}_{t}\right)$ is applied and defined by $\widetilde{\mathbf{w}}\left(t, s, \widetilde{\mathbf{x}}_{t}\right)=\widetilde{\mathbf{w}}^{\operatorname{dir}}\left(t, s, \widetilde{\mathbf{x}}_{t}\right)$. Let $\widetilde{\widetilde{\sigma}}\left(t, \widetilde{\mathbf{x}}_{t}\right)$ be the Cauchy stress tensor related to $\widetilde{\Omega}(t)$. Therefore, in $\mathcal{R}_{0}$ and at time $t$, the equations of the three-dimensional rotating structure are

$$
\begin{aligned}
& \forall \widetilde{\mathbf{x}}_{t} \in \widetilde{\Omega}(t), \quad \operatorname{div}_{\tilde{\mathbf{x}}_{t}} \widetilde{\sigma}\left(t, \widetilde{\mathbf{x}}_{t}\right)+\widetilde{\rho}\left(t, \widetilde{\mathbf{x}}_{t}\right) \widetilde{\mathbf{f}}\left(t, \widetilde{\mathbf{x}}_{t}\right)=\widetilde{\rho}\left(t, \widetilde{\mathbf{x}}_{t}\right)\left\{\frac{\partial^{2} \widetilde{\mathbf{w}}}{\partial s^{2}}\left(t, s, \widetilde{\mathbf{x}}_{t}\right)\right\}_{s=t}, \\
& \forall \widetilde{\mathbf{x}}_{t} \in \widetilde{\Sigma}(t), \quad \widetilde{\widetilde{\sigma}}\left(t, \widetilde{\mathbf{x}}_{t}\right) \widetilde{\mathbf{n}}\left(t, \widetilde{\mathbf{x}}_{t}\right)=\widetilde{\mathbf{F}}\left(t, \widetilde{\mathbf{x}}_{t}\right), \\
& \forall \widetilde{\mathbf{x}}_{t} \in \widetilde{\Upsilon}(t), \quad \widetilde{\mathbf{w}}\left(t, s, \widetilde{\mathbf{x}}_{t}\right)=\left\{[Q(s)][Q(t)]^{\mathrm{T}}-[I]\right\} \widetilde{\mathbf{x}}_{t}, \\
& \forall \widetilde{\mathbf{x}}_{t} \in \widetilde{\Gamma}(t), \quad \widetilde{\mathbf{w}}\left(t, s, \widetilde{\mathbf{x}}_{t}\right)=\widetilde{\mathbf{w}}^{\operatorname{dir}}\left(t, s, \widetilde{\mathbf{x}}_{t}\right), \\
& \text { with } \quad \widetilde{\mathbf{F}}\left(t, \widetilde{\mathbf{x}}_{t}\right)=\int_{0}^{+\infty} \widetilde{\mathbf{G}}\left(\tau, t, \widetilde{\mathbf{x}}_{t},\{\widetilde{\mathbf{w}}(t, t-\tau, \widetilde{\mathbf{y}})\}_{\tilde{\mathbf{y}} \in \tilde{\Sigma}(t)}\right) d \tau,
\end{aligned}
$$

and where $\widetilde{\mathbf{n}}\left(t, \widetilde{\mathbf{x}}_{t}\right) \in \mathbb{R}^{3}$ is the unit outward normal vector to the boundary $\partial \widetilde{\Omega}(t)$, the exponent $T$ means the transpose of a matrix and $[I]$ is the $(3 \times 3)$ identity matrix. 


\section{Nonlinear dynamical boundary value problem of a rotating structure in a rotating frame.}

Let $\mathcal{R}_{1}$ be the rotating reference frame attached to the rotating structure and let $\left(\mathbf{e}_{0,1}, \mathbf{e}_{0,2}, \mathbf{e}_{0,3}\right)$ denote the canonical basis of $\mathbb{R}^{3}$. Rotating frame $\mathcal{R}_{1}$ is defined by the origin $O$ and the direct orthonormal basis $\left(\mathbf{e}_{1,1}(t), \mathbf{e}_{1,2}(t), \mathbf{e}_{1,3}(t)\right)$ which is derived from $\left(\mathbf{e}_{0,1}, \mathbf{e}_{0,2}, \mathbf{e}_{0,3}\right)$ by the rotation represented by matrix $[Q(t)]$ and such that $\mathbf{e}_{1, p}(t)=[Q(t)] \mathbf{e}_{0, p}$ for any $p$ fixed in $\{1,2,3\}$. Any particle located at position $\widetilde{\mathbf{y}}$ in $\mathcal{R}_{0}$ at time $t$ is located at position $\mathbf{y}=\mathbf{X}_{t}(\widetilde{\mathbf{y}})$ in $\mathcal{R}_{1}$ where $\mathbf{X}_{t}(\widetilde{\mathbf{y}})=[Q(t)]^{T} \widetilde{\mathbf{y}}$. In $\mathcal{R}_{1}$ and at time $t$, the deformed configuration occupies the bounded domain denoted by $\Omega(t) \subset \mathbb{R}^{3}$ with boundary $\partial \Omega(t)$. It is straightfoward to show that $\Omega(t)=\mathbf{X}_{t}(\widetilde{\Omega}(t))$ and $\partial \Omega(t)=\mathbf{X}_{t}(\partial \widetilde{\Omega}(t))$. Let $\Sigma(t), \Upsilon(t)$ and $\Gamma(t)$ be the parts of boundary $\partial \Omega(t)$ such that $\Sigma(t)=\mathbf{X}_{t}(\widetilde{\Sigma}(t)), \Upsilon(t)=\mathbf{X}_{t}(\widetilde{\Upsilon}(t))$ and $\Gamma(t)=\mathbf{X}_{t}(\widetilde{\Gamma}(t))$.

In $\mathcal{R}_{1}$ and at time $s$, let $\mathbf{X}\left(t, s, \mathbf{x}_{t}\right) \in \Omega(s)$ be the position of a particle which is located in $\mathcal{R}_{1}$ and at time $t>s$, at position $\mathbf{x}_{t} \in \Omega(t)$. Let $s \mapsto \mathbf{w}\left(t, s, \mathbf{x}_{t}\right)$ be the time history of the $\mathbb{R}^{3}$-valued displacement field of domain $\Omega(t)$, defined on ] $-\infty, t]$, such that

$$
\mathbf{w}\left(t, s, \mathbf{x}_{t}\right)=\mathbf{X}\left(t, s, \mathbf{x}_{t}\right)-\mathbf{x}_{t}
$$

It could be shown that [15], for all $\mathbf{x}_{t}$ in $\Upsilon(t)$,

$$
\mathbf{w}\left(t, s, \mathbf{x}_{t}\right)=0
$$

which means that reference frame $\mathcal{R}_{1}$ and all the material points belonging on $\widetilde{\Upsilon}(t)$ move with the same rigid body motion described by the rotation matrix $[Q(t)]$.

The natural configuration is defined as the structure at rest without any pre-stresses. In $\mathcal{R}_{0}$, the natural configuration occupies a bounded domain denoted as $\Omega_{0} \subset \mathbb{R}^{3}$ with boundary $\partial \Omega_{0}$. In $\mathcal{R}_{0}$ at time $t$, a particule located at $\mathbf{x}_{0} \in \Omega_{0}$ in the natural configuration will occupy the position $\widetilde{\mathbf{X}}_{0}\left(t, \mathbf{x}_{0}\right) \in \widetilde{\Omega}(t)$ in the deformed configuration. It should be noted that the natural configuration could be defined as the image of domain $\Omega_{0}$ by the mapping $\mathbf{x}_{0} \mapsto \mathcal{R}_{0}^{-1}\left(\mathbf{x}_{0}\right)$. We then introduce the rotating natural configuration as the image of domain $\Omega_{0}$ by the mapping $\mathbf{x}_{0} \mapsto \mathcal{R}_{1}^{-1}\left(\mathbf{x}_{0}\right)$. Note that, in $\mathcal{R}_{0}$, the rotating natural configuration occupies a domain $\widetilde{\Omega}_{0}(t)$ which is the image of $\Omega_{0}$ by the mapping $\mathbf{X}_{t}^{-1}$ justifying the rotating natural configuration terminology introduced. In $\mathcal{R}_{1}$ at time $t$, a particle located at position $\mathbf{x}_{0}$ in the rotating natural configuration will occupy the position denoted by $\mathbf{X}_{0}\left(t, \mathbf{x}_{0}\right) \in \Omega(t)$ in the deformed configuration. Let $\Sigma_{0}, \Upsilon_{0}$ and $\Gamma_{0}$ be the parts of boundary $\partial \Omega_{0}$ such that $\Sigma(t)=\mathbf{X}_{0}\left(t, \Sigma_{0}\right)$, $\Upsilon(t)=\mathbf{X}_{0}\left(t, \Upsilon_{0}\right)$ and $\Gamma(t)=\mathbf{X}_{0}\left(t, \Gamma_{0}\right)$. Considering Eq. (8), it could be noted that $\Upsilon(t)$ is a fixed part of boundary $\partial \Omega(t)$ denoted as $\Upsilon$. Consequently, the domain $\Omega_{0}$ should be chosen such that $\Upsilon_{0}=\Upsilon$. Let 
$\mathbf{u}_{0}\left(t, \mathbf{x}_{0}\right)$ be the $\mathbb{R}^{3}$-valued displacement field of the deformed configuration in $\mathcal{R}_{1}$ and at time $t$, defined on domain $\Omega_{0}$, such that

$$
\mathbf{u}_{0}\left(t, \mathbf{x}_{0}\right)=\mathbf{X}_{0}\left(t, \mathbf{x}_{0}\right)-\mathbf{x}_{0}, \quad \forall \mathbf{x}_{0} \in \Omega_{0}
$$

In general, $\mathbf{u}_{0}\left(0, \mathbf{x}_{0}\right)$ is not zero. Let $\mathbf{x}_{0} \mapsto \mathbf{G}_{0}\left(\tau, t, \mathbf{x}_{0}, \delta \mathbf{u}_{0}\right)$ be the $\mathbb{R}^{3}$-valued function defined on $\Sigma_{0}$ and such that for any function $\mathbf{y}_{0} \mapsto \delta \mathbf{u}_{0}\left(\mathbf{y}_{0}\right)$ from $\Sigma_{0}$ into $\mathbb{R}^{3}$, we have

$$
\mathbf{G}_{0}\left(\tau, t, \mathbf{x}_{0}, \delta \mathbf{u}_{0}\right) d S_{0}\left(\mathbf{x}_{0}\right)=[Q(t)] \widetilde{\mathbf{G}}\left(\tau, t, \widetilde{\mathbf{x}}_{t}, \delta \widetilde{\mathbf{w}}\right) d \widetilde{S}_{t}\left(\widetilde{\mathbf{x}}_{t}\right)
$$

where $d \widetilde{S}_{t}\left(\widetilde{\mathbf{x}}_{t}\right)$ is the surface element of the boundary $\partial \widetilde{\Omega}(t)$ and $d S_{0}\left(\mathbf{x}_{0}\right)$ is the surface element of the boundary $\partial \Omega_{0}$ with $\widetilde{\mathbf{x}}_{t}=\mathbf{X}_{0}\left(t, \mathbf{x}_{0}\right)$ for all $\mathbf{x}_{0}$ in $\Omega_{0}$ and where

$$
\delta \mathbf{w}(\widetilde{\mathbf{y}})=[Q(t)]\left\{\delta \mathbf{u}_{0}\left(\mathbf{y}_{0}\right)-\mathbf{u}_{0}\left(t, \mathbf{y}_{0}\right)\right\}+\{[Q(t-\tau)]-[Q(t)]\} \mathbf{X}_{0}\left(t, \mathbf{y}_{0}\right)
$$

with $\widetilde{\mathbf{y}}_{t}=\widetilde{\mathbf{X}}_{0}\left(t, \mathbf{y}_{0}\right)$ for all $\mathbf{y}_{0}$ in $\Upsilon_{0}$. With respect to the rotating natural configuration in $\mathcal{R}_{1}$, Eqs (2) to (6) of the three-dimensional rotating structure could be rewritten as (see for instance [15])

$$
\begin{aligned}
& \forall \mathbf{x}_{0} \in \Omega_{0}, \quad \operatorname{div}_{\mathbf{x}_{0}}\left(\mathbb{F}_{0}\left(t, \mathbf{x}_{0}\right) \mathbb{U}_{0}\left(t, \mathbf{x}_{0}\right)\right)+\rho_{0}\left(\mathbf{x}_{0}\right) \mathbf{f}_{0}\left(t, \mathbf{x}_{0}\right)=\rho_{0}\left(\mathbf{x}_{0}\right) \ddot{\mathbf{u}}_{0}\left(t, \mathbf{x}_{0}\right)+2 \rho_{0}\left(\mathbf{x}_{0}\right)[R] \dot{\mathbf{u}}_{0}\left(t, \mathbf{x}_{0}\right) \\
& +\rho_{0}\left(\mathbf{x}_{0}\right)\left\{[\dot{R}(t)]+[R(t)]^{2}\right\}\left\{\mathbf{x}_{0}+\mathbf{u}_{0}\left(t, \mathbf{x}_{0}\right)\right\}, \\
& \forall \mathbf{x}_{0} \in \Sigma_{0}, \quad \mathbb{F}_{0}\left(t, \mathbf{x}_{0}\right) \mathbb{U}_{0}\left(t, \mathbf{x}_{0}\right) \mathbf{n}_{0}\left(\mathbf{x}_{0}\right)=\mathbf{F}_{0}\left(t, \mathbf{x}_{0}\right), \\
& \forall \mathbf{x}_{0} \in \Upsilon_{0}, \quad \mathbf{u}_{0}\left(t, \mathbf{x}_{0}\right)=0, \\
& \forall \mathbf{x}_{0} \in \Gamma_{0}, \quad \mathbf{u}_{0}\left(t, \mathbf{x}_{0}\right)=\mathbf{u}_{0}^{\operatorname{dir}}\left(t, \mathbf{x}_{0}\right), \\
& \text { with } \quad \mathbf{F}_{0}\left(t, \mathbf{x}_{0}\right)=\int_{0}^{+\infty} \mathbf{G}_{0}\left(\tau, t, \mathbf{x}_{0},\left\{\mathbf{u}_{0}\left(t-\tau, \mathbf{y}_{0}\right)\right\}_{\mathbf{y}_{0} \in \Sigma_{0}}\right) d \tau \text {. }
\end{aligned}
$$

where, in $\mathcal{R}_{1}$ and at time $t$, the function $\mathbf{u}_{0}^{\operatorname{dir}}\left(t, \mathbf{x}_{0}\right)$ defined on $\Gamma_{0}$ with values in $\mathbb{R}^{3}$, is such that $\mathbf{u}_{0}^{d i r}\left(t, \mathbf{y}_{0}\right)=\mathbf{u}_{0}\left(0, \mathbf{y}_{0}\right)-[Q(s)]^{\mathrm{T}} \widetilde{\mathbf{w}}^{d i r}\left(0, t, \widetilde{\mathbf{y}}_{t}\right)+\left\{\left[Q(t)^{\mathrm{T}}-[Q(s)]^{\mathrm{T}}\right\} \widetilde{\mathbf{y}}_{t}\right.$ with $\widetilde{\mathbf{y}}_{t}=\widetilde{\mathbf{X}}_{0}\left(t, \mathbf{y}_{0}\right)$ for all $\mathbf{y}_{0}$ in $\Gamma_{0}$, where the unit outward normal vector to boundary $\partial \Omega_{0}$ is denoted by $\mathbf{n}_{0}\left(\mathbf{x}_{0}\right) \in \mathbb{R}^{3}$, where the tensorvalued function $\mathbb{F}_{0}\left(t, \mathbf{x}_{0}\right)=\partial_{\mathbf{x}_{0}} \mathbf{X}_{0}\left(t, \mathbf{x}_{0}\right)$, defined on $\Omega_{0}$, is the deformation gradient of the rotating natural configuration, where the mass density $\rho_{0}\left(\mathbf{x}_{0}\right)$ defined on domain $\Omega_{0}$ corresponds to the Lagrangian transport by $\widetilde{\mathbf{X}}_{0}$ of $\widetilde{\rho}\left(t, \widetilde{\mathbf{x}}_{t}\right)$, where the $\mathbb{R}^{3}$-valued function $\mathbf{f}_{0}=\left(\operatorname{det} \mathbb{F}_{0}\right)^{-1}[Q(t)]^{\mathrm{T}} \widetilde{\mathbf{f}} \circ \widetilde{\mathbf{X}}_{0}^{-1}$ is the density of the applied body forces per unit volume in the rotating natural configuration, where the $\mathbb{R}^{3}$-valued function $\mathbf{F}_{0}\left(t, \mathbf{x}_{0}\right)$, defined on $\Sigma_{0}$, is the density of the applied surface forces per unit area in the rotating natural configuration such that $\mathbf{F}_{0} d S_{0}=[Q(t)]^{\mathrm{T}}\left(\widetilde{\mathbf{F}} d \widetilde{S}_{t}\right) \circ \widetilde{\mathbf{X}}_{0}$ and where $\mathbb{U}_{0}=\left(\operatorname{det} \mathbb{F}_{0}\right) \mathbb{F}_{0}^{-1}\left\{\sigma \circ \mathbf{X}_{0}\right\} \mathbb{F}_{0}^{-\mathrm{T}}$ denotes the Piola-Kirchhoff stress tensor such that $\sigma\left(t, \mathbf{x}_{t}\right)=[Q(t)]^{\mathrm{T}} \widetilde{\widetilde{\sigma}}\left(t,[Q(t)] \mathbf{x}_{t}\right)[Q(t)]$ is the Cauchy stress tensor of the deformed configuration in $\mathcal{R}_{1}$. From Eq. (9), we deduce that $\mathbb{F}_{0}=[I]+\partial_{\mathbf{x}_{0}} \mathbf{u}_{0}$. It 
should be noted that if the rotational velocity and the axis of rotation are independant of $t$, then $[\dot{R}(t)]=0$ and $[R(t)]$ is a constant matrix denoted as $[R]$. With this assumption, Eq. (12) is rewritten as

$$
\begin{array}{r}
\operatorname{div}_{\mathbf{x}_{0}}\left(\mathbb{F}_{0}\left(t, \mathbf{x}_{0}\right) \mathbb{T}_{0}\left(t, \mathbf{x}_{0}\right)\right)+\rho_{0}\left(\mathbf{x}_{0}\right) \mathbf{f}_{0}\left(t, \mathbf{x}_{0}\right)=\rho_{0}\left(\mathbf{x}_{0}\right)[R]^{2}\left\{\mathbf{x}_{0}+\mathbf{u}_{0}\left(t, \mathbf{x}_{0}\right)\right\} \\
+2 \rho_{0}\left(\mathbf{x}_{0}\right)[R] \dot{\mathbf{u}}_{0}\left(t, \mathbf{x}_{0}\right) \\
+\rho_{0}\left(\mathbf{x}_{0}\right) \ddot{\mathbf{u}}_{0}\left(t, \mathbf{x}_{0}\right) .(17)
\end{array}
$$

\section{Nonlinear steady boundary value problem in the rotating frame}

The external loads applied to the structure and the displacement field $\mathbf{u}_{0}^{\text {dir }}$ can be decomposed:

$$
\begin{array}{cl}
\mathbf{f}_{0}\left(t, \mathbf{x}_{0}\right)=\mathbf{f}_{0}^{\text {stat }}\left(\mathbf{x}_{0}\right)+\mathbf{f}_{0}^{\text {dyn }}\left(t, \mathbf{x}_{0}\right), & \forall \mathbf{x}_{0} \in \Omega_{0}, \\
\mathbf{F}_{0}\left(t, \mathbf{x}_{0}\right)=\mathbf{F}_{0}^{\text {stat }}\left(\mathbf{x}_{0}\right)+\mathbf{F}_{0}^{\text {dyn }}\left(t, \mathbf{x}_{0}\right), & \forall \mathbf{x}_{0} \in \Sigma_{0}, \\
\mathbf{u}_{0}^{\text {dir }}\left(t, \mathbf{x}_{0}\right)=\mathbf{u}_{0}^{\text {stat }}\left(\mathbf{x}_{0}\right)+\mathbf{u}_{0}^{\text {dyn }}\left(t, \mathbf{x}_{0}\right), & \forall \mathbf{x}_{0} \in \Gamma_{0},
\end{array}
$$

where $t \mapsto \rho_{0}\left(\mathbf{x}_{0}\right) \mathbf{f}_{0}^{d y n}\left(t, \mathbf{x}_{0}\right)$ denotes the time-fluctuation part of body force $t \mapsto \rho_{0}\left(\mathbf{x}_{0}\right) \mathbf{f}_{0}\left(t, \mathbf{x}_{0}\right)$ around its steady part $\rho_{0}\left(\mathbf{x}_{0}\right) \mathbf{f}_{0}^{\text {stat }}\left(\mathbf{x}_{0}\right)$, where $t \mapsto \mathbf{F}_{0}^{d y n}\left(t, \mathbf{x}_{0}\right)$ denotes the time-fluctuation part of surface force $t \mapsto \mathbf{F}_{0}\left(t, \mathbf{x}_{0}\right)$ around its steady part $\mathbf{F}_{0}^{\text {stat }}\left(\mathbf{x}_{0}\right)$ and where $\mathbf{u}_{0}^{\text {dyn }}\left(t, \mathbf{x}_{0}\right)$ denotes the time-fluctuation part of applied displacement $\mathbf{u}_{0}^{\text {dir }}\left(t, \mathbf{x}_{0}\right)$ around its steady part $\mathbf{u}_{0}^{\text {stat }}\left(\mathbf{x}_{0}\right)$, these steady parts being defined below. If the structure is only subjected to steady parts $\rho_{0}\left(\mathbf{x}_{0}\right) \mathbf{f}_{0}^{\text {stat }}, \mathbf{F}_{0}^{\text {stat }}$ and $\mathbf{u}_{0}^{\text {stat }}$ of external loads, then the structure is in equilibrium in a steady configuration which occupies in $\mathcal{R}_{1}$ a bounded domain denoted as $\Omega_{r}$ with boundary $\partial \Omega_{r}$. In $\mathcal{R}_{1}$, a particule which is located at position $\mathbf{x}_{0} \in \Omega_{0}$ in the rotating natural configuration will occupy the position $\mathbf{X}_{0}^{r e f}\left(\mathbf{x}_{0}\right) \in \Omega_{r}$ in the steady configuration. Let $\Sigma_{r}, \Upsilon_{r}$ and $\Gamma_{r}$ be the parts of boundary $\partial \Omega_{r}$ such that $\Sigma_{r}=\mathbf{X}_{0}^{r e f}\left(\Sigma_{0}\right), \Upsilon_{r}=\mathbf{X}_{0}^{r e f}\left(\Upsilon_{0}\right)$ and $\Gamma_{r}=\mathbf{X}_{0}^{r e f}\left(\Gamma_{0}\right)$. Let $\mathbf{u}_{0}^{r e f}\left(\mathbf{x}_{0}\right)$ be the $\mathbb{R}^{3}$-valued displacement field of the steady configuration in $\mathcal{R}_{1}$, defined from $\Omega_{0}$, such that

$$
\mathbf{u}_{0}^{r e f}\left(\mathbf{x}_{0}\right)=\mathbf{X}_{0}^{r e f}\left(\mathbf{x}_{0}\right)-\mathbf{x}_{0}, \quad \forall \mathbf{x}_{0} \in \Omega_{0}
$$

Therefore, $\mathbb{F}_{0}^{r e f}=\partial_{\mathbf{x}_{0}} \mathbf{X}_{0}^{\text {ref }}$ is the deformation gradient of the steady configuration defined on domain $\Omega_{0}$. Using Eq. (21) we deduce that $\mathbb{F}_{0}^{r e f}=[I]+\partial_{\mathbf{x}_{0}} \mathbf{u}_{0}^{\text {ref }}$. The Cauchy stress tensor of the steady configuration is denoted as $\sigma_{r}\left(\mathbf{x}_{r}\right)$ and its Piola-Kirchhoff transport [11] by $\mathbf{X}_{0}^{r e f}$ is the tensor-valued function $\mathbb{T}_{0}^{r e f}=\left(\operatorname{det} \mathbb{F}_{0}^{r e f}\right)\left\{\mathbb{F}_{0}^{r e f}\right\}^{-1}\left\{\sigma_{r} \circ \mathbf{X}_{0}^{r e f}\right\}\left\{\mathbb{F}_{0}^{r e f}\right\}^{-\mathrm{T}}$ defined on $\Omega_{0}$. If the functions $\mathbf{f}_{0}\left(t, \mathbf{x}_{0}\right)$, $\mathbf{u}_{0}^{\operatorname{dir}}\left(t, \mathbf{x}_{0}\right)$ and $\mathbf{G}_{0}\left(\tau, t, \mathbf{x}_{0}, \delta \mathbf{u}_{0}\right)$ are $T$-periodic in $t$ (which is consistent with the constant rotational 
velocity assumption), then $\mathbf{f}_{0}^{\text {stat }}\left(\mathbf{x}_{0}\right), \mathbf{F}_{0}^{\text {stat }}\left(\mathbf{x}_{0}\right)$ and $\mathbf{u}_{0}^{\text {stat }}\left(\mathbf{x}_{0}\right)$ are defined by

$$
\begin{gathered}
\mathbf{f}_{0}^{\text {stat }}\left(\mathbf{x}_{0}\right)=\frac{1}{T} \int_{0}^{T} \mathbf{f}_{0}\left(t, \mathbf{x}_{0}\right) d t, \quad \forall \mathbf{x}_{0} \in \Omega_{0}, \\
\mathbf{F}_{0}^{\text {stat }}\left(\mathbf{x}_{0}\right)=\frac{1}{T} \int_{0}^{+\infty} d \tau \int_{0}^{T} \mathbf{G}_{0}\left(\tau, t, \mathbf{x}_{0},\left\{\mathbf{u}_{0}^{r e f}\left(\mathbf{y}_{0}\right)\right\} \mathbf{y}_{0} \in \Sigma_{0}\right) d t, \quad \forall \mathbf{x}_{0} \in \Upsilon_{0}, \\
\mathbf{u}_{0}^{\text {stat }}\left(\mathbf{x}_{0}\right)=\frac{1}{T} \int_{0}^{T} \mathbf{u}_{0}^{\text {dir }}\left(t, \mathbf{x}_{0}\right) d t, \quad \forall \mathbf{x}_{0} \in \Sigma_{0} .
\end{gathered}
$$

In $\mathcal{R}_{1}$, the nonlinear steady boundary value problem is deduced from the nonlinear dynamical boundary value problem by replacing $\left\{\mathbf{f}_{0}, \mathbf{F}_{0}, \mathbf{u}_{0}^{\text {dir }}, \mathbf{u}_{0}, \mathbb{F}_{0}, \mathbb{U}_{0}\right\}$ with $\left\{\mathbf{f}_{0}^{\text {stat }}, \mathbf{F}_{0}^{\text {stat }}, \mathbf{u}_{0}^{\text {stat }}, \mathbf{u}_{0}^{\text {ref }}, \mathbb{F}_{0}^{\text {ref }}, \mathbb{U}_{0}^{\text {ref }}\right\}$ in Eqs. (12) to (15) and (17), and is written as (see for instance [15])

$$
\begin{aligned}
& \forall \mathbf{x}_{0} \in \Omega_{0}, \operatorname{div}_{\mathbf{x}_{0}}\left(\mathbb{F}_{0}^{r e f}\left(\mathbf{x}_{0}\right) \mathbb{U}_{0}^{r e f}\left(\mathbf{x}_{0}\right)\right)+\rho_{0}\left(\mathbf{x}_{0}\right) \mathbf{f}_{0}^{\text {stat }}\left(\mathbf{x}_{0}\right)=\rho_{0}\left(\mathbf{x}_{0}\right)[R]^{2}\left(\mathbf{x}_{0}+\mathbf{u}_{0}^{\text {ref }}\left(\mathbf{x}_{0}\right)\right) \\
& \forall \mathbf{x}_{0} \in \Sigma_{0}, \mathbb{F}_{0}^{r e f}\left(\mathbf{x}_{0}\right) \mathbb{T}_{0}^{r e f}\left(\mathbf{x}_{0}\right) \mathbf{n}_{0}\left(\mathbf{x}_{0}\right)=\mathbf{F}_{0}^{\text {stat }}\left(\mathbf{x}_{0}\right), \\
& \forall \mathbf{x}_{0} \in \Upsilon_{0}, \mathbf{u}_{0}^{r e f}\left(\mathbf{x}_{0}\right)=0 \\
& \forall \mathbf{x}_{0} \in \Gamma_{0}, \mathbf{u}_{0}^{r e f}\left(\mathbf{x}_{0}\right)=\mathbf{u}_{0}^{\text {stat }}\left(\mathbf{x}_{0}\right) .
\end{aligned}
$$

\section{Nonlinear dynamic boundary value problem in the rotating frame with respect to the reference configuration}

Below, we refer the steady configuration as the reference configuration of the nonlinear dynamical boundary value problem. Consequently, Eqs. (12) to (17), (25) and (26) have to be transported onto the domain $\Omega_{r}$. In $\mathcal{R}_{1}$ and at time $t$, a particule located at position $\mathbf{x}_{r}$ in the reference configuration will occupy the position denoted as $\mathbf{X}_{r}\left(t, \mathbf{x}_{r}\right) \in \Omega(t)$ in the deformed configuration. Let $\mathbf{u}_{r}\left(t, \mathbf{x}_{r}\right)$ be the $\mathbb{R}^{3}$-valued displacement field of the deformed configuration, defined on $\Omega_{r}$, such that

$$
\mathbf{u}_{r}\left(t, \mathbf{x}_{r}\right)=\mathbf{X}_{r}\left(t, \mathbf{x}_{r}\right)-\mathbf{x}_{r}
$$

Let $\mathbf{x}_{r} \mapsto \mathbf{G}_{r}\left(\tau, t, \mathbf{x}_{r}, \delta \mathbf{u}_{r}\right)$ be the mapping from $\Sigma_{r}$ into $\mathbb{R}^{3}$ such that, with $\mathbf{x}_{0}=\left\{\mathbf{X}_{0}^{r e f}\right\}^{-1}\left(\mathbf{x}_{r}\right)$ in $\Sigma_{0}$ and for any function $\delta \mathbf{u}_{0}$ from $\Sigma_{0}$ into $\mathbb{R}^{3}$, we have

$$
\mathbf{G}_{r}\left(\tau, t, \mathbf{x}_{r}, \delta \mathbf{u}_{r}\right) d S_{r}\left(\mathbf{x}_{r}\right)=\mathbf{G}_{0}\left(\tau, t, \mathbf{x}_{0}, \delta \mathbf{u}_{r} \circ \mathbf{X}_{0}^{r e f}+\mathbf{u}_{0}^{r e f}\right) d S_{0}\left(\mathbf{x}_{0}\right)
$$

where $\left(\delta \mathbf{u}_{r} \circ \mathbf{X}_{0}^{r e f}\right)\left(\mathbf{x}_{0}\right)$ means $\left.\delta \mathbf{u}_{r}\left(\mathbf{X}_{0}^{r e f}\right)\left(\mathbf{x}_{0}\right)\right)$ and where $d S_{r}\left(\mathbf{x}_{r}\right)$ is the surface element of boundary $\partial \Omega_{r}$. Equations (13) to (17) can be rewritten as (for instance, see [15])

$$
\begin{aligned}
\forall \mathbf{x}_{r} \in \Omega_{r}, \quad \operatorname{div}_{\mathbf{x}_{r}}\left(\mathbb{F}_{r}\left(t, \mathbf{x}_{0}\right) \mathbb{W}_{r}\left(t, \mathbf{x}_{r}\right)\right) & +\rho_{r}\left(\mathbf{x}_{r}\right) \mathbf{f}_{r}\left(t, \mathbf{x}_{r}\right)=\rho_{r}\left(\mathbf{x}_{r}\right) \ddot{\mathbf{u}}_{r}\left(t, \mathbf{x}_{r}\right)+2 \rho_{r}\left(\mathbf{x}_{0}\right)[R] \dot{\mathbf{u}}_{r}\left(t, \mathbf{x}_{r}\right) \\
& +\rho_{r}\left(\mathbf{x}_{r}\right)[R(t)]^{2}\left\{\mathbf{x}_{0}+\mathbf{u}_{0}\left(t, \mathbf{x}_{0}\right)\right\}
\end{aligned}
$$


$\forall \mathbf{x}_{r} \in \Sigma_{r}, \quad \mathbb{F}_{r}\left(t, \mathbf{x}_{r}\right) \mathbb{U}_{r}\left(t, \mathbf{x}_{r}\right) \mathbf{n}_{r}\left(\mathbf{x}_{r}\right)=\mathbf{F}_{r}\left(t, \mathbf{x}_{r}\right)$,

$\forall \mathbf{x}_{r} \in \Upsilon_{r}, \quad \mathbf{u}_{r}\left(t, \mathbf{x}_{r}\right)=0$,

$\forall \mathbf{x}_{r} \in \Gamma_{r}, \quad \mathbf{u}_{r}\left(t, \mathbf{x}_{r}\right)=\mathbf{u}_{r}^{\operatorname{dir}}\left(t, \mathbf{x}_{r}\right)$,

with $\quad \mathbf{F}_{r}\left(t, \mathbf{x}_{r}\right)=\int_{0}^{+\infty} \mathbf{G}_{r}\left(\tau, t, \mathbf{x}_{r},\left\{\mathbf{u}_{r}\left(t-\tau, \mathbf{y}_{r}\right)\right\}_{\mathbf{y}_{r} \in \Sigma_{r}}\right) d \tau$.

where, in $\mathcal{R}_{1}$ and at time $t$, the function $\mathbf{u}_{r}^{\operatorname{dir}}\left(t, \mathbf{x}_{r}\right)$ defined on $\Gamma_{r}$ with values in $\mathbb{R}^{3}$, is such that $\mathbf{u}_{r}^{\text {dir }}\left(t, \mathbf{x}_{r}\right)=\mathbf{u}_{0}^{\text {dir }}\left(t,\left\{\mathbf{X}_{0}^{\text {ref }}\right\}^{-1}\left(\mathbf{x}_{r}\right)\right)-\mathbf{u}_{0}^{\text {stat }}\left(\left\{\mathbf{X}_{0}^{\text {ref }}\right\}^{-1}\left(\mathbf{x}_{r}\right)\right)$, the unit outward normal vector to the boundary $\partial \Omega_{r}$ is denoted as $\mathbf{n}_{r}\left(\mathbf{x}_{r}\right) \in \mathbb{R}^{3}$, the tensor-valued function $\mathbb{F}_{r}\left(t, \mathbf{x}_{r}\right)=\partial_{\mathbf{x}_{r}} \mathbf{X}_{r}\left(t, \mathbf{x}_{r}\right)$, defined on $\Omega_{r}$, is the deformation gradient of the rotating natural configuration, the mass density $\rho_{r}\left(\mathbf{x}_{r}\right)$ defined on domain $\Omega_{r}$ corresponds to the Lagrangian transport by $\mathbf{X}_{0}^{r e f}$ of $\rho_{0}\left(\mathbf{x}_{0}\right)$, the $\mathbb{R}^{3}$-valued function $\mathbf{f}_{r}\left(t, \mathbf{x}_{r}\right)$ is the density of the applied body forces per unit volume in the rotating natural configuration such that $\rho_{r}\left(\mathbf{x}_{r}\right) \mathbf{f}_{r}\left(t, \mathbf{x}_{r}\right) d \mathbf{x}_{r}=\rho_{0}\left(\mathbf{x}_{0}\right) \mathbf{f}_{0}\left(t, \mathbf{x}_{0}\right) d \mathbf{x}_{0}$, the $\mathbb{R}^{3}$-valued function $\mathbf{F}_{0}\left(t, \mathbf{x}_{0}\right)$, defined on $\Sigma_{0}$, is the density of the applied surface forces per unit area in the rotating natural configuration such that $\mathbf{F}_{0} d S_{0}=\left(\mathbf{F}_{r} d S_{r}\right) \circ \mathbf{X}_{0}^{r e f}$ and $\mathbb{U}_{r}=\left(\operatorname{det} \mathbb{F}_{r}\right) \mathbb{F}_{r}^{-1}\left\{\sigma \circ \mathbf{X}_{0}^{r e f}\right\} \mathbb{F}_{r}^{-\mathrm{T}}$ denotes the Piola-Kirchhoff stress tensor. From Eq. (29), we deduce that $\mathbb{F}_{r}=[I]+\partial_{\mathbf{x}_{0}} \mathbf{u}_{r}$.

In $\mathcal{R}_{1}$ and at time $t$, the nonlinear dynamical boundary value problem of the rotating structures with respect to the reference configuration (the steady configuration) is given by Eqs. (31) to (35). Consequently, the transport of Eq. (25) on domain $\Omega_{r}$ and the transport of Eq. (26) on $\Sigma_{r}$ give

$$
\begin{gathered}
\operatorname{div}_{\mathbf{x}_{r}} \sigma_{r}\left(\mathbf{x}_{r}\right)+\rho_{r}\left(\mathbf{x}_{r}\right) \mathbf{f}_{r}^{\text {stat }}\left(\mathbf{x}_{r}\right)=\rho_{r}\left(\mathbf{x}_{r}\right)[R]^{2} \mathbf{x}_{r}, \quad \forall \mathbf{x}_{r} \in \Omega_{r}, \\
\sigma_{r}\left(\mathbf{x}_{r}\right) \mathbf{n}_{r}=\mathbf{F}_{r}^{\text {stat }}\left(\mathbf{x}_{r}\right), \quad \forall \mathbf{x}_{r} \in \Sigma_{r},
\end{gathered}
$$

where $\rho_{r}\left(\mathbf{x}_{r}\right) \mathbf{f}_{r}^{\text {stat }}\left(\mathbf{x}_{r}\right)$ defined on $\Omega_{r}$, is the external body forces applied to the reference configuration (the steady configuration) and corresponds to the Lagrangian transport by $\mathbf{X}_{0}^{\text {ref }}$ of external body forces $\rho_{0}\left(\mathbf{x}_{0}\right) \mathbf{f}_{0}^{\text {stat }}\left(\mathbf{x}_{0}\right)$ defined on domain $\Omega_{0}$ and where $\mathbf{F}_{r}^{\text {stat }}\left(\mathbf{x}_{r}\right)$ is the external surface forces applied to boundary $\Sigma_{r}$ corresponding to the Lagrangian transport by $\mathbf{X}_{0}^{\text {ref }}$ of surface forces $\mathbf{F}_{0}^{\text {stat }}\left(\mathbf{x}_{0}\right)$ defined $\Sigma_{0}$. It should be noted that

$$
\mathbf{F}_{r}^{\text {stat }}\left(\mathbf{x}_{r}\right)=\frac{1}{T} \int_{0}^{+\infty} d \tau \int_{0}^{T} \mathbf{G}_{r}\left(\tau, t, \mathbf{x}_{r}, 0\right) d t, \quad \forall \mathbf{x}_{r} \in \Sigma_{r}
$$




\section{Viscoelasticity in finite displacement.}

In this paper, we consider viscoelastic materials and we refer the reader to the theory of linear viscoelasticity in finite displacement developed by B.D. Coleman and W. Noll [13]. In order to deduce a total Lagrangian formulation of the constitutive equation, it is necessary to present a short review of this theory. It is assumed that $\operatorname{det} \widetilde{\mathbb{F}}_{0}\left(t, \mathbf{x}_{0}\right)>0$, where $\widetilde{\mathbb{F}}_{0}\left(t, \mathbf{x}_{0}\right)=\partial_{\mathbf{x}_{0}} \widetilde{\mathbf{x}}_{0}\left(t, \mathbf{x}_{0}\right)$ is the deformation gradient of the natural configuration in $\mathcal{R}_{0}$, at time $t$. For $s \leq t$, let $\widetilde{\mathbb{E}}\left(t, s, \widetilde{\mathbf{x}}_{t}\right)=\frac{1}{2}\left(\widetilde{\mathbb{F}}^{T} \widetilde{\mathbb{F}}-[I]\right)$ be the Green-Lagrange strain tensor defined on domain $\widetilde{\Omega}(t)$ in which $\widetilde{\mathbb{F}}\left(t, s, \widetilde{\mathbf{x}}_{t}\right)=\partial_{\tilde{\mathbf{x}}_{t}} \widetilde{\mathbf{X}}\left(t, s, \widetilde{\mathbf{x}}_{t}\right)$. From Eq. (2), we deduce that $\widetilde{\mathbb{F}}=[I]+\partial_{\tilde{\mathbf{x}}_{t}} \widetilde{\mathbf{w}}$. The theory of linear viscoelaticity in finite displacement states that Cauchy stress tensor $\widetilde{\sigma}\left(t, \widetilde{\mathbf{x}}_{t}\right)$ is such that

$$
\begin{aligned}
\widetilde{\mathbb{P}}_{0}\left(t, \mathbf{x}_{0}\right)^{-1} \widetilde{\widetilde{\sigma}}\left(t, \widetilde{\mathbf{X}}_{0}\left(t, \mathbf{x}_{0}\right)\right) \widetilde{\mathbb{P}}_{0}\left(t, \mathbf{x}_{0}\right)=\mathbb{h}_{0}\left(\mathbf{x}_{0}, \widetilde{\mathbb{E}}_{0}\left(t, \mathbf{x}_{0}\right)\right) \\
\quad+\int_{0}^{+\infty} \Gamma_{0}\left(\tau, \mathbf{x}_{0}, \widetilde{\mathbb{E}}_{0}\left(t, \mathbf{x}_{0}\right)\right):\left\{\widetilde{\mathbb{P}}_{0}\left(t, \mathbf{x}_{0}\right)^{\mathrm{T}} \widetilde{\mathbb{E}}\left(t, t-\tau, \widetilde{\mathbf{X}}_{0}\left(t, \mathbf{x}_{0}\right)\right) \widetilde{\mathbb{P}}_{0}\left(t, \mathbf{x}_{0}\right)\right\} d \tau,
\end{aligned}
$$

where $\widetilde{\mathbb{E}}_{0}=\frac{1}{2}\left\{\widetilde{\mathbb{F}}_{0}^{\mathrm{T}} \widetilde{\mathbb{F}}_{0}-[I]\right\}$ is the Green-Lagrange strain tensor of the deformed configuration in $\mathcal{R}_{0}$ with respect to domain $\Omega_{0}$ and where the orthogonal tensor $\widetilde{\mathbb{P}}_{0}\left(t, \mathbf{x}_{0}\right)$ is constructed using the polar decomposition of positive-definite tensor $\widetilde{\mathbb{F}}_{0}\left(t, \mathbf{x}_{0}\right)$ and is written as $\widetilde{\mathbb{P}}_{0}=\widetilde{\mathbb{F}}_{0}\left\{\widetilde{\mathbb{F}}_{0}^{\mathrm{T}} \widetilde{\mathbb{F}}_{0}\right\}^{-\frac{1}{2}}$.

In Eq. (39), $\mathbf{x}_{0} \mapsto \mathfrak{h}_{0}\left(\mathbf{x}_{0}, \widetilde{\mathbb{E}}_{0}\left(t, \mathbf{x}_{0}\right)\right)$ is a function from $\Omega_{0}$ into the set of all symmetric second-order tensors and $t \mapsto \Gamma_{0}\left(t, \mathbf{x}_{0}, \mathbb{V}\right)$ is a function from $\mathbb{R}^{+}$into the set of all symmetric fourth-order tensors. Futhermore, for all symmetric second-order tensors $\mathbb{V}$, for all $\mathbf{x}_{0}$ in $\Omega_{0}$ and for all $i, j, k, h$ fixed in $\{1,2,3\}$, functions $t \mapsto\left\{\Gamma_{0}\left(t, \mathbf{x}_{0}, \mathbb{V}\right)\right\}_{i j k h}$ belongs to $L^{2}\left(\mathbb{R}^{+}, \gamma^{-2} d \tau\right)$ where $\gamma$ is a positive real-value function defined on $\mathbb{R}^{+}$such that, for a given positive integer $r, \lim _{\tau \mapsto+\infty} \tau^{r} \gamma(\tau)=0$.

In a first step, the constitutive equation (39) could be rewritten in rotating reference frame $\mathcal{R}_{1}$ in which a Lagrangian formulation on domain $\Omega_{0}$ for the linear viscoelasticity in finite displacement theory could be deduced. In a last step, this Lagrangian formulation is transported onto domain $\Omega_{r}$.

Thus, Eq. (39) could be rewritten as (see for instance [15])

$$
\begin{aligned}
\mathbb{\amalg}_{0}\left(t, \mathbf{x}_{0}\right)=\mathbb{H}_{0}\left(\mathbf{x}_{0}, \mathbb{E}_{0}\left(t, \mathbf{x}_{0}\right)\right) & +\Phi_{0}\left(0, \mathbf{x}_{0}, \mathbb{E}_{0}\left(t, \mathbf{x}_{0}\right)\right): \mathbb{E}_{0}\left(t, \mathbf{x}_{0}\right) \\
& +\int_{0}^{+\infty} \dot{\Phi}_{0}\left(\tau, \mathbf{x}_{0}, \mathbb{E}_{0}\left(t, \mathbf{x}_{0}\right)\right): \mathbb{E}_{0}\left(t-\tau, \mathbf{x}_{0}\right) d \tau,
\end{aligned}
$$

in which $\mathbb{E}_{0}=\frac{1}{2}\left\{\mathbb{F}_{0}^{\mathrm{T}} \mathbb{F}_{0}-[I]\right\}$ is the Green-Lagrange strain tensor of the deformed configuration in $\mathcal{R}_{0}$ with respect to domain $\Omega_{0}$, and where the derivative of function $\Phi_{0}\left(\tau, \mathbf{x}_{0}, \mathbb{V}\right)$ with respect to $\tau$ is written 


$$
\begin{aligned}
\left\{\Phi_{0}\left(s, \mathbf{x}_{0}, \mathbb{V}\right)\right\}_{i j k h} & =\operatorname{det}(2 \mathbb{V}+[I])^{\frac{1}{2}} \sum_{a, b, p, q=1}^{3}\left\{(2 \mathbb{V}+[I])^{-\frac{1}{2}}\right\}_{a i}\left\{(2 \mathbb{V}+[I])^{-\frac{1}{2}}\right\}_{b j} \\
& \times\left\{(2 \mathbb{V}+[I])^{-\frac{1}{2}}\right\}_{p k}\left\{(2 \mathbb{V}+[I])^{-\frac{1}{2}}\right\}_{q h}\left\{\Gamma_{0}\left(s, \mathbf{x}_{0}, \mathbb{V}\right)\right\}_{a b p q},
\end{aligned}
$$

where $\mathbb{H}_{0}\left(\mathbf{x}_{0}, \mathbb{V}\right)$ is such that, for all symmetric second-order tensors $\mathbb{V}$,

$$
\uplus_{0}\left(\mathbf{x}_{0}, \mathbb{V}\right)=\operatorname{det}(2 \mathbb{V}+[I])^{\frac{1}{2}}(2 \mathbb{V}+[I])^{-\frac{1}{2}} \bigcap_{0}\left(\mathbf{x}_{0}, \mathbb{V}\right)(2 \mathbb{V}+[I])^{-\frac{1}{2}} .
$$

In addition, we have $\lim _{\tau \rightarrow+\infty} \Phi_{0}\left(\tau, \mathbf{x}_{0}, \mathbb{V}\right)=0$. If $\mathbb{E}_{0}\left(\tau, \mathbf{x}_{0}\right)$ is a bounded function in $\tau$ and if $\tau \mapsto \Phi_{0}\left(\tau, \mathbf{x}_{0}, \mathbb{V}\right)$ belongs to $L^{1}\left(\mathbb{R}^{+}, d \tau\right)$ then $\mathbb{U}_{0}\left(t, \mathbf{x}_{0}\right)$ exists. With these assumptions and because $\left.\tau \mapsto \Gamma_{0}\left(\tau, \mathbf{x}_{0}, \mathbb{V}\right)\right)$ belongs to $L^{2}\left(\mathbb{R}^{+}, \gamma^{-2} d \tau\right)$, we deduce that function $\tau \mapsto \Phi_{0}\left(\tau, \mathbf{x}_{0}, \mathbb{V}\right)$ belongs to $L^{2}\left(\mathbb{R}^{+}, \gamma^{-2} d \tau\right) \cap L^{1}\left(\mathbb{R}^{+}, d \tau\right)$.

In this paper, only finite displacements are taken into account and material nonlinearities are not considered. Consequently, substituting $\mathbb{H}_{0}\left(\mathbf{x}_{0}, \mathbb{E}_{0}\left(t, \mathbf{x}_{0}\right)\right)=\mathbb{A}_{0}\left(\mathbf{x}_{0}\right): \mathbb{E}_{0}\left(t, \mathbf{x}_{0}\right)$ into Eq. (40) and introducing the $\mathbb{G}_{0}\left(\tau, \mathbf{x}_{0}\right)=\Phi_{0}\left(\tau, \mathbf{x}_{0}, 0\right)+\mathbb{A}_{0}\left(\mathbf{x}_{0}\right)$ yield

$$
\mathbb{U T}_{0}\left(t, \mathbf{x}_{0}\right)=\mathbb{G}_{0}\left(0, \mathbf{x}_{0}\right): \mathbb{E}_{0}\left(t, \mathbf{x}_{0}\right)+\int_{0}^{+\infty} \dot{\mathbb{G}}_{0}\left(\tau, \mathbf{x}_{0}\right): \mathbb{E}_{0}\left(t-\tau, \mathbf{x}_{0}\right) d \tau .
$$

It can be proven that

$$
\left\{\mathbb{G}_{0}\left(t, \mathbf{x}_{0}\right)\right\}_{i j k h}=\left\{\mathbb{G}_{0}\left(t, \mathbf{x}_{0}\right)\right\}_{k h i j}=\left\{\mathbb{G}_{0}\left(t, \mathbf{x}_{0}\right)\right\}_{i j h k}=\left\{\mathbb{G}_{0}\left(t, \mathbf{x}_{0}\right)\right\}_{j i k h},
$$

and that there exists $c>0$ such that, for all symmetric second-order tensors $\mathfrak{X}$,

$$
\left\{\mathbb{G}_{0}\left(0, \mathbf{x}_{0}\right)\right\}_{i j k h}\{\mathbb{X}\}_{k h}\{\mathbb{X}\}_{i j} \geq c\{\mathbb{X}\}_{i j}\{\mathbb{X}\}_{i j}
$$

It should be noted that the linearization of Eq. (43) with respect to $\mathbf{u}_{0}$ yields the usual linear viscoelasticity theory $[20,31,43]$

$$
\sigma\left(t, \mathbf{X}_{0}\left(t, \mathbf{x}_{0}\right)\right)=\mathbb{G}_{0}\left(0, \mathbf{x}_{0}\right): \mathbb{E}_{0}\left(t, \mathbf{x}_{0}\right)+\int_{0}^{+\infty} \dot{\mathbb{G}}_{0}\left(\tau, \mathbf{x}_{0}\right): \mathbb{E}_{0}\left(t-\tau, \mathbf{x}_{0}\right) d \tau
$$

where $\mathbb{E}_{0}=\frac{1}{2}\left\{\partial_{\mathbf{x}_{0}} \mathbf{u}_{0}+\partial_{\mathbf{x}_{0}} \mathbf{u}_{0}^{\mathrm{T}}\right\}$ denotes the linearized strain tensor. Substituting $\mathbb{G}_{0}\left(\tau, \mathbf{x}_{0}\right) d \tau=$ $\mathbb{A}_{0}\left(\mathbf{x}_{0}\right) 1_{\mathbb{R}^{+}}(\tau) d \tau+\mathbb{B}_{0}\left(\mathbf{x}_{0}\right) \delta_{0}(\tau)$ into the right-hand side of Eq. (43) yields the constitutive equation of instantaneous viscoelasticity in finite displacement which is written as

$$
\mathbb{U T}_{0}\left(t, \mathbf{x}_{0}\right)=\mathbb{A}_{0}\left(\mathbf{x}_{0}\right): \mathbb{E}_{0}\left(t, \mathbf{x}_{0}\right)+\mathbb{B}_{0}\left(0, \mathbf{x}_{0}\right): \dot{\mathbb{E}}_{0}\left(t, \mathbf{x}_{0}\right) .
$$


Let $\mathbb{E}_{0}^{r e f}=\frac{1}{2}\left(\mathbb{F}_{0}^{r e f^{\mathrm{T}}} \mathbb{F}_{0}^{r e f}-[I]\right)$ be the Green-Lagrange strain tensor of the reference configuration defined on $\Omega_{0}$. Substituting $\mathbb{E}_{0}\left(t, \mathbf{x}_{0}\right)=\mathbb{E}_{0}^{\text {ref }}\left(\mathbf{x}_{0}\right)$ into Eq. (43) yields

$$
\mathbb{W}_{0}^{r e f}\left(\mathbf{x}_{0}\right)=\mathbb{A}_{0}\left(\mathbf{x}_{0}\right): \mathbb{E}_{0}^{r e f}\left(\mathbf{x}_{0}\right), \quad \forall \mathbf{x}_{0} \in \Omega_{0}
$$

In section 4, we introduced $\mathbb{T}_{0}^{r e f}$ as the Piola-Kirchhoff transport by $\mathbf{X}_{0}^{r e f}$ of tensor-valued function $\sigma_{r}$. Consequently, we deduce from Eq. (48)

$$
\sigma_{r}\left(\mathbf{x}_{r}\right)=\frac{1}{\operatorname{det} \mathbb{F}_{0}^{r e f}\left(\mathbf{x}_{0}\right)} \mathbb{F}_{0}^{r e f}\left(\mathbf{x}_{0}\right)\left\{\mathbb{A}_{0}\left(\mathbf{x}_{0}\right): \mathbb{E}_{0}^{r e f}\left(\mathbf{x}_{0}\right)\right\} \mathbb{F}_{0}^{r e f}\left(\mathbf{x}_{0}\right)^{\mathrm{T}},
$$

with $\mathbf{x}_{0}=\left\{\mathbf{X}_{0}^{r e f}\right\}^{-1}\left(\mathbf{x}_{r}\right)$. Let $\mathbb{E}_{r}\left(t, \mathbf{x}_{r}\right)=\frac{1}{2}\left(\mathbb{F}_{r}^{\mathrm{T}} \mathbb{F}_{r}-[I]\right)$ be the Green-Lagrange strain tensor of the deformed configuration defined on $\Omega_{r}$. In section 5 , we have introduced $\mathbb{U}_{r}$ as the Piola-Kirchhoff transport by $\mathbf{X}_{r}$ of Cauchy stress tensor $\sigma$. In section 3, $\mathbb{\pi}_{0}$ is also defined as the Piola-Kirchhoff transport by $\mathbf{X}_{0}$ of Cauchy stress tensor $\sigma$. Therefore, it could be proved [15] that $\mathbb{\pi}_{r}$ is also the Piola-Kirchhoff transport by $\mathbf{X}_{o}^{r e f}$ of tensor-valued function $\mathbb{U}_{0}$. Consequently, Eq. (47) could be rewritten as

$$
\mathbb{U}_{r}\left(t, \mathbf{x}_{r}\right)=\sigma_{r}\left(\mathbf{x}_{r}\right)+\mathbb{G}_{r}\left(0, \mathbf{x}_{r}\right): \mathbb{E}_{r}\left(t, \mathbf{x}_{r}\right)+\int_{0}^{+\infty} \dot{\mathbb{G}}_{r}\left(\tau, \mathbf{x}_{r}\right): \mathbb{E}_{r}\left(t-\tau, \mathbf{x}_{r}\right) d \tau,
$$

where $\mathbb{G}_{r}\left(\tau, \mathbf{x}_{r}\right)$ is such that

$$
\left\{\mathbb{G}_{r}\left(\tau, \mathbf{x}_{r}\right)\right\}_{a b p q}=\frac{\left\{\mathbb{G}_{0}\left(\tau, \mathbf{x}_{0}\right)\right\}_{k l m n}}{\operatorname{det} \mathbb{F}_{0}^{r e f}\left(\mathbf{x}_{0}\right)}\left\{\mathbb{F}_{0}^{r e f}\left(\mathbf{x}_{0}\right)\right\}_{a k}\left\{\mathbb{F}_{0}^{r e f}\left(\mathbf{x}_{0}\right)\right\}_{b l}\left\{\mathbb{F}_{0}^{r e f}\left(\mathbf{x}_{0}\right)\right\}_{p m}\left\{\mathbb{F}_{0}^{r e f}\left(\mathbf{x}_{0}\right)\right\}_{q n}
$$

in which $\mathbf{x}_{0}=\left\{\mathbf{X}_{0}^{r e f}\right\}^{-1}\left(\mathbf{x}_{r}\right)$. It is easy to prove that, for any $\{a, b, p, q\}$ fixed in $\{1,2,3\}$, functions $\tau \mapsto\left\{\mathbb{G}_{r}\left(\tau, \mathbf{x}_{r}\right)\right\}_{\text {abpq }}$ belong to $L^{2}\left(\mathbb{R}^{+}, \gamma^{-2} d \tau\right) \cap L^{1}\left(\mathbb{R}^{+}, d \tau\right)$ with

$$
\left\{\mathbb{G}_{r}\left(t, \mathbf{x}_{r}\right)\right\}_{a b p q}=\left\{\mathbb{G}_{r}\left(t, \mathbf{x}_{r}\right)\right\}_{p q a b}=\left\{\mathbb{G}_{r}\left(t, \mathbf{x}_{r}\right)\right\}_{a b q p}=\left\{\mathbb{G}_{r}\left(t, \mathbf{x}_{r}\right)\right\}_{b a p q}
$$

Tensor $\mathbb{G}_{0}\left(0, \mathbf{x}_{0}\right)$ is positive-definite but concerning tensor $\mathbb{G}_{r}\left(0, \mathbf{x}_{r}\right)$, we have only proved that there is a positive constant $c$ such that

$$
\left\{\mathbb{G}_{r}\left(0, \mathbf{x}_{r}\right)\right\}_{a b p q}\{\mathbb{X}\}_{a b}\{\mathbb{X}\}_{p q} \geq c\left\{\mathbb{X}^{r e f}\left(\mathbf{x}_{0}\right)\right\}_{a b}\left\{\mathbb{K}^{r e f}\left(\mathbf{x}_{0}\right)\right\}_{a b},
$$

in which $\mathbf{x}_{0}=\left\{\mathbf{X}_{0}^{r e f}\right\}^{-1}\left(\mathbf{x}_{r}\right)$, 敢f $=\frac{1}{\sqrt{\operatorname{det} \mathbb{F}_{0}^{r e f}}} \mathbb{F}_{0}^{r e f} \mathcal{X} \mathbb{F}_{0}^{r e f}$ and where $\mathcal{X}$ is any real symmetric second-order tensor. Equation (53) does not mean that tensor $\mathbb{G}_{r}\left(0, \mathbf{x}_{r}\right)$ is positive-definite.

\section{Weak formulation of the nonlinear steady boundary value problem.}

Let $\mathcal{C}\left(\Omega_{0}\right)$ be the set of sufficiently differentiable functions $\mathbf{v}$ from $\Omega_{0}$ into $\mathbb{R}^{3}$. Let $\mathcal{C}_{a d}\left(\Omega_{0}\right)$ be the admissible function space constituted of all functions $\mathbf{v}$ in $\mathcal{C}\left(\Omega_{0}\right)$ such that $\mathbf{v}=0$ on $\Upsilon_{0}$ and let $\mathcal{C}_{a d, 0}\left(\Omega_{0}\right)$ 
be the subset of all the functions in $\mathcal{C}\left(\Omega_{0}\right)$ such that $\mathbf{v}=0$ on $\Upsilon_{0} \cup \Gamma_{0}$. The weak formulation of the nonlinear steady boundary value problem defined in Section 4 is written as follows : find the displacement field $\mathbf{u}_{0}^{\text {ref }} \in \mathcal{C}_{a d}\left(\Omega_{0}\right)$ with $\mathbf{u}_{0}^{\text {ref }}=\mathbf{u}_{0}^{\text {stat }}$ on $\Gamma_{0}$ such that, for all $\mathbf{v}$ in $\mathcal{C}_{a d, 0}\left(\Omega_{0}\right)$,

$$
k_{e, 0}\left(\mathbf{u}_{0}^{r e f}, \mathbf{v}\right)+k_{c, 0}\left(\mathbf{u}_{0}^{r e f}, \mathbf{v}\right)+k_{2,0}\left(\mathbf{u}_{0}^{r e f}, \mathbf{v}\right)+k_{3,0}\left(\mathbf{u}_{0}^{r e f}, \mathbf{v}\right)-l_{a, 0}\left(\mathbf{u}_{0}^{r e f}, \mathbf{v}\right)=l_{0}(\mathbf{v})
$$

where the symmetric positive-definite bilinear form $k_{e, 0}$, skew symmetric negative bilinear form $k_{c, 0}$, nonlinear forms $k_{2,0}, k_{3,0}, l_{a, 0}$, defined on $\mathcal{C}_{a d}\left(\Omega_{0}\right) \times \mathcal{C}_{a d}\left(\Omega_{0}\right)$, and linear form $l_{0}$ defined on $\mathcal{C}_{a d}\left(\Omega_{0}\right)$, are such that

$$
\begin{aligned}
& k_{e, 0}(\mathbf{u}, \mathbf{v})=\int_{\Omega_{0}}\left\{\mathbb{A}_{0}: \frac{\partial \mathbf{u}}{\partial \mathbf{x}_{0}}\right\}: \frac{\partial \mathbf{v}}{\partial \mathbf{x}_{0}} d \mathbf{x}_{0}, \\
& k_{c, 0}(\mathbf{u}, \mathbf{v})=\int_{\Omega_{0}} \rho_{0}\left\{[R]^{2} \mathbf{u}\right\} \cdot \mathbf{v} d \mathbf{x}_{0}, \\
& k_{2,0}(\mathbf{u}, \mathbf{v})=\frac{1}{2} \int_{\Omega_{0}}\left\{\mathbb{A}_{0}:\left({\frac{\partial \mathbf{u}^{\mathrm{T}}}{\partial \mathbf{x}_{0}}}^{\mathrm{T}} \frac{\partial \mathbf{u}}{\partial \mathbf{x}_{0}}\right)\right\}: \frac{\partial \mathbf{v}}{\partial \mathbf{x}_{0}} d \mathbf{x}_{0}+\int_{\Omega_{0}}\left\{\mathbb{A}_{0}: \frac{\partial \mathbf{u}}{\partial \mathbf{x}_{0}}\right\}:\left\{{\frac{\partial \mathbf{u}^{\mathrm{T}}}{\partial \mathbf{x}_{0}}}^{\frac{\partial \mathbf{v}}{\partial \mathbf{x}_{0}}}\right\} d \mathbf{x}_{0}, \\
& k_{3,0}(\mathbf{u}, \mathbf{v})=\frac{1}{2} \int_{\Omega_{0}}\left\{\mathbb{A}_{0}:\left({\frac{\partial \mathbf{u}^{\mathrm{T}}}{\partial \mathbf{x}_{0}}}^{\mathrm{T}} \frac{\partial \mathbf{u}}{\partial \mathbf{x}_{0}}\right)\right\}:\left\{{\frac{\partial \mathbf{u}^{\mathrm{T}}}{\partial \mathbf{x}_{0}}}^{\frac{\partial \mathbf{v}}{\partial \mathbf{x}_{0}}}\right\} d \mathbf{x}_{0}, \\
& l_{a, 0}(\mathbf{u}, \mathbf{v})=\frac{1}{T} \int_{0}^{+\infty} d \tau \int_{0}^{T} d s \int_{\Sigma_{0}} \mathbf{G}_{0}\left(\tau, s, \mathbf{x}_{0}, \mathbf{u}\right) \cdot \mathbf{v} d S_{0}\left(\mathbf{x}_{0}\right), \\
& l_{0}(\mathbf{v})=\int_{\Omega_{0}} \rho_{0} \mathbf{f}_{0}^{\text {stat }} \cdot \mathbf{v} d \mathbf{x}_{0}-\int_{\Omega_{0}} \rho_{0}\left\{[R]^{2} \mathbf{x}_{0}\right\} \cdot \mathbf{v} d \mathbf{x}_{0} .
\end{aligned}
$$

\section{Finite element discretization of the nonlinear steady boundary value problem.}

Let $\mathcal{C}_{a d, h}\left(\Omega_{0}\right)$ be the subset of $\mathcal{C}_{a d}\left(\Omega_{0}\right)$ corresponding to a finite element discretization of domain $\Omega_{0}$. Let $\left[N_{0}\left(\mathbf{x}_{0}\right)\right]$ be the $3 \times N_{h}$ real interpolation matrix such that the corresponding approximation of $\mathbf{u}_{0}^{r e f}$ and $\mathbf{v}$ (always denoted as $\mathbf{u}_{0}^{\text {ref }}$ and $\mathbf{v}$ ) are written as $\left[N_{0}\left(\mathbf{x}_{0}\right)\right] \mathbf{U}$ and $\left[N_{0}\left(\mathbf{x}_{0}\right)\right] \mathbf{V}$, in which $\mathbf{U}=\left[\begin{array}{l}\mathbf{U}_{\text {stat }}^{d} \\ \mathbf{U}^{f}\end{array}\right]$ and $\mathbf{V}=\left[\begin{array}{l}0 \\ \mathbf{V}^{f}\end{array}\right]$ belong to $\mathbb{R}^{N_{h}}$, where $\mathbf{U}_{\text {stat }}^{d}$ is the vector of the degrees-of-freedom related to the mesh of $\Gamma_{0}$. The finite element approximation of Eq. (54) is written as

$$
\mathbf{V}^{\mathrm{T}}\left[\left[K_{e, 0}\right]+\left[K_{c, 0}\right]+\left[K_{2,0}(\mathbf{U})\right]+\left[K_{3,0}(\mathbf{U})\right]\right] \mathbf{U}=\mathbf{V}^{\mathrm{T}} \mathbf{L}_{0}+\mathbf{V}^{\mathrm{T}} \mathbf{L}_{a}(\mathbf{U})
$$

where the symmetric positive-definite matrices $\left[K_{e, 0}\right],\left[K_{3,0}(\mathbf{U})\right]$, the symmetric negative-definite matrix $\left[K_{c, 0}\right]$, the nonsymmetric matrix $\left[K_{2,0}(\mathbf{U})\right]$ and the vectors $\mathbf{L}_{a, 0}(\mathbf{U}), \mathbf{L}_{0}$ are defined in Appendix A. Introducing the matrices

$$
\left[\begin{array}{cc}
{\left[A_{0}^{d d}\left(\mathbf{U}_{\text {stat }}^{d}, \mathbf{U}^{f}\right)\right]} & {\left[A_{0}^{d f}\left(\mathbf{U}_{\text {stat }}^{d}, \mathbf{U}^{f}\right)\right]} \\
{\left[A_{0}^{f d}\left(\mathbf{U}_{\text {stat }}^{d}, \mathbf{U}^{f}\right)\right]} & {\left[A_{0}^{f f}\left(\mathbf{U}_{\text {stat }}^{d}, \mathbf{U}^{f}\right)\right]}
\end{array}\right]=\left[K_{e, 0}\right]+\left[K_{c, 0}\right]+\left[K_{2,0}(\mathbf{U})\right]+\left[K_{3,0}(\mathbf{U})\right]
$$




$$
\left[\begin{array}{c}
{\left[F_{0}^{d}\left(\mathbf{U}_{\text {stat }}^{d}, \mathbf{U}^{f}\right)\right]} \\
{\left[F_{0}^{f}\left(\mathbf{U}_{\text {stat }}^{d}, \mathbf{U}^{f}\right)\right]}
\end{array}\right]=\mathbf{L}_{a, 0}(\mathbf{U})+\mathbf{L}_{0}
$$

equation (61) yields

$$
\left[A_{0}^{f d}\left(\mathbf{U}_{\text {stat }}^{d}, \mathbf{U}^{f}\right)\right] \mathbf{U}_{\text {stat }}^{d}+\left[A_{0}^{f f}\left(\mathbf{U}_{\text {stat }}^{d}, \mathbf{U}^{f}\right)\right] \mathbf{U}^{f}=\mathbf{F}_{0}^{f}\left(\mathbf{U}_{\text {stat }}^{d}, \mathbf{U}^{f}\right)
$$

Moreover, a method based on Lagrange multipliers could be used in order to take into account the Dirichlet condition $\mathbf{u}_{0}^{\text {ref }}=\mathbf{u}_{0}^{\text {stat }}$ on $\Gamma_{0}[15]$.

\section{Weak formulation of the nonlinear dynamic boundary value problem in the rotating frame with} respect to the reference configuration.

Let $\mathcal{C}\left(\Omega_{r}\right)$ be the set of sufficiently differentiable functions $\mathbf{v}$ from $\Omega_{r}$ into $\mathbb{R}^{3}$. Let $\mathcal{C}_{a d}\left(\Omega_{r}\right)$ be the admissible function space constituted of all functions $\mathbf{v}$ in $\mathcal{C}\left(\Omega_{r}\right)$ such that $\mathbf{v}=0$ on $\Upsilon_{r}$ and let $\mathcal{C}_{a d, 0}\left(\Omega_{r}\right)$ be the subset of all the functions in $\mathcal{C}\left(\Omega_{r}\right)$ such that $\mathbf{v}=0$ on $\Upsilon_{r} \cup \Gamma_{r}$. The weak formulation of the nonlinear dynamic boundary value problem defined in Section 5 is written as follows : find the displacement field $\mathbf{u}_{r}\left(t, \mathbf{x}_{r}\right) \in \mathcal{C}_{a d}\left(\Omega_{r}\right)$ with $\mathbf{u}_{r}\left(t, \mathbf{x}_{r}\right)=\mathbf{u}_{r}^{\operatorname{dir}}\left(t, \mathbf{x}_{r}\right)$ on $\Gamma_{r}$ such that, for all $\mathbf{v}$ in $\mathcal{C}_{a d, 0}\left(\Omega_{r}\right)$,

$$
\begin{aligned}
m_{r}\left(\ddot{\mathbf{u}}_{r}, \mathbf{v}\right) & +c_{r}\left(\dot{\mathbf{u}}_{r}, \mathbf{v}\right)+k_{g, r}\left(\mathbf{u}_{r}, \mathbf{v}\right)+k_{c, r}\left(\mathbf{u}_{r}, \mathbf{v}\right)+k_{e, r}\left(\mathbf{u}_{r}, \mathbf{v}\right)+\frac{1}{2} k_{2, r}\left(\mathbf{u}_{r}, \mathbf{u}_{r}, \mathbf{v}\right)+k_{2, r}\left(\mathbf{v}, \mathbf{u}_{r}, \mathbf{u}_{r}\right) \\
& +k_{3, r}\left(\mathbf{u}_{r}, \mathbf{u}_{r}, \mathbf{u}_{r}, \mathbf{v}\right)+\int_{0}^{+\infty} g_{r}\left(\tau, \mathbf{u}_{r}(t-\tau), \mathbf{v}\right) d \tau+\int_{0}^{+\infty} g_{2, r}\left(\tau, \mathbf{v}, \mathbf{u}_{r}(t), \mathbf{u}_{r}(t-\tau)\right) d \tau \\
& +\frac{1}{2} \int_{0}^{+\infty} g_{2, r}\left(\tau, \mathbf{u}_{r}(t-\tau), \mathbf{u}_{r}(t-\tau), \mathbf{v}\right) d \tau+\int_{0}^{+\infty} g_{3, r}\left(\tau, \mathbf{u}_{r}(t-\tau), \mathbf{u}_{r}(t-\tau), \mathbf{u}_{r}(t), \mathbf{v}\right) d \tau \\
& -\int_{0}^{+\infty} l_{a, r}\left(\tau, t, \mathbf{u}_{r}(t-\tau), \mathbf{v}\right) d \tau=l_{r}(t, \mathbf{v})
\end{aligned}
$$

where, for all $\mathbf{u}, \mathbf{v}, \mathbf{w}$ and $\mathbf{r}$ in $\mathcal{C}_{a d}\left(\Omega_{r}\right)$, we have

$$
\begin{gathered}
k_{g, r}(\mathbf{u}, \mathbf{v})=\int_{\Omega_{r}} \sigma_{r}:\left\{\frac{\partial \mathbf{u}^{\mathrm{T}}}{\partial \mathbf{x}_{r}} \frac{\partial \mathbf{v}}{\partial \mathbf{x}_{r}}\right\} d \mathbf{x}_{r}, \\
k_{e, r}(\mathbf{u}, \mathbf{v})=\int_{\Omega_{r}}\left\{\mathbb{G}_{r}\left(0, \mathbf{x}_{r}\right): \frac{\partial \mathbf{u}}{\partial \mathbf{x}_{r}}\right\}: \frac{\partial \mathbf{v}}{\partial \mathbf{x}_{r}} d \mathbf{x}_{r}, \\
k_{c, r}(\mathbf{u}, \mathbf{v})=\int_{\Omega_{r}} \rho_{r}\left\{[R]^{2} \mathbf{u}\right\} \cdot \mathbf{v} d \mathbf{x}_{r}, \\
g_{r}(t, \mathbf{u}, \mathbf{v})=\int_{\Omega_{r}}\left\{\dot{\mathbb{G}}_{r}\left(t, \mathbf{x}_{r}\right): \frac{\partial \mathbf{u}}{\partial \mathbf{x}_{r}}\right\}: \frac{\partial \mathbf{v}}{\partial \mathbf{x}_{r}} d \mathbf{x}_{r}, \\
c_{r}(\mathbf{u}, \mathbf{v})=2 \int_{\Omega_{r}} \rho_{r}\{[R] \mathbf{u}\} \cdot \mathbf{v} d \mathbf{x}_{r}, \\
m_{r}(\mathbf{u}, \mathbf{v})=\int_{\Omega_{r}} \rho_{r} \mathbf{u} \cdot \mathbf{v} d \mathbf{x}_{r},
\end{gathered}
$$




$$
\begin{gathered}
g_{2, r}(t, \mathbf{u}, \mathbf{v}, \mathbf{w})=\int_{\Omega_{r}}\left\{\dot{\mathbb{G}}_{r}\left(t, \mathbf{x}_{r}\right):\left(\frac{\partial \mathbf{u}^{\mathrm{T}}}{\partial \mathbf{x}_{r}} \frac{\partial \mathbf{v}}{\partial \mathbf{x}_{r}}\right)\right\}: \frac{\partial \mathbf{w}}{\partial \mathbf{x}_{r}} d \mathbf{x}_{r}, \\
g_{3, r}(t, \mathbf{u}, \mathbf{v}, \mathbf{w}, \mathbf{r})=\frac{1}{2} \int_{\Omega_{r}}\left\{\dot{\mathbb{G}}_{r}\left(t, \mathbf{x}_{r}\right):\left(\frac{\partial \mathbf{u}^{\mathrm{T}}}{\partial \mathbf{x}_{r}} \frac{\partial \mathbf{v}}{\partial \mathbf{x}_{r}}\right)\right\}:\left\{\frac{\partial \mathbf{w}^{\mathrm{T}}}{\partial \mathbf{x}_{r}} \frac{\partial \mathbf{r}}{\partial \mathbf{x}_{r}}\right\} d \mathbf{x}_{r}, \\
k_{2, r}(\mathbf{u}, \mathbf{v}, \mathbf{w})=\int_{\Omega_{r}}\left\{\mathbb{G}_{r}\left(0, \mathbf{x}_{r}\right):\left(\frac{\partial \mathbf{u}^{\mathrm{T}}}{\partial \mathbf{x}_{r}} \frac{\partial \mathbf{v}}{\partial \mathbf{x}_{r}}\right)\right\}: \frac{\partial \mathbf{w}}{\partial \mathbf{x}_{r}} d \mathbf{x}_{r}, \\
k_{3, r}(\mathbf{u}, \mathbf{v}, \mathbf{w}, \mathbf{r})=\frac{1}{2} \int_{\Omega_{r}}\left\{\mathbb{G}_{r}\left(0, \mathbf{x}_{r}\right):\left(\frac{\partial \mathbf{u}^{\mathrm{T}}}{\partial \mathbf{x}_{r}} \frac{\partial \mathbf{v}}{\partial \mathbf{x}_{r}}\right)\right\}:\left\{\frac{\partial \mathbf{w}^{\mathrm{T}}}{\partial \mathbf{x}_{r}} \frac{\partial \mathbf{r}}{\partial \mathbf{x}_{r}}\right\} d \mathbf{x}_{r}, \\
l_{a, r}(\tau, t, \mathbf{u}, \mathbf{v})=\int_{\Sigma_{r}} \mathbf{G}_{r}^{d y n}\left(\tau, t, \mathbf{x}_{r},\left\{\mathbf{u}\left(\mathbf{y}_{r}\right)\right\}_{\mathbf{y}_{r} \in \Sigma_{r}}\right) \cdot \mathbf{v} d S_{r} \\
l_{r}(t, \mathbf{v})=\int_{\Omega_{r}} \rho_{r}\left(\mathbf{x}_{r}\right) \mathbf{f}_{r}^{d y n}\left(t, \mathbf{x}_{r}\right) \cdot \mathbf{v} d \mathbf{x}_{r}
\end{gathered}
$$

where

$$
\begin{gathered}
\mathbf{G}_{r}^{\text {dyn }}\left(\tau, t, \mathbf{x}_{r}, \delta \mathbf{u}_{r}\right)=\mathbf{G}_{r}\left(\tau, t, \mathbf{x}_{r}, \delta \mathbf{u}_{r}\right)-\frac{1}{T} \int_{0}^{T} \mathbf{G}_{r}\left(\tau, t, \mathbf{x}_{r}, 0\right) d t \\
\mathbf{f}_{r}^{\text {dyn }}\left(t, \mathbf{x}_{r}\right)=\mathbf{f}_{r}\left(t, \mathbf{x}_{r}\right)-\mathbf{f}_{r}^{\text {stat }}\left(\mathbf{x}_{r}\right)
\end{gathered}
$$

\section{Treatment of the time dependent Dirichlet boundary condition applied to the structure in the} rotating frame

In order to obtain an homogenous Dirichlet condition on $\Gamma_{r}$, the functions $\mathbf{u}_{r}^{\text {tran }}\left(t, \mathbf{x}_{r}\right)$ and $\mathbf{u}_{r}^{\text {hom }}\left(t, \mathbf{x}_{r}\right)$ belonging to $\mathcal{C}_{a d}\left(\Omega_{r}\right)$ are introduced such that $\mathbf{u}_{r}=\mathbf{u}_{r}^{\text {tran }}+\mathbf{u}_{r}^{\text {hom }}$. The function $\mathbf{u}_{r}^{\text {tran }}\left(t, \mathbf{x}_{r}\right)$ is a translation which is defined such that $\mathbf{u}_{r}^{\operatorname{tran}}\left(t, \mathbf{x}_{r}\right)=\mathbf{u}_{r}^{\operatorname{dir}}\left(t, \mathbf{x}_{r}\right)$ on $\Gamma_{r}$ and such that, for all $\mathbf{v}$ in $\mathcal{C}_{a d, 0}\left(\Omega_{r}\right)$

$$
k_{g, r}\left(\mathbf{u}_{r}^{\text {tran }}, \mathbf{v}\right)+k_{c, r}\left(\mathbf{u}_{r}^{\text {tran }}, \mathbf{v}\right)+k_{e, r}\left(\mathbf{u}_{r}^{\text {tran }}, \mathbf{v}\right)=0
$$

It is assumed that Eq. (80) has a unique solution. Substituting $\mathbf{u}_{r}=\mathbf{u}_{r}^{\text {tran }}+\mathbf{u}_{r}^{\text {hom }}$ into Eq. (65), we deduce that $\mathbf{u}_{r}^{\text {hom }}\left(t, \mathbf{x}_{r}\right)$ belongs to $\mathcal{C}_{a d, 0}\left(\Omega_{r}\right)$ is such that, for all $\mathbf{v}$ in $\mathcal{C}_{a d, 0}\left(\Omega_{r}\right)$,

$$
\begin{aligned}
& m_{r}\left(\ddot{\mathbf{u}}_{r}^{h o m}, \mathbf{v}\right)+c_{r}\left(\dot{\mathbf{u}}_{r}^{h o m}, \mathbf{v}\right)+k_{g, r}\left(\mathbf{u}_{r}^{h o m}, \mathbf{v}\right)+k_{c, r}\left(\mathbf{u}_{r}^{h o m}, \mathbf{v}\right)+k_{e, r}\left(\mathbf{u}_{r}^{h o m}, \mathbf{v}\right)+\frac{1}{2} k_{2, r}\left(\mathbf{u}_{r}^{h o m}, \mathbf{u}_{r}^{h o m}, \mathbf{v}\right) \\
& +k_{2, r}\left(\mathbf{v}, \mathbf{u}_{r}^{h o m}, \mathbf{u}_{r}^{h o m}\right)+k_{3, r}\left(\mathbf{u}_{r}^{h o m}, \mathbf{u}_{r}^{h o m}, \mathbf{u}_{r}^{h o m}, \mathbf{v}\right)+\widehat{k}_{g, r}\left(t, \mathbf{u}_{r}^{h o m}, \mathbf{v}\right)+\widehat{k}_{e, r}\left(t, \mathbf{u}_{r}^{h o m}, \mathbf{v}\right) \\
& +\widehat{k}_{2, r}\left(t, \mathbf{u}_{r}^{h o m}, \mathbf{u}_{r}^{h o m}, \mathbf{v}\right)+2 \widehat{k}_{2, r}\left(t, \mathbf{v}, \mathbf{u}_{r}^{h o m}, \mathbf{u}_{r}^{h o m}\right)+\int_{0}^{+\infty} g_{r}\left(\tau, \mathbf{u}_{r}^{h o m}(t-\tau), \mathbf{v}\right) d \tau \\
& +\int_{0}^{+\infty} g_{2, r}\left(\tau, \mathbf{v}, \mathbf{u}_{r}^{h o m}(t), \mathbf{u}_{r}^{h o m}(t-\tau)\right) d \tau+\frac{1}{2} \int_{0}^{+\infty} g_{2, r}\left(\tau, \mathbf{u}_{r}^{h o m}(t-\tau), \mathbf{u}_{r}^{h o m}(t-\tau), \mathbf{v}\right) d \tau \\
& +\int_{0}^{+\infty} g_{3, r}\left(\tau, \mathbf{u}_{r}^{h o m}(t-\tau), \mathbf{u}_{r}^{h o m}(t-\tau), \mathbf{u}_{r}^{h o m}(t), \mathbf{v}\right) d \tau+\int_{0}^{+\infty} \widehat{g}_{r}\left(\tau, t, \mathbf{u}_{r}^{h o m}(t-\tau), \mathbf{v}\right) d \tau \\
& +\int_{0}^{+\infty} \widehat{g}_{2, r}\left(\tau, t, \mathbf{u}_{r}^{h o m}(t-\tau), \mathbf{u}_{r}^{h o m}(t-\tau), \mathbf{v}\right) d \tau+\int_{0}^{+\infty} \widehat{b}_{2, r}\left(\tau, t, \mathbf{u}_{r}^{h o m}(t-\tau), \mathbf{u}_{r}^{h o m}(t), \mathbf{v}\right) d \tau
\end{aligned}
$$




$$
-\int_{0}^{+\infty} \widehat{l}_{a, r}\left(\tau, t, \mathbf{u}_{r}^{h o m}(t-\tau), \mathbf{v}\right) d \tau=l_{r}(t, \mathbf{v})-\widehat{l}_{r}(t, \mathbf{v})
$$

where multilinear forms $\widehat{k}_{g, r}, \widehat{k}_{e, r}, \widehat{g}_{r}, \widehat{g}_{2, r}, \widehat{k}_{2, r}$, nonlinear form $\widehat{l}_{a, r}$ and linear form $\widehat{l}_{r}$ (defined in Appendix B) correspond to the treatment of the time dependent boundary condition. Equation (81) is the translated weak formulation of the nonlinear dynamical boundary value problem.

\section{Finite element discretization of the nonlinear dynamic boundary value problem}

Let $\mathcal{C}_{a d, h}\left(\Omega_{r}\right)$ be the subset of $\mathcal{C}_{a d}\left(\Omega_{r}\right)$ corresponding to a finite element discretization of domain $\Omega_{r}$. Let $\left[N_{r}\left(\mathbf{x}_{r}\right)\right]$ be the $3 \times N_{h}$ real interpolation matrix such that the corresponding approximation of $\mathbf{u}_{r}^{\text {tran }}\left(t, \mathbf{x}_{r}\right), \mathbf{u}_{r}^{\text {hom }}\left(t, \mathbf{x}_{r}\right)$ and $\mathbf{v}$ (always denoted as $\mathbf{u}_{r}^{\text {tran }}\left(t, \mathbf{x}_{r}\right), \mathbf{u}_{r}^{\text {hom }}\left(t, \mathbf{x}_{r}\right)$ and $\mathbf{v}$ ) are written as $\left[N_{r}\left(\mathbf{x}_{r}\right)\right] \mathbf{U}^{\operatorname{tran}}(t),\left[N_{r}\left(\mathbf{x}_{r}\right)\right] \mathbf{U}^{\text {hom }}(t)$ and $\left[N_{r}\left(\mathbf{x}_{r}\right)\right] \mathbf{V}$, in which $\mathbf{U}^{\operatorname{tran}}(t)=\left[\begin{array}{c}\mathbf{U}_{d}^{\text {dir }}(t) \\ \mathbf{U}_{f}^{\text {tran }}(t)\end{array}\right], \mathbf{U}^{\text {hom }}(t)=$ $\left[\begin{array}{l}0 \\ \mathbf{U}_{f}^{\text {hom }}\end{array}\right]$ and $\mathbf{V}=\left[\begin{array}{l}0 \\ \mathbf{V}^{f}\end{array}\right]$ belong to $\mathbb{R}^{N_{h}}$, where $\mathbf{U}_{d}^{d i r}(t)$ is the vector of the degrees-of-freedom related to the mesh of $\Gamma_{r}$. The finite element approximation of Eqs. (80) and (81) is written as

$$
\begin{gathered}
\mathbf{V}^{\mathrm{T}}\left\{\left[K_{g, r}\right]+\left[K_{c, r}\right]+\left[K_{e, r}\right]\right\} \mathbf{U}^{\text {tran }}(t)=0, \\
\mathbf{V}^{\mathrm{T}}\left[M_{r}\right] \ddot{\mathbf{U}}_{t}^{h o m}+\mathbf{V}^{\mathrm{T}}\left[C_{r}\right] \dot{\mathbf{U}}_{t}^{h o m}+\mathbf{V}^{\mathrm{T}}\left\{\left[K_{g, r}\right]+\left[K_{c, r}\right]+\left[K_{e, r}\right]+\left[K_{2, r}\left(\mathbf{U}_{t}^{h o m}\right)\right]^{\mathrm{T}}+\frac{1}{2}\left[K_{2, r}\left(\mathbf{U}_{t}^{h o m}\right)\right]\right. \\
\left.+\left[K_{3, r}\left(\mathbf{U}_{t}^{h o m}, \mathbf{U}_{t}^{h o m}\right)\right]+\left[\widehat{K}_{e, r}(t)\right]+\left[\widehat{K}_{g, r}(t)\right]+\left[\widehat{K}_{2, r}\left(t, \mathbf{U}_{t}^{h o m}\right)\right]+2\left[\widehat{K}_{2, r}\left(t, \mathbf{U}_{t}^{h o m}\right)\right]^{\mathrm{T}}\right\} \mathbf{U}_{t}^{h o m} \\
+\int_{0}^{+\infty} \mathbf{V}^{\mathrm{T}}\left\{\left[G_{r}(\tau)\right]+\left[\widehat{G}_{r}(\tau, t)\right]+\frac{1}{2}\left[G_{2, r}\left(\tau, t, \mathbf{U}_{t-\tau}^{h o m}\right)\right]+\left[G_{2, r}\left(\tau, t, \mathbf{U}_{t}^{h o m}\right)\right]^{\mathrm{T}}+\left[\widehat{B}_{2, r}\left(\tau, t, \mathbf{U}_{t}^{h o m}\right)\right]\right. \\
+\left[\widehat{G}_{2, r}\left(\tau, t, \mathbf{U}_{t-\tau}^{h o m}\right)\right]+\left[\widehat{G}_{3, r}\left(\tau, t, \mathbf{U}_{t}^{h o m}, \mathbf{U}_{t-\tau}^{h o m}\right)\right\} \mathbf{U}_{t-\tau}^{h o m} d \tau-\int_{0}^{+\infty} \mathbf{V}^{\mathrm{T}} \widehat{\mathbf{L}}_{a, h}\left(\tau, t, \mathbf{U}_{t-\tau}^{h o m}\right) d \tau \\
\\
=\mathbf{V}^{\mathrm{T}} \mathbf{L}_{r, h}(t)-\mathbf{V}^{\mathrm{T}} \widehat{\mathbf{L}}_{r, h}(t),
\end{gathered}
$$

with $\mathbf{U}_{t}^{\text {hom }}=\mathbf{U}^{\text {hom }}(t), \mathbf{U}_{t}^{\text {tran }}=\mathbf{U}^{\text {tran }}(t)$ and where, for all $\mathbf{U}, \mathbf{V}$ and $\mathbf{W}$ in $\mathbb{R}^{N_{h}}$, the matrices of Eq. (83) are defined in Appendix C. In a first step, Eq. (82) is solved and allows vector $\mathbf{U}^{\operatorname{tran}}(t)$ to be constructed. In a second step, $\mathbf{U}^{\text {tran }}(t)$ being known, Eq. (83) is solved and gives $\mathbf{U}^{\text {hom }}(t)$. The nonlinear dynamical responses of the rotating structure is then given by $\left[N_{r}\left(\mathbf{x}_{r}\right)\right]\left\{\mathbf{U}^{\text {hom }}(t)+\mathbf{U}^{\text {tran }}(t)\right\}$.

\section{Sensitivity analysis of the nonlinear dynamical problem for small data perturbation}

This section deals with small perturbations of the geometry, mass density and constitutive equation parameters. The nonlinear dynamical equations are written with respect to the reference configuration defined by the domain $\Omega_{r}$. However, perturbations of the geometry, mass density and constitutive equations cannot be defined on $\Omega_{r}$ because this domain is obtained by solving the nonlinear steady boundary value problem defined on Section 4 , and written with respect to domain $\Omega_{0}$. Consequently, 
perturbations have to be defined on the domain $\Omega_{0}$ and rewritten with respect to the domain $\Omega_{r}$. In the sequel, small perturbations of the data is assumed.

For perturbations applied to the geometry, mass density and constitutive equation parameters, the deformed configuration occupies the domain $\Omega^{\prime}(t)$ with boundary $\partial \Omega^{\prime}(t)$ in $\mathcal{R}_{1}$ and at time $t$.

Let $\Omega_{\overline{0}}$ be the domain occupied by the perturbed rotating natural configuration and let $\mathbf{X}_{0 \overline{0}}$ be the mapping from $\Omega_{0}$ on $\Omega_{\overline{0}}$ such that $\operatorname{det} \partial_{\mathbf{x}_{0}} \mathbf{X}_{0 \overline{0}}$ be a positive real-valued function. The geometry perturbation of the rotating natural configuration is represented by the $\mathbb{R}^{3}$-valued displacement field $\mathbf{u}_{0 \overline{0}}$ defined on $\Omega_{0}$ such that

$$
\mathbf{u}_{0 \overline{0}}\left(\mathbf{x}_{0}\right)=\mathbf{X}_{0 \overline{0}}\left(\mathbf{x}_{0}\right)-\mathbf{x}_{0}
$$

Let $\mathbb{F}_{0 \overline{0}}=\partial_{\mathbf{x}_{0}} \mathbf{X}_{0 \overline{0}}$ be defined on domain $\Omega_{0}$. Let $\mathbf{X}_{\overline{0}}^{\prime}\left(t, \mathbf{x}_{\overline{0}}\right)$ be the position in rotating frame $\mathcal{R}_{1}$ and at time $t$ of the particule located at position $\mathbf{x}_{\overline{0}} \in \Omega_{\overline{0}}$ in the perturbed rotating natural configuration observed in $\mathcal{R}_{1}$. Let $\mathbf{X}_{\overline{0}}^{\text {ref }}=\mathbf{X}_{0}^{\text {ref }} \circ\left\{\mathbf{X}_{0 \overline{0}}\right\}^{-1}$ defined from $\Omega_{\overline{0}}$ into $\Omega_{r}$ and let $\mathbf{X}_{r}^{\prime}=\mathbf{X}_{\overline{0}}^{\prime} \circ\left\{\mathbf{X}_{\overline{0}}^{r e f}\right\}^{-1}$ be the mapping from $\Omega_{r}$ into $\Omega^{\prime}(t)$. Let us consider the Green-Lagrange strain tensors $\mathbb{E}_{0}^{\prime}=\frac{1}{2}\left(\mathbb{F}_{\overline{0}}^{\prime}{ }^{\mathrm{T}} \mathbb{F}_{0}^{\prime}-[I]\right)$ and $\mathbb{E}_{\overline{0}}^{r e f}=\frac{1}{2}\left(\mathbb{F}_{\overline{0}}^{r e f^{\mathrm{T}}} \mathbb{F}_{\overline{0}}^{r e f}-[I]\right)$, both defined on $\Omega_{\overline{0}}$ and $\mathbb{E}_{r}^{\prime}=\frac{1}{2}\left(\mathbb{F}_{r}^{\prime \mathrm{T}} \mathbb{F}_{r}^{\prime}-[I]\right)$ defined on $\Omega_{r}$ in which $\mathbb{F}_{\overline{0}}^{\prime}=\partial_{\mathbf{x}_{0}} \mathbf{X}_{\overline{0}}^{\prime}, \mathbb{F}_{\overline{0}}^{r e f}=\partial_{\mathbf{x}_{\overline{0}}} \mathbf{X}_{\overline{0}}^{r e f}$ and $\mathbb{F}_{r}^{\prime}=\partial_{\mathbf{x}_{r}} \mathbf{X}_{r}^{\prime}$.

The mass density perturbation of the rotating natural configuration is given by the real-valued mapping $\mathbf{x}_{\overline{0}} \mapsto \Delta \rho_{\overline{0}}\left(\mathbf{x}_{\overline{0}}\right)$ defined on $\Omega_{\overline{0}}$ such that $\rho_{\overline{0}}^{\prime}=\rho_{0} \circ\left\{\mathbf{X}_{0 \overline{0}}\right\}^{-1}+\Delta \rho_{\overline{0}}$ be a positive real-valued function. Let $\rho_{r}^{\prime}$ be the perturbed mass density defined on domain $\Omega_{r}$ and corresponding to the Lagrangian transport by $\mathbf{X}_{\overline{0}}^{r e f}$ of the mapping $\rho_{\overline{0}}^{\prime}$ defined on $\Omega_{\overline{0}}$. We then have

$$
\rho_{r}^{\prime}\left(\mathbf{x}_{r}\right) \circ \mathbf{X}_{\overline{0}}^{r e f}=\frac{1}{\operatorname{det} \mathbb{F}_{\overline{0}}^{r e f}\left(\mathbf{x}_{\overline{0}}\right)}\left(\rho_{0}\left(\mathbf{x}_{0}\right) \circ\left\{\mathbf{X}_{0 \overline{0}}\right\}^{-1}+\Delta \rho_{\overline{0}}\left(\mathbf{x}_{\overline{0}}\right)\right)
$$

where $\mathbf{x}_{0}=\left\{\mathbf{X}_{0}^{r e f}\right\}^{-1}\left(\mathbf{x}_{r}\right), \mathbf{x}_{\overline{0}}=\left\{\mathbf{X}_{\overline{0}}^{\text {ref }}\right\}^{-1}\left(\mathbf{x}_{r}\right)$.

The constitutive equation perturbation is represented by $\Delta \mathbb{G}_{\overline{0}}\left(\tau, \mathbf{x}_{\overline{0}}\right)$ defined on $\Omega_{\overline{0}}$ such that, for all $i, j, k$ and $h$ fixed in $\{1,2,3\}$, functions $\tau \mapsto\left\{\dot{\mathbb{G}}_{\overline{0}}\left(\tau, \mathbf{x}_{\overline{0}}\right)\right\}_{i j k h}$ belong to $L^{2}\left(\mathbb{R}^{+}, \gamma^{-2} d \tau\right) \cap L^{1}\left(\mathbb{R}^{+}, d \tau\right)$ and such that

$$
\begin{gathered}
\Delta \mathbb{A}_{\overline{0}}\left(\mathbf{x}_{\overline{0}}\right)=\lim _{\tau \rightarrow+\infty}\left|\left\{\Delta \mathbb{G}_{\overline{0}}\left(\tau, \mathbf{x}_{\overline{0}}\right)\right\}_{i j k h}\right|<+\infty, \\
\left\{\Delta \mathbb{G}_{\overline{0}}\right\}_{i j k h}=\left\{\Delta \mathbb{G}_{\overline{0}}\right\}_{k h i j}=\left\{\Delta \mathbb{G}_{\overline{0}}\right\}_{j i k h}=\left\{\Delta \mathbb{G}_{\overline{0}}\right\}_{i j h k}, \\
\sum_{i, j, k, h}^{3}\left(\left\{\mathbb{G}_{0}\left(0, \mathbf{x}_{0}\right)\right\}_{i j k h}+\left\{\Delta \mathbb{G}_{\overline{0}}\left(0, \mathbf{x}_{\overline{0}}\right)\right\}_{i j k h}\right)\{\mathbb{X}\}_{i j}\{\mathbb{X}\}_{k h} \geq c^{\prime}\{\mathbb{X}\}_{i j}\{\mathbb{X}\}_{i j},
\end{gathered}
$$


in which $\mathbf{x}_{0}=\left\{\mathbf{X}_{0 \overline{0}}\right\}^{-1}\left(\mathbf{x}_{\overline{0}}\right)$, where $c^{\prime}$ is a positive real number and where $\mathbb{X}$ is any real symmetric second-order tensor.

Let $\sigma^{\prime}\left(t, \mathbf{x}_{t}^{\prime}\right)$ be the perturbed Cauchy stress tensors defined on $\Omega^{\prime}(t)$ for the deformed configuration with perturbation of the geometry, the mass density and the constitutive equation. Let $\mathbb{U} \frac{\prime}{0}\left(t, \mathbf{x}_{\overline{0}}\right)$ be defined on $\Omega_{\overline{0}}$ as the Piola-Kirchhoff transport by $\mathbf{X}_{\overline{0}}^{\prime}$ of $\sigma^{\prime}\left(t, \mathbf{x}_{t}^{\prime}\right)$. Substituting $\mathbb{G}_{0}\left(t, \mathbf{x}_{0}\right)$ by $\mathbb{G}_{0}\left(\tau, \mathbf{x}_{0}\right)+\Delta \mathbb{G}_{\overline{0}}\left(\tau, \mathbf{x}_{\overline{0}}\right)$, $\mathbb{U T}_{0}\left(t, \mathbf{x}_{0}\right)$ by $\mathbb{W} \frac{\prime}{0}\left(t, \mathbf{x}_{\overline{0}}\right)$ and $\mathbb{E}_{0}\left(t, \mathbf{x}_{0}\right)$ by $\mathbb{E}_{\overline{0}}\left(t, \mathbf{x}_{\overline{0}}\right)$ into Eq. (43) yields

$$
\begin{aligned}
\mathbb{U} T_{\overline{0}}^{\prime}\left(t, \mathbf{x}_{\overline{0}}\right)=\left[\mathbb{G}_{0}\left(0, \mathbf{x}_{0}\right)+\Delta \mathbb{G}_{\overline{0}}^{\prime}\left(0, \mathbf{x}_{\overline{0}}\right)\right]: \mathbb{E}_{\overline{0}}^{\prime}\left(t, \mathbf{x}_{\overline{0}}\right) & +\int_{0}^{+\infty} \Delta \dot{\mathbb{G}}_{\overline{0}}\left(\tau, \mathbf{x}_{\overline{0}}\right): \mathbb{E}_{\overline{0}}^{\prime}\left(t-\tau, \mathbf{x}_{\overline{0}}\right) d \tau \\
& +\int_{0}^{+\infty} \dot{\mathbb{G}}_{0}\left(\tau, \mathbf{x}_{0}\right): \mathbb{E}_{\overline{0}}^{\prime}\left(t-\tau, \mathbf{x}_{\overline{0}}\right) d \tau
\end{aligned}
$$

with $\mathbf{x}_{\overline{0}}=\mathbf{X}_{0 \overline{0}}\left(\mathbf{x}_{0}\right)$. Let $\mathbb{U}_{\overline{0}}^{r e f}$ be the mapping defined on domain $\Omega_{\overline{0}}$ suh that

$$
\mathbb{W}_{\overline{0}}^{r e f}\left(\mathbf{x}_{\overline{0}}\right)=\left[\mathbb{G}_{0}\left(0, \mathbf{x}_{0}\right)+\Delta \mathbb{G}_{\overline{0}}\left(0, \mathbf{x}_{\overline{0}}\right)\right]: \mathbb{E}_{\overline{0}}^{r e f} .
$$

with $\mathbf{x}_{\overline{0}}=\mathbf{X}_{0 \overline{0}}\left(\mathbf{x}_{0}\right)$. Let $\sigma_{r}^{\prime}$ and $\mathbb{T I}_{r}^{\prime}$ be defined on domain $\Omega_{r}$ as the Piola-Kirchhoff transports by $\mathbf{X}_{\overline{0}}^{r e f}$ of mappings $\mathbb{T}_{\overline{0}}^{r e f}$ and $\mathbb{U}_{\overline{0}}^{\prime}$ defined on domain $\Omega_{\overline{0}}$. Substituting $\mathbb{E}_{0}^{r e f}$ by $\mathbb{E}_{\overline{0}}^{r e f}, \mathbb{F}_{0}^{r e f}$ by $\mathbb{E}_{\overline{0}}^{r e f}, \mathbb{A}_{0}\left(\mathbf{x}_{0}\right)$ by $\mathbb{A}_{0}\left(\mathbf{x}_{0}\right)+\Delta \mathbb{A}_{\overline{0}}\left(\mathbf{x}_{\overline{0}}\right)$ and $\mathbf{X}_{0}^{\text {ref }}$ by $\mathbf{X}_{\overline{0}}^{\text {ref }}$ into Eq. (49) yields

$$
\widetilde{\sigma}_{r}^{\prime}\left(\mathbf{x}_{r}\right)=\frac{1}{\operatorname{det} \mathbb{F}_{\overline{0}}^{r e f}\left(\mathbf{x}_{\overline{0}}\right)} \mathbb{F}_{\overline{0}}^{r e f}\left(\mathbf{x}_{\overline{0}}\right)\left\{\left[\mathbb{A}_{0}\left(\mathbf{x}_{0}\right)+\mathbb{A}_{\overline{0}}\left(\mathbf{x}_{\overline{0}}\right)\right]: \mathbb{E}_{\overline{0}}^{r e f}\left(\mathbf{x}_{\overline{0}}\right)\right\} \mathbb{F}_{\overline{0}}^{r e f}\left(\mathbf{x}_{\overline{0}}\right)^{\mathrm{T}},
$$

in which $\mathbf{x}_{0}=\left\{\mathbf{X}_{0}^{r e f}\right\}^{-1}\left(\mathbf{x}_{r}\right), \mathbf{x}_{\overline{0}}=\left\{\mathbf{X}_{0}^{r e f}\right\}^{-1}\left(\mathbf{x}_{r}\right)$. Substituting $\mathbb{W}_{r}$ by $\mathbb{W}_{r}^{\prime}, \sigma_{r}$ by $\sigma_{r}^{\prime}, \mathbb{F}_{0}^{r e f}$ by $\mathbb{F}_{\overline{0}}^{r e f}$, $\mathbb{G}_{0}\left(\tau, \mathbf{x}_{0}\right)$ by $\mathbb{G}_{0}\left(\tau, \mathbf{x}_{0}\right)+\Delta \mathbb{G}_{\overline{0}}\left(\tau, \mathbf{x}_{\overline{0}}\right)$ and $\mathbb{E}_{r}$ by $\mathbb{E}_{r}^{\prime}$ into Eqs. (50) and (51) yields

$$
\begin{aligned}
& \mathbb{U}_{r}^{\prime}\left(t, \mathbf{x}_{r}\right)=\mathbb{G}_{r}^{\prime}\left(\mathbf{x}_{r}\right)+\mathbb{G}_{r}^{\prime}\left(0, \mathbf{x}_{r}\right): \mathbb{E}_{r}^{\prime}\left(t, \mathbf{x}_{r}\right)+\int_{0}^{+\infty} \dot{\mathbb{G}}_{r}^{\prime}\left(\tau, \mathbf{x}_{r}\right): \mathbb{E}_{r}^{\prime}\left(t-\tau, \mathbf{x}_{r}\right) d \tau, \\
&\left\{\mathbb{G}_{r}^{\prime}\left(\tau, \mathbf{x}_{r}\right)\right\}_{a b p q}=\frac{1}{\operatorname{det} \mathbb{F}_{\overline{0}}^{r e f}\left(\mathbf{x}_{\overline{0}}\right)}\left\{\mathbb{F}_{\overline{0}}^{r e f}\left(\mathbf{x}_{\overline{0}}\right)\right\}_{a k}\left\{\mathbb{F}_{\overline{0}}^{r e f}\left(\mathbf{x}_{\overline{0}}\right)\right\}_{b l}\left\{\mathbb{F}_{\overline{0}}^{r e f}\left(\mathbf{x}_{\overline{0}}\right)\right\}_{p m}\left\{\mathbb{F}_{\overline{0}}^{r e f}\left(\mathbf{x}_{\overline{0}}\right)\right\}_{q n} \\
&\left.\times\left(\left\{\mathbb{G}_{0}\left(\tau, \mathbf{x}_{0}\right)\right\}_{k l m n}+\Delta \mathbb{G}_{\overline{0}}\left(\tau, \mathbf{x}_{\overline{0}}\right)\right\}_{k l m n}\right),
\end{aligned}
$$

with $\mathbf{x}_{0}=\left\{\mathbf{X}_{0}^{\text {ref }}\right\}^{-1}\left(\mathbf{x}_{r}\right)$ and $\mathbf{x}_{\overline{0}}=\left\{\mathbf{X}_{\overline{0}}^{\text {ref }}\right\}^{-1}\left(\mathbf{x}_{r}\right)$. The perturbation of the mass density and the constitutive equation induced by perturbations $\mathbf{u}_{0 \overline{0}}, \Delta \rho_{\overline{0}}$ and $\Delta \mathbb{G}_{\overline{0}}$ are represented by functions $\Delta \rho_{r}$, $\Delta \mathbb{G}_{r}$ and $\Delta \sigma_{r}$ defined on domain $\Omega_{r}$. For small perturbations, Eqs (85), (91) and (93) can be linearized with respect to mappings $\mathbf{u}_{0 \overline{0}}, \Delta \rho_{\overline{0}}$ and $\Delta \mathbb{G}_{\overline{0}}$. Since $\partial_{\mathbf{x}_{\overline{0}}}\left\{\mathbf{X}_{0 \overline{0}}\right\}^{-1} \simeq[I]-\partial_{\mathbf{x}_{\overline{0}}} \mathbf{u}_{0 \overline{0}}$, we then have $\mathbb{F}_{\overline{0}}^{r e f} \simeq \mathbb{F}_{0}^{r e f}-\mathbb{F}_{0}^{r e f}\left\{\partial_{\mathbf{x}_{\overline{0}}} \mathbf{u}_{0 \overline{0}}\right\}$ and $\operatorname{det} \mathbb{F}_{\overline{0}}^{r e f} \simeq \operatorname{det} \mathbb{F}_{0}^{r e f}\left(1-d i v_{\mathbf{x}_{0}} \mathbf{u}_{0 \overline{0}}\right)$. Consequently, from Eqs. (85), (91) and (93), we deduce that

$$
\Delta \rho_{r}\left(\mathbf{x}_{r}\right)=\rho_{r}\left(\mathbf{x}_{r}\right) \operatorname{div}_{\mathbf{x}_{0}} \mathbf{u}_{0 \overline{0}}\left(\mathbf{x}_{0}\right)+\frac{1}{\operatorname{det} \mathbb{F}_{0}^{r e f}\left(\mathbf{x}_{0}\right)} \Delta \rho_{\overline{0}}\left(\mathbf{x}_{\overline{0}}\right)
$$




$$
\begin{aligned}
& \Delta \sigma_{r}\left(\mathbf{x}_{r}\right)=\mathbb{K}_{1}\left(\mathbf{x}_{r}\right):: \Delta \mathbb{A}_{\overline{0}}\left(\mathbf{x}_{\overline{0}}\right)+\mathbb{T}_{1}\left(\mathbf{x}_{r}\right): \frac{\partial \mathbf{u}_{0 \overline{0}}\left(\mathbf{x}_{0}\right)}{\partial \mathbf{x}_{0}}+\left(\operatorname{div}_{\mathbf{x}_{0}} \mathbf{u}_{0 \overline{0}}\left(\mathbf{x}_{0}\right)\right) \sigma_{r}\left(\mathbf{x}_{r}\right), \\
& \Delta \mathbb{G}_{r}^{\prime}\left(\mathbf{x}_{r}\right)=\mathbb{K}_{2}\left(\mathbf{x}_{r}\right):: \Delta \mathbb{G}_{\overline{0}}\left(\mathbf{x}_{\overline{0}}\right)+\mathbb{T}_{2}\left(\mathbf{x}_{r}\right): \frac{\partial \mathbf{u}_{0 \overline{0}}\left(\mathbf{x}_{0}\right)}{\partial \mathbf{x}_{0}}+\left(\operatorname{div}_{\mathbf{x}_{0}} \mathbf{u}_{0 \overline{0}}\left(\mathbf{x}_{0}\right)\right) \mathbb{G}_{r}\left(\mathbf{x}_{r}\right),
\end{aligned}
$$

where $\mathbb{K}_{1}, \mathbb{K}_{2}, \mathbb{T}_{1}, \mathbb{T}_{2}$ are tensor-valued functions defined in Apendix D and where $\mathbf{x}_{0}=\left\{\mathbf{X}_{0}^{r e f}\right\}^{-1}\left(\mathbf{x}_{r}\right)$, $\mathbf{x}_{\overline{0}}=\left\{\mathbf{X}_{\overline{0}}^{r e f}\right\}^{-1}\left(\mathbf{x}_{r}\right)$.

\section{Weak formulation of the perturbed nonlinear boundary value problem}

The weak formulation of the nonlinear dynamical boundary value problem with small data perturbations is constructed by substituting $\mathbf{u}_{r}$ by $\mathbf{u}_{r}^{\prime}, \rho_{r}$ by $\rho_{r}^{\prime}$, $\mathbb{T}_{r}$ by $\mathbb{T}_{r}^{\prime}, \mathbb{F}_{r}$ by $\mathbb{F}_{r}^{\prime}$ into Eq. (31) and Eq. (92). The weak formulation of the perturbation of the nonlinear dynamic boundary value problem defined in Section 5, is written as follows : find displacement field $\mathbf{u}_{r}^{\prime}\left(t, \mathbf{x}_{r}\right) \in \mathcal{C}_{a d}\left(\Omega_{r}\right)$ with $\mathbf{u}_{r}^{\prime}\left(t, \mathbf{x}_{r}\right)=\mathbf{u}_{r}^{\operatorname{dir}}\left(t, \mathbf{x}_{r}\right)$ on $\Gamma_{r}$ such that, for all $\mathbf{v}$ in $\mathcal{C}_{a d, 0}\left(\Omega_{r}\right)$,

$$
\begin{aligned}
\int_{\Omega_{r}} \rho_{r}^{\prime}\left\{\ddot{\mathbf{u}}_{r}^{\prime}\right\} \cdot \mathbf{v} d \mathbf{x}_{r} & +2 \int_{\Omega_{r}}\left\{\rho_{r}^{\prime}[R] \dot{\mathbf{u}}_{r}^{\prime}\right\} \cdot \mathbf{v} d \mathbf{x}_{r} \\
& +\int_{\Omega_{r}} \sigma_{r}^{\prime}:\left\{\mathbb{F}_{r}^{\prime \mathrm{T}} \frac{\partial \mathbf{v}}{\partial \mathbf{x}_{r}}\right\} d \mathbf{x}_{r}+\int_{\Omega_{r}}\left\{\mathbb{G}_{r}^{\prime}\left(0, \mathbf{x}_{r}\right): \mathbb{E}_{r}^{\prime}\right\}:\left\{\mathbb{F}_{r}^{\prime \mathrm{T}} \frac{\partial \mathbf{v}}{\partial \mathbf{x}_{r}}\right\} d \mathbf{x}_{r} \\
& +\int_{\Omega_{r}} \int_{0}^{+\infty}\left\{\dot{\mathbb{G}}_{r}^{\prime}\left(\tau, \mathbf{x}_{r}\right): \mathbb{E}_{r}^{\prime}\left(t-\tau, \mathbf{x}_{r}\right)\right\}:\left\{\mathbb{F}_{r}^{\prime \mathrm{T}} \frac{\partial \mathbf{v}}{\partial \mathbf{x}_{r}}\right\} d \mathbf{x}_{r} d \tau-\int_{\Omega_{r}} \sigma_{r}^{\prime}: \frac{\partial \mathbf{v}}{\partial \mathbf{x}_{r}} d \mathbf{x}_{r} \\
& +\int_{\Omega_{r}} \rho_{r}^{\prime}\left\{[R]^{2} \mathbf{u}_{r}^{\prime}\right\} \cdot \mathbf{v} d \mathbf{x}_{r}=\int_{\Omega_{r}} \rho_{r}^{\prime} \mathbf{f}_{r}^{d y n \prime} \cdot \mathbf{v} d \mathbf{x}_{r}+\int_{\Sigma_{r}} \mathbf{F}_{r}^{d y n \prime} \cdot \mathbf{v} d S_{r}
\end{aligned}
$$

where $\mathbf{f}_{r}^{\text {dyn' }}\left(t, \mathbf{x}_{r}\right)$ defined on $\Omega_{r}$ and $\mathbf{F}_{r}^{\text {dyn' }}\left(t, \mathbf{x}_{r}\right)$ defined on $\Sigma_{r}$ are such that

$$
\begin{gathered}
\mathbf{f}_{r}^{d y n^{\prime}}\left(t, \mathbf{x}_{r}\right)=\mathbf{f}_{r}-[R]^{2} \mathbf{x}_{r}+\rho_{r}^{\prime}\left(\mathbf{x}_{r}\right)^{-1} \operatorname{div}_{\mathbf{x}_{r}} \sigma_{r}^{\prime}\left(\mathbf{x}_{r}\right) \\
\mathbf{F}_{r}^{d y n^{\prime}}\left(t, \mathbf{x}_{r}\right)=\int_{0}^{+\infty} \mathbf{G}_{r}^{d y n}\left(\tau, t, \mathbf{x}_{r},\left\{\mathbf{u}_{r}^{\prime}\left(t-\tau, \mathbf{y}_{r}\right)\right\}_{\mathbf{y}_{r} \in \Sigma_{r}}\right) d \tau-\Delta \sigma_{r}\left(\mathbf{x}_{r}\right) \mathbf{n}_{r}\left(\mathbf{x}_{r}\right) .
\end{gathered}
$$

14. Treatment of the time dependent Dirichlet boundary condition applied to the perturbed structure in the rotating frame

In order to obtain a homogeneous Dirichlet condition on $\Gamma_{r}$, we substitute $\mathbf{u}_{r}^{\prime}=\mathbf{u}^{\text {hom' }}+\mathbf{u}_{r}^{\text {tran }}$ into Eq. (97) where $\mathbf{u}^{\text {hom }} \in \mathcal{C}_{a d, 0}\left(\Omega_{r}\right)$ is the new unknown of the perturbed nonlinear dynamical problem. We then have

$$
\begin{aligned}
& \left\{\mathbf{M}_{r}+\Delta \mathbf{M}_{r}\right\} \ddot{\mathbf{u}}_{r}^{h o m \prime}(t)+\left\{\mathbf{C}_{r}+\Delta \mathbf{C}_{r}\right\} \dot{\mathbf{u}}_{r}^{h o m \prime}(t)+\left\{\mathbf{K}_{g, r}+\Delta \mathbf{K}_{g, r}+\mathbf{K}_{c, r}+\Delta \mathbf{K}_{c, r}+\mathbf{K}_{e, r}+\Delta \mathbf{K}_{e, r}\right. \\
& +\frac{1}{2} \mathbf{K}_{2, r}\left(\mathbf{u}_{r}^{h o m \prime}(t)\right)+\frac{1}{2} \Delta \mathbf{K}_{2, r}\left(\mathbf{u}_{r}^{h o m \prime}(t)\right)+{ }^{\mathrm{t}} \mathbf{K}_{2, r}\left(\mathbf{u}_{r}^{h o m \prime}(t)\right)+{ }^{\mathrm{t}} \Delta \mathbf{K}_{2, r}\left(\mathbf{u}_{r}^{\text {hom' }}(t)\right) \\
& +\mathbf{K}_{3, r}\left(\mathbf{u}_{r}^{h o m \prime}(t), \mathbf{u}_{r}^{h o m \prime}(t)\right)+\Delta \mathbf{K}_{3, r}\left(\mathbf{u}_{r}^{h o m \prime}(t), \mathbf{u}_{r}^{h o m \prime}(t)\right)+\widehat{\mathbf{K}}_{g, r}(t)+\Delta \widehat{\mathbf{K}}_{g, r}(t)+\widehat{\mathbf{K}}_{e, r}(t)+\Delta \widehat{\mathbf{K}}_{e, r}(t) \\
& \left.+\widehat{\mathbf{K}}_{2, r}\left(t, \mathbf{u}_{r}^{h o m \prime}(t)\right)+\Delta \widehat{\mathbf{K}}_{2, r}\left(t, \mathbf{u}_{r}^{h o m \prime}(t)\right)+2{ }^{t} \widehat{\mathbf{K}}_{2, r}\left(t, \mathbf{u}_{r}^{h o m \prime}(t)\right)+2{ }^{t} \Delta \widehat{\mathbf{K}}_{2, r}\left(t, \mathbf{u}_{r}^{h o m \prime}(t)\right)\right\} \mathbf{u}_{r}^{h o m \prime}(t)
\end{aligned}
$$




$$
\begin{gathered}
+\int_{0}^{+\infty}\left\{\mathbf{G}_{r}(\tau)+\Delta \mathbf{G}_{r}(\tau)+\widehat{\mathbf{G}}_{r}(\tau, t)+\Delta \widehat{\mathbf{G}}_{r}(\tau, t)+\widehat{\mathbf{B}}_{2, r}\left(t, \mathbf{u}_{r}^{h o m \prime}(t)\right)+\Delta \widehat{\mathbf{B}}_{2, r}\left(\tau, t, \mathbf{u}_{r}^{h o m \prime}(t)\right)\right. \\
+\frac{1}{2} \mathbf{G}_{2, r}\left(\tau, \mathbf{u}_{r}^{h o m \prime}(t-\tau)\right)+\frac{1}{2} \Delta \mathbf{G}_{2, r}\left(\tau, \mathbf{u}_{r}^{h o m \prime}(t-\tau)\right)+{ }^{\mathrm{t}} \mathbf{G}_{2, r}\left(\tau, \mathbf{u}_{r}^{h o m \prime}(t)\right)+{ }^{\mathrm{t}} \Delta \mathbf{G}_{2, r}\left(\tau, \mathbf{u}_{r}^{h o m \prime}(t)\right) \\
+\widehat{\mathbf{G}}_{2, r}\left(\tau, t, \mathbf{u}_{r}^{h o m \prime}(t-\tau)\right)+\Delta \widehat{\mathbf{G}}_{2, r}\left(\tau, t, \mathbf{u}_{r}^{h o m \prime}(t-\tau)\right)+\mathbf{G}_{3, r}\left(\tau, \mathbf{u}_{r}^{h o m \prime}(t), \mathbf{u}_{r}^{h o m \prime}(t-\tau)\right) \\
\left.+\Delta \mathbf{G}_{3, r}\left(\tau, \mathbf{u}_{r}^{h o m \prime}(t), \mathbf{u}_{r}^{h o m \prime}(t-\tau)\right)\right\} \mathbf{u}_{r}^{h o m \prime}(t-\tau) d \tau-\int_{0}^{\infty} \mathbf{L}_{a, r}\left(\tau, t, \mathbf{u}_{r}^{h o m \prime}(t-\tau)\right) d \tau \\
=\Delta \mathbf{L}_{a, r}+\mathbf{L}_{r}(t)+\Delta \mathbf{L}_{r}(t)-\widehat{\mathbf{L}}_{r}(t)-\Delta \widehat{\mathbf{L}}_{r}(t),
\end{gathered}
$$

where all multilinear operators in Eq. (99) are defined in Appendix E by their associated multilinear forms.

\section{Formal perturbations methods for solving nonlinear dynamical problem with perturbed data}

Operatorial equation (99) could be rewritten as

$$
\begin{aligned}
\mathbf{M}_{r} \ddot{\mathbf{u}}_{r}^{h o m \prime}(t) & +\mathbf{C}_{r} \dot{\mathbf{u}}_{r}^{h o m \prime}(t)+\mathbf{A}_{r}\left(t, \mathbf{u}_{r}^{h o m \prime}\right)+\widehat{\mathbf{A}}_{r}\left(t, \mathbf{u}_{r}^{h o m \prime}\right) \\
& +\varepsilon\left\{\Delta \mathbf{M}_{r} \ddot{\mathbf{u}}_{r}^{\text {hom' }}(t)+\Delta \mathbf{C}_{r} \dot{\mathbf{u}}_{r}^{\text {hom' }}(t)+\Delta \mathbf{A}_{r}\left(t, \mathbf{u}_{r}^{h o m \prime}\right)+\Delta \widehat{\mathbf{A}}_{r}\left(t, \mathbf{u}_{r}^{h o m \prime}\right)\right\}=0,
\end{aligned}
$$

where operators $\mathbf{A}_{r}, \widehat{\mathbf{A}}_{r}, \Delta \mathbf{A}_{r}$ and $\Delta \widehat{\mathbf{A}}_{r}$ are defined in Appendix $\mathrm{F}$ and where $\varepsilon$ is a nondimensional parameter introduced in order to use a perturbation method [1, 5, 27, 29, 34, 35, 36] for Eq. (100). It should be noted that operators $\widehat{\mathbf{A}}_{r}$ and $\Delta \widehat{\mathbf{A}}_{r}$ correspond to the treatment of the time dependent Dirichlet condition for the perturbed nonlinear dynamical system. The perturbation method considered in this paper is formal and consists in using the asymptotic approximation $\mathbf{u}_{r}^{\text {hom }} \simeq \sum_{n=0}^{+\infty} \varepsilon^{n} \mathbf{u}_{r}^{n}$. It is also well known that secular terms could occur due to solution $\mathbf{u}_{r}^{0}$ of the unperturbed problem. Other more advanced methods (such as the normal form method) can be used to avoid secular terms $[25,26,27,29$, $30,32,33,34]$, but such an application for the present problem is really tricky due to the dimension of the problem which is infinite. It is also assumed that the following expansion can be written,

$$
\widehat{\mathbf{L}}_{a, r}\left(\tau, t, \mathbf{u}_{r}^{0}+\mathbf{u}\right)=\widehat{\mathbf{L}}_{a, r}\left(\tau, t, \mathbf{u}_{r}^{0}\right)+\widehat{\mathbf{L}}_{a, r}^{1}(\tau, t) \mathbf{u}+\widehat{\mathbf{L}}_{a, r}^{2}(\tau, t, \mathbf{u}, \mathbf{u})+\widehat{\mathbf{L}}_{a, r}^{3}(\tau, t, \mathbf{u}, \mathbf{u}, \mathbf{u})+\ldots
$$

Substituting $\mathbf{u}_{r}^{\text {hom' }}=\sum_{n=0}^{+\infty} \varepsilon^{n} \mathbf{u}_{r}^{n}$ into Eq. (100) with $\mathbf{u}_{r}^{0}=\mathbf{u}_{r}^{\text {hom }}$ and identifying terms in $\varepsilon^{n}$, for each integer $n>0$, we then obtain

$$
\begin{aligned}
\mathbf{M}_{r} \ddot{\mathbf{u}}_{r}^{n}(t)+\mathbf{C}_{r} \dot{\mathbf{u}}_{r}^{n}(t) & +\left\{\mathbf{K}_{r}+\mathbf{K}_{r}^{0}(t)+\widehat{\mathbf{K}}_{r}^{0}(t)\right\} \mathbf{u}_{r}^{n}(t)+\int_{0}^{+\infty}\left\{\mathbf{G}_{r}^{0}(\tau, t)+\widehat{\mathbf{G}}_{r}^{0}(\tau, t)\right\} \mathbf{u}_{r}^{n}(t-\tau) d \tau \\
& -\int_{0}^{\infty} \widehat{\mathbf{L}}_{a, r}^{1}(\tau, t) \mathbf{u}_{r}^{n}(t-\tau) d \tau=-\mathbf{F}_{r}^{n}(t)-\widehat{\mathbf{F}}_{r}^{n}(t)
\end{aligned}
$$

where for $n$ fixed into $\{1,2\}, \mathbf{K}_{r}, \mathbf{K}_{r}^{0}(t), \widehat{\mathbf{K}}_{r}^{0}(t), \mathbf{G}_{r}^{0}(\tau, t), \widehat{\mathbf{G}}_{r}^{0}(\tau, t), \mathbf{F}_{r}^{n}(t)$ and $\widehat{\mathbf{F}}_{r}^{n}(t)$ are defined in Appendix F. The finite element approximation of $\mathbf{u}_{r}^{n}$ with $n>0$ is defined as $\left[N_{r}\left(\mathbf{x}_{r}\right)\right] \mathbf{U}^{n}(t)$ with 
$\mathbf{U}^{n}(t)=\left[\begin{array}{l}0 \\ \mathbf{U}_{f}^{n}(t)\end{array}\right]$ and belongs to $\mathcal{C}_{a d, h}\left(\Omega_{r}\right)$. Moreover, the finite element discretization of operators $\widehat{\mathbf{L}}_{a, r}^{1}(\tau, t), \mathbf{K}_{r}, \mathbf{K}_{r}^{0}(t), \widehat{\mathbf{K}}_{r}^{0}(t), \mathbf{G}_{r}^{0}(\tau, t), \widehat{\mathbf{G}}_{r}^{0}(\tau, t), \mathbf{F}_{r}^{n}(t)$ and $\widehat{\mathbf{F}}_{r}^{n}(t)$ with $n$ in $\{1,2\}$ are denoted as $\widehat{\mathbf{L}}_{a, h}^{1}(\tau, t),\left[K_{r}\right],\left[K_{r}^{0}(t)\right],\left[\widehat{K}_{r}^{0}(t)\right],\left[G_{r}^{0}(\tau, t)\right],\left[\widehat{G}_{r}^{0}(\tau, t)\right], \mathbf{F}_{r, h}^{n}(t)$ and $\widehat{\mathbf{F}}_{r, h}^{n}(t)$ and are defined in Appendix G. Consequently, the finite element discretization of Eq. (102) is

$$
\begin{aligned}
{\left[M_{r}\right] \ddot{\mathbf{U}}_{t}^{n}+\left[C_{r}\right] \dot{\mathbf{U}}_{t}^{n} } & +\left\{\left[K_{r}\right]+\left[K_{r}^{0}(t)\right]+\left[\widehat{K}_{r}^{0}(t)\right]\right\} \mathbf{U}_{t}^{n}+\int_{0}^{+\infty}\left\{\left[G_{r}^{0}(\tau, t)\right]+\left[\widehat{G}_{r}^{0}(\tau, t)\right]\right\} \mathbf{U}_{t-\tau}^{n} d \tau \\
& -\int_{0}^{\infty} \widehat{\mathbf{L}}_{a, h}^{1}(\tau, t) \mathbf{U}_{t-\tau}^{n} d \tau=-\mathbf{F}_{r, h}^{n}(t)-\widehat{\mathbf{F}}_{r, h}^{n}(t)
\end{aligned}
$$

with $\mathbf{U}_{t}^{n}=\mathbf{U}^{n}(t)$.

\section{Conclusions}

In general, for nonlinear elastodynamic equations of rotating structures in finite displacement, approximated theories are used. In such a model, it is difficult to introduce viscoelasticity for nonisotropic materials. In this paper, we have presented the details of a complete and exact three-dimensional formulation for nonlinear dynamics in finite displacement of rotating structures constituted of viscoelastic nonisotropic and nonhomogenous materials. In addition, time dependent boundary conditions are considered and the rotating structure is subjected to a complex loading depending on the time history of the structural displacement field (for instance, unsteady aerodynamic forces). A strategy for analyzing such a problem is proposed and nonlinear dynamical matrix equations resulting from the finite element discretization are given. Finally, all the equations concerning sensitivity analysis of the nonlinear dynamical problem system with respect to the perturbed data are given.

Appendix A. Definition of the finite element matrices for the nonlinear steady boundary value problem

$$
\begin{gathered}
{\left[K_{c, 0}\right]=\int_{\Omega_{0}} \rho_{0}\left[N_{0}\left(\mathbf{x}_{0}\right)\right]^{\mathrm{T}}[R]^{2}\left[N_{0}\left(\mathbf{x}_{0}\right)\right] d \mathbf{x}_{0},\left[K_{e, 0}\right]=\int_{\Omega_{0}}\left[B_{0}\left(\mathbf{x}_{0}\right)\right]^{\mathrm{T}}\left[C_{e, 0}\left(\mathbf{x}_{0}\right)\right]\left[B_{0}\left(\mathbf{x}_{0}\right)\right] d \mathbf{x}_{0},} \\
{\left[K_{2,0}(\mathbf{U})\right]=\frac{1}{2} \int_{\Omega_{0}}\left[B_{0}\left(\mathbf{x}_{0}\right)\right]^{\mathrm{T}}\left[C_{e, 0}\left(\mathbf{x}_{0}\right)\right]\left[B_{0}^{N L}\left(\mathbf{x}_{0}, \mathbf{U}\right)\right] d \mathbf{x}_{0}} \\
+\int_{\Omega_{0}}\left[B_{0}^{N L}\left(\mathbf{x}_{0}, \mathbf{U}\right)\right]^{\mathrm{T}}\left[C_{e, 0}\left(\mathbf{x}_{0}\right)\right]\left[B_{0}\left(\mathbf{x}_{0}\right)\right] d \mathbf{x}_{0} \\
{\left[K_{3,0}(\mathbf{U})\right]=\frac{1}{2} \int_{\Omega_{0}}\left[B_{0}^{N L}\left(\mathbf{x}_{0}, \mathbf{U}\right)\right]^{\mathrm{T}}\left[C_{e, 0}\left(\mathbf{x}_{0}\right)\right]\left[B_{0}^{N L}\left(\mathbf{x}_{0}, \mathbf{U}\right)\right] d \mathbf{x}_{0}} \\
\mathbf{L}_{a, 0}(\mathbf{U})=\frac{1}{T} \int_{0}^{+\infty} d \tau \int_{0}^{T} d s \int_{\Sigma_{0}}\left[N_{0}\left(\mathbf{x}_{0}\right)\right]^{\mathrm{T}} \mathbf{G}_{0}\left(\tau, s, \mathbf{x}_{0},\left[N_{0}\left(\mathbf{x}_{0}\right)\right] \mathbf{U}\right) d S_{0}
\end{gathered}
$$




$$
\mathbf{L}_{0}=\int_{\Omega_{0}} \rho_{0}\left(\mathbf{x}_{0}\right)\left[N_{0}\left(\mathbf{x}_{0}\right)\right]^{\mathrm{T}} \mathbf{f}_{0}^{s t a t} d \mathbf{x}_{0}-\int_{\Omega_{0}} \rho\left(\mathbf{x}_{0}\right)\left[N_{0}\left(\mathbf{x}_{0}\right)\right]^{\mathrm{T}}\left\{[R]^{2} \mathbf{x}_{0}\right\} d \mathbf{x}_{0}
$$

where

$$
\begin{gathered}
{[d]=\left[\begin{array}{rrr}
1 & 4 & 6 \\
5 & 2 & 8 \\
7 & 9 & 3
\end{array}\right], \quad\left[B_{0}\left(\mathbf{x}_{0}\right)\right]_{d_{i j} p}=\frac{\partial\left[N_{0}\left(\mathbf{x}_{0}\right)\right]_{i p}}{\partial\left\{\mathbf{x}_{0}\right\}_{j}}} \\
{\left[B_{0}^{N L}\left(\mathbf{x}_{0}, \mathbf{a}\right)\right]_{d_{i j} p}=\frac{\partial\left[N_{0}\left(\mathbf{x}_{0}\right)\right]_{k p}}{\partial\left\{\mathbf{x}_{0}\right\}_{j}} \frac{\partial\left[N_{0}\left(\mathbf{x}_{0}\right)\right]_{k q}}{\partial\left\{\mathbf{x}_{0}\right\}_{i}}\{\mathbf{a}\}_{q}, \quad\left[C_{e, 0}\left(\mathbf{x}_{0}\right)\right]_{d_{i j} d_{k h}}=\left\{\mathbb{A}_{0}\left(\mathbf{x}_{0}\right)\right\}_{i j k h} .}
\end{gathered}
$$

Appendix B. Definition of the multilinear forms for the translated weak formulation of the nonlinear dynamic boundary value problem

$$
\begin{aligned}
& \widehat{k}_{g, r}(t, \mathbf{u}, \mathbf{v})=\int_{\Omega_{r}} \int_{0}^{+\infty}\left\{\dot{\mathbb{G}}_{r}\left(\tau, \mathbf{x}_{r}\right): \mathbb{E}_{r}^{\operatorname{tran}}\left(t-\tau, \mathbf{x}_{r}\right)\right\}:\left\{\frac{\partial \mathbf{u}^{\mathrm{T}}}{\partial \mathbf{x}_{r}} \frac{\partial \mathbf{v}}{\partial \mathbf{x}_{r}}\right\} d \mathbf{x}_{r} d \tau \\
& +\int_{\Omega_{r}}\left\{\mathbb{G}_{r}\left(0, \mathbf{x}_{r}\right): \mathbb{E}_{r}^{\operatorname{tran}}\left(t, \mathbf{x}_{r}\right)\right\}:\left\{\frac{\partial \mathbf{u}^{\mathrm{T}}}{\partial \mathbf{x}_{r}} \frac{\partial \mathbf{v}}{\partial \mathbf{x}_{r}}\right\} d \mathbf{x}_{r} \\
& \widehat{k}_{e, r}(t, \mathbf{u}, \mathbf{v})=k_{2, r}\left(\mathbf{u}, \mathbf{u}_{r}^{\text {tran }}(t), \mathbf{v}\right)+k_{2, r}\left(\mathbf{v}, \mathbf{u}_{r}^{\text {tran }}(t), \mathbf{u}\right)+2 k_{3, r}\left(\mathbf{u}, \mathbf{u}_{r}^{\text {tran }}(t), \mathbf{u}_{r}^{\text {tran }}(t), \mathbf{v}\right),(112) \\
& \widehat{g}_{r}(\tau, t, \mathbf{u}, \mathbf{v})=g_{2, r}\left(\tau, \mathbf{v}, \mathbf{u}_{r}^{\text {tran }}(t), \mathbf{u}\right)+g_{2, r}\left(\tau, \mathbf{u}, \mathbf{u}_{r}^{\operatorname{tran}}(t-\tau), \mathbf{v}\right) \\
& +2 g_{3, r}\left(\tau, \mathbf{u}_{r}^{\operatorname{tran}}(t-\tau), \mathbf{u}, \mathbf{u}_{r}^{\operatorname{tran}}(t), \mathbf{v}\right), \\
& \widehat{b}_{2, r}(\tau, t, \mathbf{u}, \mathbf{v}, \mathbf{w})=2 g_{3, r}\left(\tau, \mathbf{u}_{r}^{\operatorname{tran}}(t-\tau), \mathbf{u}, \mathbf{v}, \mathbf{w}\right) \text {, } \\
& \widehat{k}_{2, r}(t, \mathbf{u}, \mathbf{v}, \mathbf{w})=k_{3, r}\left(\mathbf{u}, \mathbf{v}, \mathbf{u}_{r}^{\operatorname{tran}}(t), \mathbf{w}\right), \\
& \widehat{g}_{2, r}(\tau, t, \mathbf{u}, \mathbf{v}, \mathbf{w})=g_{3, r}\left(\tau, \mathbf{u}, \mathbf{v}, \mathbf{u}_{r}^{\text {tran }}(t), \mathbf{w}\right), \\
& \widehat{l}_{a, r}(\tau, t, \mathbf{u}, \mathbf{v})=l_{a, r}\left(\tau, t, \mathbf{u}_{r}^{\text {tran }}(t-\tau)+\mathbf{u}, \mathbf{v}\right), \\
& \widehat{l}_{r}(t, \mathbf{v})=\frac{1}{2} k_{2, r}\left(\mathbf{u}_{r}^{\text {tran }}, \mathbf{u}_{r}^{\text {tran }}, \mathbf{v}\right)+k_{2, r}\left(\mathbf{v}, \mathbf{u}_{r}^{\text {tran }}, \mathbf{u}_{r}^{\text {tran }}\right)+k_{3, r}\left(\mathbf{u}_{r}^{\text {tran }}, \mathbf{u}_{r}^{\text {tran }}, \mathbf{u}_{r}^{\text {tran }}, \mathbf{v}\right)+c_{r}\left(\dot{\mathbf{u}}_{r}^{\text {tran }}, \mathbf{v}\right) \\
& +m_{r}\left(\ddot{\mathbf{u}}_{r}^{\text {tran }}, \mathbf{v}\right)+\int_{0}^{+\infty} g_{r}\left(\tau, \mathbf{u}_{r}^{\text {tran }}(t-\tau), \mathbf{v}\right) d \tau+\int_{0}^{+\infty} g_{2, r}\left(\tau, \mathbf{v}, \mathbf{u}_{r}^{\text {tran }}(t), \mathbf{u}_{r}^{\text {tran }}(t-\tau)\right) d \tau \\
& +\frac{1}{2} \int_{0}^{+\infty} g_{2, r}\left(\tau, \mathbf{u}_{r}^{\operatorname{tran}}(t-\tau), \mathbf{u}_{r}^{\operatorname{tran}}(t-\tau), \mathbf{v}\right) d \tau \\
& +\int_{0}^{+\infty} g_{3, r}\left(\tau, \mathbf{u}_{r}^{\operatorname{tran}}(t-\tau), \mathbf{u}_{r}^{\operatorname{tran}}(t-\tau), \mathbf{u}_{r}^{\operatorname{tran}}(t), \mathbf{v}\right) d \tau
\end{aligned}
$$

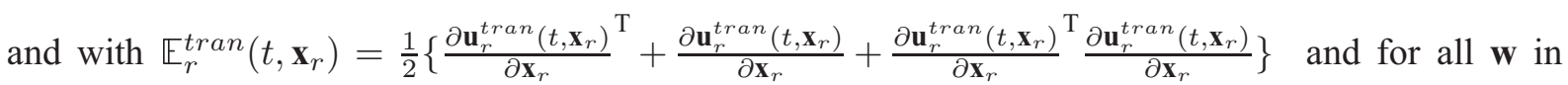
$\mathcal{C}_{a d}\left(\Omega_{r}\right), \widehat{k}_{g, r}(t, \cdot), \widehat{k}_{e, r}(t, \cdot), \widehat{g}_{r}(\tau, t, \cdot),(\mathbf{u}, \mathbf{v}) \mapsto \widehat{b}_{2, r}(\tau, t, \mathbf{w}, \mathbf{u}, \mathbf{v}),(\mathbf{u}, \mathbf{v}) \mapsto \widehat{g}_{2, r}(\tau, t, \mathbf{u}, \mathbf{v}, \mathbf{w})$ and $(\mathbf{u}, \mathbf{v}) \mapsto \widehat{k}_{2, r}(t, \mathbf{u}, \mathbf{v}, \mathbf{w})$ are symmetric bilinear forms. 


\section{Appendix C. Definition of the finite element matrices for the nonlinear dynamical boundary value} problem

$$
\begin{aligned}
& {\left[K_{c, r}\right]=\int_{\Omega_{r}} \rho_{r}\left[N_{r}\left(\mathbf{x}_{r}\right)\right]^{\mathrm{T}}[R]^{2}\left[N_{r}\left(\mathbf{x}_{r}\right)\right] d \mathbf{x}_{r},} \\
& {\left[K_{g, r}\right]=\int_{\Omega_{r}}\left[B_{r}\left(\mathbf{x}_{r}\right)\right]^{\mathrm{T}}\left[C_{g, r}\left(\mathbf{x}_{r}\right)\right]\left[B_{r}\left(\mathbf{x}_{r}\right)\right] d \mathbf{x}_{r}} \\
& {\left[K_{e, r}\right]=\int_{\Omega_{r}}\left[B_{r}\left(\mathbf{x}_{r}\right)\right]^{\mathrm{T}}\left[C_{e, r}\left(0, \mathbf{x}_{r}\right)\right]\left[B_{r}\left(\mathbf{x}_{r}\right)\right] d \mathbf{x}_{r},} \\
& {\left[K_{2, r}(\mathbf{V})\right]=\int_{\Omega_{r}}\left[B_{r}\left(\mathbf{x}_{r}\right)\right]^{\mathrm{T}}\left[C_{e, r}\left(0, \mathbf{x}_{r}\right)\right]\left[B_{r}^{N L}\left(\mathbf{x}_{r}, \mathbf{V}\right)\right] d \mathbf{x}_{r},} \\
& {\left[K_{3, r}(\mathbf{W}, \mathbf{V})\right]=\frac{1}{2} \int_{\Omega_{r}}\left[B_{r}^{N L}\left(\mathbf{x}_{r}, \mathbf{W}\right)\right]^{\mathrm{T}}\left[C_{e, r}\left(0, \mathbf{x}_{r}\right)\right]\left[B_{r}^{N L}\left(\mathbf{x}_{r}, \mathbf{V}\right)\right] d \mathbf{x}_{r},} \\
& {\left[M_{r}\right]=\int_{\Omega_{r}} \rho_{r}\left[N_{r}\left(\mathbf{x}_{r}\right)\right]^{\mathrm{T}}\left[N_{r}\left(\mathbf{x}_{r}\right)\right] d \mathbf{x}_{r},} \\
& {\left[C_{r}\right]=2 \int_{\Omega_{r}} \rho_{r}\left[N_{r}\left(\mathbf{x}_{r}\right)\right]^{\mathrm{T}}[R]\left[N_{r}\left(\mathbf{x}_{r}\right)\right] d \mathbf{x}_{r},} \\
& {\left[G_{r}(t)\right]=\int_{\Omega_{r}}\left[B_{r}\left(\mathbf{x}_{r}\right)\right]^{\mathrm{T}}\left[\dot{C}_{e, r}\left(t, \mathbf{x}_{r}\right)\right]\left[B_{r}\left(\mathbf{x}_{r}\right)\right] d \mathbf{x}_{r},} \\
& {\left[G_{2, r}(t, \mathbf{V})\right]=\int_{\Omega_{r}}\left[B_{r}\left(\mathbf{x}_{r}\right)\right]^{\mathrm{T}}\left[\dot{C}_{e, r}\left(t, \mathbf{x}_{r}\right)\right]\left[B_{r}^{N L}\left(\mathbf{x}_{r}, \mathbf{V}\right)\right] d \mathbf{x}_{r},} \\
& {\left[G_{3, r}(t, \mathbf{W}, \mathbf{V})\right]=\frac{1}{2} \int_{\Omega_{r}}\left[B_{r}^{N L}\left(\mathbf{x}_{r}, \mathbf{W}\right)\right]^{\mathrm{T}}\left[\dot{C}_{e, r}\left(t, \mathbf{x}_{r}\right)\right]\left[B_{r}^{N L}\left(\mathbf{x}_{r}, \mathbf{V}\right)\right] d \mathbf{x}_{r},} \\
& {\left[\widehat{K}_{g, r}(t)\right]=\int_{\Omega_{r}}\left[B_{r}\left(\mathbf{x}_{r}\right)\right]^{\mathrm{T}}\left[\widehat{C}_{g, r}\left(t, \mathbf{x}_{r}\right)\right]\left[B_{r}\left(\mathbf{x}_{r}\right)\right] d \mathbf{x}_{r},} \\
& {\left[\widehat{K}_{e, r}(t)\right]=\left[K_{2, r}\left(\mathbf{U}_{t}^{\text {tran }}\right)\right]+\left[K_{2, r}\left(\mathbf{U}_{t}^{\text {tran }}\right)\right]^{\mathrm{T}}+2\left[K_{3, r}\left(\mathbf{U}_{t}^{\text {tran }}, \mathbf{U}_{t}^{\text {tran }}\right)\right],} \\
& {\left[\widehat{K}_{2, r}(t, \mathbf{V})\right]=\left[K_{3, r}\left(\mathbf{U}_{t}^{\text {tran }}, \mathbf{V}\right)\right],} \\
& {\left[\widehat{G}_{r}(\tau, t)\right]=\left[G_{2, r}\left(\tau, \mathbf{U}_{t}^{\text {tran }}\right)\right]^{\mathrm{T}}+\left[G_{2, r}\left(\tau, \mathbf{U}_{t-\tau}^{\text {tran }}\right)+2\left[G_{3, r}\left(\tau, \mathbf{U}_{t}^{\text {tran }}, \mathbf{U}_{t-\tau}^{\text {tran }}\right)\right],\right.} \\
& {\left[\widehat{B}_{2, r}(\tau, t, \mathbf{V})\right]=2\left[G_{3, r}\left(\tau, \mathbf{V}, \mathbf{U}_{t-\tau}^{\text {tran }}\right)\right],} \\
& {\left[\widehat{G}_{2, r}(\tau, t, \mathbf{V})\right]=\left[G_{3, r}\left(\tau, \mathbf{U}_{t}^{\text {tran }}, \mathbf{V}\right)\right] .} \\
& \widehat{\mathbf{L}}_{a, h}(\tau, t, \mathbf{U})=\widehat{l}_{a, r}\left(\tau, t,\left[N_{r}\right] \mathbf{U}\right), \\
& \mathbf{L}_{r, h}(t)=\int_{\Omega_{r}} \rho_{r}\left[N_{r}\left(\mathbf{x}_{r}\right)\right]^{\mathrm{T}} \mathbf{f}_{r}^{d y n}\left(t, \mathbf{x}_{r}\right) d \mathbf{x}_{r}, \\
& \widehat{\mathbf{L}}_{r, h}(t)=\left[M_{r}\right] \ddot{\mathbf{U}}_{t}^{\text {tran }}+\left[C_{r}\right] \dot{\mathbf{U}}_{t}^{\text {tran }} \\
& +\left\{\frac{1}{2}\left[K_{2, r}\left(\mathbf{U}^{\text {tran }}(t-\tau)\right)\right]+\left[K_{2, r}\left(\mathbf{U}_{t}^{\text {tran }}\right)\right]^{\mathrm{T}}+\left[K_{3, r}\left(\mathbf{U}_{t}^{\text {tran }}, \mathbf{U}_{t}^{\text {tran }}\right)\right]\right\} \mathbf{U}_{t}^{\text {tran }} \\
& +\int_{0}^{+\infty}\left\{\left[G_{r}(\tau)\right]+\left[G_{2, r}\left(\tau, \mathbf{U}_{t}^{\text {tran }}\right)\right]^{\mathrm{T}}+\frac{1}{2}\left[G_{2, r}\left(\tau, \mathbf{U}_{t-\tau}^{\text {tran }}\right)\right]\right. \\
& \left.+\left[G_{3, r}\left(\tau, \mathbf{U}_{t}^{\operatorname{tran}}, \mathbf{U}_{t-\tau}^{\operatorname{tran}}\right)\right]\right\} \mathbf{U}_{t-\tau}^{\operatorname{tran}} d \tau
\end{aligned}
$$


where, for all $\mathbf{U}$ in $\mathbb{R}^{N_{h}}$, we have

$$
\begin{gathered}
{\left[B_{r}\left(\mathbf{x}_{r}\right)\right]_{d_{i j} p}=\frac{\partial\left[N_{r}\left(\mathbf{x}_{r}\right)\right]_{i p}}{\partial\left\{\mathbf{x}_{r}\right\}_{j}},} \\
{\left[B_{r}^{N L}\left(\mathbf{x}_{r}, \mathbf{U}\right)\right]_{d_{i j} p}=\frac{\partial\left[N_{r}\left(\mathbf{x}_{r}\right)\right]_{k p}}{\partial\left\{\mathbf{x}_{r}\right\}_{j}} \frac{\partial\left[N_{r}\left(\mathbf{x}_{r}\right)\right]_{k q}}{\partial\left\{\mathbf{x}_{r}\right\}_{i}}\{\mathbf{U}\}_{q},} \\
{\left[C_{e, r}\left(\tau, \mathbf{x}_{r}\right)\right]_{d_{i j} d_{k h}}=\left\{\mathbb{G}_{r}\left(\tau, \mathbf{x}_{r}\right)\right\}_{i j k h},} \\
{\left[C_{g, r}\left(\mathbf{x}_{r}\right)\right]_{d_{a i} d_{b j}}=\delta_{a b}\left\{\sigma_{r}\left(\mathbf{x}_{r}\right)\right\}_{i j},} \\
{\left[\widehat{C}_{g, r}\left(t, \mathbf{x}_{r}\right)\right]_{d_{a i} d_{b j}}=\frac{1}{2} \delta_{a b}\left\{\left[C_{e, r}\left(0, \mathbf{x}_{r}\right)\right]\left[B_{r}^{N L}\left(\mathbf{x}_{r}, \mathbf{U}_{t}^{\text {tran }}\right)\right] \mathbf{U}_{t}^{\text {tran }}\right\}_{d_{i j}}} \\
+\delta_{a b}\left\{\left[C_{e, r}\left(0, \mathbf{x}_{r}\right)\right]\left[B_{r}\left(\mathbf{x}_{r}\right)\right] \mathbf{U}_{t}^{\text {tran }}\right\}_{d_{i j}} \\
+\frac{\delta_{a b}}{2} \int_{0}^{+\infty}\left\{\left[\dot{C}_{e, r}\left(\tau, \mathbf{x}_{r}\right)\right]\left[B_{r}^{N L}\left(\mathbf{x}_{r}, \mathbf{U}_{t-\tau}^{\text {tran }}\right)\right] \mathbf{U}_{t-\tau}^{\text {tran }}\right\}_{d_{i j}} d \tau \\
+\int_{0}^{+\infty} \delta_{a b}\left\{\left[\dot{C}_{e, r}\left(\tau, \mathbf{x}_{r}\right)\right]\left[B_{r}\left(\mathbf{x}_{r}\right)\right] \mathbf{U}_{t-\tau}^{\text {tran }}\right\}_{d_{i j}} d \tau
\end{gathered}
$$

\section{Appendix D. Definition of tensors introduced in the sensitivity analysis}

$$
\begin{aligned}
& \left\{\mathbb{T}_{1} \circ \mathbf{X}_{0}^{r e f}\right\}_{a b c d}=-\frac{1}{\operatorname{det} \mathbb{F}_{0}^{r e f}}\left\{\mathbb{F}_{0}^{r e f}\right\}_{a d}\left\{\mathbb{F}_{0}^{r e f}\right\}_{b l}\left\{\mathbb{E}_{0}^{r e f}\right\}_{p q}\left\{\mathbb{A}_{0}\right\}_{c l p q} \\
& -\frac{1}{\operatorname{det} \mathbb{F}_{0}^{r e f}}\left\{\mathbb{F}_{0}^{r e f}\right\}_{a k}\left\{\mathbb{F}_{0}^{r e f}\right\}_{b d}\left\{\mathbb{E}_{0}^{r e f}\right\}_{p q}\left\{\mathbb{A}_{0}\right\}_{k c p q} \\
& -\frac{1}{\operatorname{det} \mathbb{F}_{0}^{r e f}}\left\{\mathbb{F}_{0}^{r e f}\right\}_{a k}\left\{\mathbb{F}_{0}^{r e f}\right\}_{b l}\left\{\mathbb{E}_{0}^{r e f}\right\}_{q d}\left\{\mathbb{A}_{0}\right\}_{k l c q} \\
& -\frac{1}{\operatorname{det} \mathbb{F}_{0}^{r e f}}\left\{\mathbb{F}_{0}^{r e f}\right\}_{a k}\left\{\mathbb{F}_{0}^{r e f}\right\}_{b l}\left\{\mathbb{E}_{0}^{r e f}\right\}_{p d}\left\{\mathbb{A}_{0}\right\}_{k l p c} \\
& -\frac{1}{\operatorname{det} \mathbb{F}_{0}^{r e f}}\left\{\mathbb{F}_{0}^{r e f}\right\}_{a k}\left\{\mathbb{F}_{0}^{r e f}\right\}_{b l}\left\{\mathbb{A}_{0}\right\}_{k l d c} \\
& \left\{\mathbb{T}_{2} \circ \mathbf{X}_{0}^{r e f}\right\}_{a b p q i j}=-\frac{1}{\operatorname{det} \mathbb{F}_{0}^{r e f}}\left\{\mathbb{F}_{0}^{r e f}\right\}_{a j}\left\{\mathbb{F}_{0}^{r e f}\right\}_{b l}\left\{\mathbb{F}_{0}^{r e f}\right\}_{p m}\left\{\mathbb{F}_{0}^{r e f}\right\}_{q n}\left\{\mathbb{G}_{0}\right\}_{i l m n} \\
& -\frac{1}{\operatorname{det} \mathbb{F}_{0}^{r e f}}\left\{\mathbb{F}_{0}^{r e f}\right\}_{a k}\left\{\mathbb{F}_{0}^{r e f}\right\}_{b j}\left\{\mathbb{F}_{0}^{r e f}\right\}_{p m}\left\{\mathbb{F}_{0}^{r e f}\right\}_{q n}\left\{\mathbb{G}_{0}\right\}_{k i m n} \\
& -\frac{1}{\operatorname{det} \mathbb{F}_{0}^{r e f}}\left\{\mathbb{F}_{0}^{r e f}\right\}_{a k}\left\{\mathbb{F}_{0}^{r e f}\right\}_{b l}\left\{\mathbb{F}_{0}^{r e f}\right\}_{p j}\left\{\mathbb{F}_{0}^{r e f}\right\}_{q n}\left\{\mathbb{G}_{0}\right\}_{k l i n} \\
& -\frac{1}{\operatorname{det} \mathbb{F}_{0}^{r e f}}\left\{\mathbb{F}_{0}^{r e f}\right\}_{a k}\left\{\mathbb{F}_{0}^{r e f}\right\}_{b l}\left\{\mathbb{F}_{0}^{r e f}\right\}_{p m}\left\{\mathbb{F}_{0}^{r e f}\right\}_{q j}\left\{\mathbb{G}_{0}\right\}_{k l m i} \\
& \left\{\mathbb{K}_{1} \circ \mathbf{X}_{0}^{r e f}\right\}_{a b c d p q}=\frac{1}{\operatorname{det} \mathbb{F}_{0}^{r e f}}\left\{\mathbb{F}_{0}^{r e f}\right\}_{a q}\left\{\mathbb{F}_{0}^{r e f}\right\}_{b p}\left\{\mathbb{E}_{0}^{r e f}\right\}_{d c}, \\
& \left\{\mathbb{K}_{2} \circ \mathbf{X}_{0}^{r e f}\right\}_{\text {abpqijkh }}=\frac{1}{\operatorname{det} \mathbb{F}_{0}^{r e f}}\left\{\mathbb{F}_{0}^{r e f}\right\}_{a h}\left\{\mathbb{F}_{0}^{r e f}\right\}_{b k}\left\{\mathbb{F}_{0}^{r e f}\right\}_{p j}\left\{\mathbb{F}_{0}^{r e f}\right\}_{q i} .
\end{aligned}
$$


It is straightfoward to show that

$$
\begin{aligned}
& \lim _{\tau \rightarrow+\infty}\left|\left\{\mathbb{K}_{2}\left(\mathbf{X}_{0}^{r e f}\left(\mathbf{x}_{0}\right)\right):: \Delta \mathbb{G}_{\overline{0}}\left(\tau, \mathbf{X}_{0 \overline{0}}\left(\mathbf{x}_{0}\right)\right)\right\}_{a b p q}\right|<+\infty, \\
& \left.\lim _{\tau \rightarrow+\infty} \mid \mathbb{T}_{2}\left(\tau, \mathbf{X}_{0}^{r e f}\left(\mathbf{x}_{0}\right)\right): \frac{\partial \mathbf{u}_{0 \overline{0}}}{\partial \mathbf{x}_{0}}\left(\mathbf{x}_{0}\right)\right\}_{a b p q} \mid<+\infty, \\
& \left\{\mathbb{K}_{1}:: \Delta \mathbb{A}_{\overline{0}}\right\}_{i j}=\left\{\mathbb{K}_{1}:: \Delta \mathbb{A}_{\overline{0}}\right\}_{j i}, \\
& \left\{\mathbb{T}_{1}: \frac{\partial \mathbf{u}_{0 \overline{0}}}{\partial \mathbf{x}_{0}}\right\}_{i j}=\left\{\mathbb{T}_{1}: \frac{\partial \mathbf{u}_{0 \overline{0}}}{\partial \mathbf{x}_{0}}\right\}_{j i}, \\
& \left\{\mathbb{K}_{2}:: \Delta \mathbb{G}_{\overline{0}}\right\}_{i j k h}=\left\{\mathbb{K}_{2}:: \Delta \mathbb{G}_{\overline{0}}\right\}_{k h i j}=\left\{\mathbb{K}_{2}:: \Delta \mathbb{G}_{\overline{0}}\right\}_{j i k h}, \\
& \left\{\mathbb{T}_{2}: \frac{\partial \mathbf{u}_{0 \overline{0}}}{\partial \mathbf{x}_{0}}\right\}_{i j k h}=\left\{\mathbb{T}_{2}: \frac{\partial \mathbf{u}_{0 \overline{0}}}{\partial \mathbf{x}_{0}}\right\}_{k h i j}=\left\{\mathbb{T}_{2}: \frac{\partial \mathbf{u}_{0 \overline{0}}}{\partial \mathbf{x}_{0}}\right\}_{j i k h},
\end{aligned}
$$

where, in order to simplify notations we note

$$
\begin{aligned}
& \mathbb{K}_{1}:: \Delta \mathbb{A}_{\overline{0}} \equiv \mathbb{K}_{1}\left(\mathbf{X}_{0}^{r e f}\left(\mathbf{x}_{0}\right)\right):: \Delta \mathbb{A}_{\overline{0}}\left(\mathbf{X}_{0 \overline{0}}\left(\mathbf{x}_{0}\right)\right), \\
& \mathbb{T}_{1}: \frac{\partial \mathbf{u}_{0 \overline{0}}}{\partial \mathbf{x}_{0}} \equiv \mathbb{T}_{1}\left(\mathbf{X}_{0}^{r e f}\left(\mathbf{x}_{0}\right)\right): \frac{\partial \mathbf{u}_{0 \overline{0}}}{\partial \mathbf{x}_{0}}\left(\mathbf{x}_{0}\right), \\
& \mathbb{K}_{2}:: \Delta \mathbb{G}_{\overline{0}} \equiv \mathbb{K}_{2}\left(\mathbf{X}_{0}^{r e f}\left(\mathbf{x}_{0}\right)\right):: \Delta \mathbb{G}_{\overline{0}}\left(\tau, \mathbf{X}_{0 \overline{0}}\left(\mathbf{x}_{0}\right)\right), \\
& \mathbb{T}_{2}: \frac{\partial \mathbf{u}_{0 \overline{0}}}{\partial \mathbf{x}_{0}} \equiv \mathbb{T}_{2}\left(\tau, \mathbf{X}_{0}^{r e f}\left(\mathbf{x}_{0}\right)\right): \frac{\partial \mathbf{u}_{0 \overline{0}}}{\partial \mathbf{x}_{0}}\left(\mathbf{x}_{0}\right) .
\end{aligned}
$$

From Eqs. (143) to (156), we deduce the symmetric properties of $\Delta \sigma_{r}$ and $\Delta \mathbb{G}_{r}$

$$
\begin{aligned}
& \left\{\Delta \sigma_{r}\left(\mathbf{x}_{r}\right)\right\}_{i j}=\left\{\Delta \sigma_{r}\left(\mathbf{x}_{r}\right)\right\}_{j i}, \\
& \left\{\Delta \mathbb{G}_{r}\left(t, \mathbf{x}_{r}\right)\right\}_{i j k h}=\left\{\Delta \mathbb{G}_{r}\left(t, \mathbf{x}_{r}\right)\right\}_{k h i j}=\left\{\Delta \mathbb{G}_{r}\left(t, \mathbf{x}_{r}\right)\right\}_{j i k h} .
\end{aligned}
$$

\section{Appendix E. Definition of the multilinear forms for the perturbed problem}

$$
\begin{gathered}
\Delta k_{g, r}(\mathbf{u}, \mathbf{v})=\int_{\Omega_{r}} \Delta \sigma_{r}:\left\{\frac{\partial \mathbf{u}^{\mathrm{T}}}{\partial \mathbf{x}_{r}} \frac{\partial \mathbf{v}}{\partial \mathbf{x}_{r}}\right\} d \mathbf{x}_{r}, \\
\Delta k_{e, r}(\mathbf{u}, \mathbf{v})=\int_{\Omega_{r}}\left\{\Delta \mathbb{G}_{r}\left(0, \mathbf{x}_{r}\right): \frac{\partial \mathbf{u}}{\partial \mathbf{x}_{r}}\right\}: \frac{\partial \mathbf{v}}{\partial \mathbf{x}_{r}} d \mathbf{x}_{r}, \\
\Delta k_{c, r}(\mathbf{u}, \mathbf{v})=\int_{\Omega_{r}} \Delta \rho_{r}\left\{[R]^{2} \mathbf{u}\right\} \cdot \mathbf{v} d \mathbf{x}_{r}, \\
\Delta g_{r}(t, \mathbf{u}, \mathbf{v})=\int_{\Omega_{r}}\left\{\Delta \dot{\mathbb{G}}_{r}\left(t, \mathbf{x}_{r}\right): \frac{\partial \mathbf{u}}{\partial \mathbf{x}_{r}}\right\}: \frac{\partial \mathbf{v}}{\partial \mathbf{x}_{r}} d \mathbf{x}_{r}, \\
\Delta c_{r}(\mathbf{u}, \mathbf{v})=2 \int_{\Omega_{r}} \Delta \rho_{r}\{[R] \mathbf{u}\} \cdot \mathbf{v} d \mathbf{x}_{r}, \\
\Delta m_{r}(\mathbf{u}, \mathbf{v})=\int_{\Omega_{r}} \Delta \rho_{r} \mathbf{u} \cdot \mathbf{v} d \mathbf{x}_{r},
\end{gathered}
$$




$$
\Delta g_{2, r}(t, \mathbf{u}, \mathbf{v}, \mathbf{w})=\int_{\Omega_{r}}\left\{\Delta \dot{\mathbb{G}}_{r}\left(t, \mathbf{x}_{r}\right):\left(\frac{\partial \mathbf{u}^{\mathrm{T}}}{\partial \mathbf{x}_{r}} \frac{\partial \mathbf{v}}{\partial \mathbf{x}_{r}}\right)\right\}: \frac{\partial \mathbf{w}}{\partial \mathbf{x}_{r}} d \mathbf{x}_{r}
$$

$\Delta g_{3, r}(t, \mathbf{u}, \mathbf{v}, \mathbf{w}, \mathbf{r})=\frac{1}{2} \int_{\Omega_{r}}\left\{\Delta \dot{\mathbb{G}}_{r}\left(t, \mathbf{x}_{r}\right):\left(\frac{\partial \mathbf{u}^{\mathrm{T}}}{\partial \mathbf{x}_{r}} \frac{\partial \mathbf{v}}{\partial \mathbf{x}_{r}}\right)\right\}:\left\{\frac{\partial \mathbf{w}^{\mathrm{T}}}{\partial \mathbf{x}_{r}} \frac{\partial \mathbf{r}}{\partial \mathbf{x}_{r}}\right\} d \mathbf{x}_{r}$,

$$
\Delta k_{2, r}(\mathbf{u}, \mathbf{v}, \mathbf{w})=\int_{\Omega_{r}}\left\{\Delta \mathbb{G}_{r}\left(0, \mathbf{x}_{r}\right):\left(\frac{\partial \mathbf{u}^{\mathrm{T}}}{\partial \mathbf{x}_{r}} \frac{\partial \mathbf{v}}{\partial \mathbf{x}_{r}}\right)\right\}: \frac{\partial \mathbf{w}}{\partial \mathbf{x}_{r}} d \mathbf{x}_{r},
$$

$$
\Delta k_{3, r}(\mathbf{u}, \mathbf{v}, \mathbf{w}, \mathbf{r})=\frac{1}{2} \int_{\Omega_{r}}\left\{\Delta \mathbb{G}_{r}\left(0, \mathbf{x}_{r}\right):\left(\frac{\partial \mathbf{u}^{\mathrm{T}}}{\partial \mathbf{x}_{r}} \frac{\partial \mathbf{v}}{\partial \mathbf{x}_{r}}\right)\right\}:\left\{\frac{\partial \mathbf{w}^{\mathrm{T}}}{\partial \mathbf{x}_{r}} \frac{\partial \mathbf{r}}{\partial \mathbf{x}_{r}}\right\} d \mathbf{x}_{r},
$$

$$
\Delta l_{a, r}(\mathbf{v})=-\int_{\Sigma_{r}}\left\{\Delta \sigma_{r} \mathbf{n}_{r}\right\} \cdot \mathbf{v} d \mathbf{x}_{r}, \quad \Delta l_{r}(t, \mathbf{v})=\int_{\Omega_{r}} \Delta \rho_{r} \mathbf{f}_{r}^{d y n^{\prime}}\left(t, \mathbf{x}_{r}\right) \cdot \mathbf{v} d \mathbf{x}_{r},
$$

$$
\begin{aligned}
& \Delta \widehat{k}_{g, r}(t, \mathbf{u}, \mathbf{v})=\int_{\Omega_{r}} \int_{0}^{+\infty}\left\{\Delta \dot{\mathbb{G}}_{r}\left(\tau, \mathbf{x}_{r}\right): \mathbb{E}_{r}^{\text {tran }}\left(t-\tau, \mathbf{x}_{r}\right)\right\}:\left\{\frac{\partial \mathbf{u}^{\mathrm{T}}}{\partial \mathbf{x}_{r}} \frac{\partial \mathbf{v}}{\partial \mathbf{x}_{r}}\right\} d \mathbf{x}_{r} d \tau \\
&+\int_{\Omega_{r}}\left\{\Delta \mathbb{G}_{r}\left(0, \mathbf{x}_{r}\right): \mathbb{E}_{r}^{\text {tran }}\left(t, \mathbf{x}_{r}\right)\right\}:\left\{\frac{\partial \mathbf{u}^{\mathrm{T}}}{\partial \mathbf{x}_{r}} \frac{\partial \mathbf{v}}{\partial \mathbf{x}_{r}}\right\} d \mathbf{x}_{r} \\
& \Delta \widehat{k}_{e, r}(t, \mathbf{u}, \mathbf{v})=\Delta k_{2, r}\left(\mathbf{u}, \mathbf{u}_{r}^{\text {tran }}(t), \mathbf{v}\right)+\Delta k_{2, r}\left(\mathbf{v}, \mathbf{u}_{r}^{\text {tran }}(t), \mathbf{u}\right) \\
&+2 \Delta k_{3, r}\left(\mathbf{u}, \mathbf{u}_{r}^{\text {tran }}(t), \mathbf{u}_{r}^{\text {tran }}(t), \mathbf{v}\right)
\end{aligned}
$$

$\Delta \widehat{g}_{r}(\tau, t, \mathbf{u}, \mathbf{v})=\Delta g_{2, r}\left(\tau, \mathbf{v}, \mathbf{u}_{r}^{\operatorname{tran}}(t), \mathbf{u}\right)+\Delta g_{2, r}\left(\tau, \mathbf{u}, \mathbf{u}_{r}^{\operatorname{tran}}(t-\tau), \mathbf{v}\right)$

$$
+2 \Delta g_{3, r}\left(\tau, \mathbf{u}_{r}^{\operatorname{tran}}(t-\tau), \mathbf{u}, \mathbf{u}_{r}^{\operatorname{tran}}(t), \mathbf{v}\right),
$$

$$
\Delta \widehat{b}_{2, r}(\tau, t, \mathbf{u}, \mathbf{v}, \mathbf{w})=2 \Delta g_{3, r}\left(\tau, \mathbf{u}_{r}^{\operatorname{tran}}(t-\tau), \mathbf{u}, \mathbf{v}, \mathbf{w}\right),
$$$$
\Delta \widehat{k}_{2, r}(t, \mathbf{u}, \mathbf{v}, \mathbf{w})=\Delta k_{3, r}\left(\mathbf{u}, \mathbf{v}, \mathbf{u}_{r}^{\operatorname{tran}}(t), \mathbf{w}\right)
$$$$
\Delta \widehat{g}_{2, r}(\tau, t, \mathbf{u}, \mathbf{v}, \mathbf{w})=\Delta g_{3, r}\left(\tau, \mathbf{u}, \mathbf{v}, \mathbf{u}_{r}^{\operatorname{tran}}(t), \mathbf{w}\right),
$$

$$
\begin{aligned}
\Delta \widehat{l}_{r}(t, \mathbf{v}) & =\Delta m_{r}\left(\ddot{\mathbf{u}}_{r}^{\text {tran }}, \mathbf{v}\right)+\Delta c_{r}\left(\dot{\mathbf{u}}_{r}^{\text {tran }}, \mathbf{v}\right) \\
& +\Delta k_{g, r}\left(\mathbf{u}_{r}^{\text {tran }}, \mathbf{v}\right)+\Delta k_{c, r}\left(\mathbf{u}_{r}^{\text {tran }}, \mathbf{v}\right)+\Delta k_{e, r}\left(\mathbf{u}_{r}^{\text {tran }}, \mathbf{v}\right)+\Delta k_{2, r}\left(\mathbf{v}, \mathbf{u}_{r}^{\text {tran }}, \mathbf{u}_{r}^{\text {tran }}\right) \\
& +\frac{1}{2} \Delta k_{2, r}\left(\mathbf{u}_{r}^{\text {tran }}, \mathbf{u}_{r}^{\text {tran }}, \mathbf{v}\right)+\Delta k_{3, r}\left(\mathbf{u}_{r}^{\text {tran }}, \mathbf{u}_{r}^{\text {tran }}, \mathbf{u}_{r}^{\text {tran }}, \mathbf{v}\right) \\
& +\int_{0}^{+\infty} \Delta g_{r}\left(\tau, \mathbf{u}_{r}^{\text {tran }}(t-\tau), \mathbf{v}\right) d \tau+\int_{0}^{+\infty} \Delta g_{2, r}\left(\tau, \mathbf{v}, \mathbf{u}_{r}^{\text {tran }}(t), \mathbf{u}_{r}^{\text {tran }}(t-\tau)\right) d \tau \\
& +\frac{1}{2} \int_{0}^{+\infty} \Delta g_{2, r}\left(\tau, \mathbf{u}_{r}^{\text {tran }}(t-\tau), \mathbf{u}_{r}^{\text {tran }}(t-\tau), \mathbf{v}\right) d \tau \\
& +\int_{0}^{+\infty} \Delta g_{3, r}\left(\tau, \mathbf{u}_{r}^{\text {tran }}(t-\tau), \mathbf{u}_{r}^{\text {tran }}(t-\tau), \mathbf{u}_{r}^{\text {tran }}(t), \mathbf{v}\right) d \tau,
\end{aligned}
$$




$$
\Delta k_{3, r}(\mathbf{u}, \mathbf{v}, \mathbf{w}, \mathbf{r})=\Delta k_{3, r}(\mathbf{u}, \mathbf{v}, \mathbf{r}, \mathbf{w})=\Delta k_{3, r}(\mathbf{w}, \mathbf{r}, \mathbf{u}, \mathbf{v})
$$

From Eq. (158), for all w fixed in $\mathcal{C}_{a d}\left(\Omega_{r}\right)$, we deduce that, $\Delta \widehat{k}_{g, r}(t, \cdot), \Delta \widehat{k}_{e, r}(t, \cdot),(\mathbf{u}, \mathbf{v}) \mapsto$ $\Delta \widehat{b}_{2, r}(\tau, t, \mathbf{w}, \mathbf{u}, \mathbf{v}),(\mathbf{u}, \mathbf{v}) \mapsto \Delta \widehat{g}_{2, r}(\tau, t, \mathbf{u}, \mathbf{v}, \mathbf{w})$ and $(\mathbf{u}, \mathbf{v}) \mapsto \Delta \widehat{k}_{2, r}(t, \mathbf{u}, \mathbf{v}, \mathbf{w})$ are symmetric bilinear forms.

Appendix F. Definition of the multilinear operators introduced by the use of the formal perturbation method

$\mathbf{A}_{r}\left(t, \mathbf{u}_{r}^{h o m \prime}\right)=\left\{\mathbf{K}_{e, r}+\mathbf{K}_{g, r}+\mathbf{K}_{c, r}+\frac{1}{2} \mathbf{K}_{2, r}\left(\mathbf{u}_{r}^{h o m \prime}(t)\right)+{ }^{\mathrm{t}} \mathbf{K}_{2, r}\left(\mathbf{u}_{r}^{h o m \prime}(t)\right)\right.$

$$
\left.+\mathbf{K}_{3, r}\left(\mathbf{u}_{r}^{\text {hom' }}(t), \mathbf{u}_{r}^{h o m \prime}(t)\right)\right\} \mathbf{u}_{r}^{h o m \prime}(t)
$$$$
+\int_{0}^{+\infty}\left\{\frac{1}{2} \mathbf{G}_{2, r}\left(\tau, \mathbf{u}_{r}^{h o m \prime}(t-\tau)\right)+{ }^{\mathrm{t}} \mathbf{G}_{2, r}\left(\tau, \mathbf{u}_{r}^{\text {hom' }}(t)\right)\right.
$$$$
\left.+\mathbf{G}_{3, r}\left(\tau, \mathbf{u}_{r}^{h o m \prime}(t), \mathbf{u}_{r}^{h o m \prime}(t-\tau)\right)+\mathbf{G}_{r}(\tau)\right\} \mathbf{u}_{r}^{h o m \prime}(t-\tau) d \tau
$$

$$
-\int_{0}^{\infty} \widehat{\mathbf{L}}_{a, r}\left(\tau, t, \mathbf{u}_{r}^{\text {hom' }}(t-\tau)\right) d \tau-\mathbf{L}_{r}(t)
$$

$\widehat{\mathbf{A}}_{r}\left(t, \mathbf{u}_{r}^{h o m \prime}\right)=\left\{\widehat{\mathbf{K}}_{g, r}(t)+\widehat{\mathbf{K}}_{e, r}(t)+\widehat{\mathbf{K}}_{2, r}\left(t, \mathbf{u}_{r}^{h o m \prime}(t)\right)+2{ }^{t} \widehat{\mathbf{K}}_{2, r}\left(t, \mathbf{u}_{r}^{h o m \prime}(t)\right)\right\} \mathbf{u}_{r}^{h o m \prime}(t)$

$$
\begin{aligned}
& +\int_{0}^{+\infty}\left\{\widehat{\mathbf{B}}_{2, r}\left(\tau, t, \mathbf{u}_{r}^{h o m \prime}(t)\right)+\widehat{\mathbf{G}}_{2, r}\left(\tau, t, \mathbf{u}_{r}^{h o m \prime}(t-\tau)+\widehat{\mathbf{G}}_{r}(\tau, t)\right\} \mathbf{u}_{r}^{h o m \prime}(t-\tau) d \tau\right. \\
& +\widehat{\mathbf{L}}_{r}(t)
\end{aligned}
$$

$$
\begin{aligned}
& \Delta \mathbf{A}_{r}\left(t, \mathbf{u}_{r}^{h o m \prime}\right)=\left\{\Delta \mathbf{K}_{e, r}+\Delta \mathbf{K}_{c, r}+\Delta \mathbf{K}_{g, r}+\frac{1}{2} \Delta \mathbf{K}_{2, r}\left(\mathbf{u}_{r}^{h o m \prime}(t)\right)+{ }^{\mathrm{t}} \Delta \mathbf{K}_{2, r}\left(\mathbf{u}_{r}^{\text {hom }}(t)\right)\right. \\
& \left.+\Delta \mathbf{K}_{3, r}\left(\mathbf{u}_{r}^{h o m \prime}(t), \mathbf{u}_{r}^{h o m \prime}(t)\right)\right\} \mathbf{u}_{r}^{h o m \prime}(t) \\
& +\int_{0}^{+\infty}\left\{\frac{1}{2} \Delta \mathbf{G}_{2, r}\left(\tau, \mathbf{u}_{r}^{\text {hom' }}(t-\tau)\right)+{ }^{\mathrm{t}} \Delta \mathbf{G}_{2, r}\left(\tau, \mathbf{u}_{r}^{\text {hom' }}(t)\right)\right. \\
& \left.+\Delta \mathbf{G}_{3, r}\left(\tau, \mathbf{u}_{r}^{h o m \prime}(t), \mathbf{u}_{r}^{h o m \prime}(t-\tau)\right)+\Delta \mathbf{G}_{r}(\tau)\right\} \mathbf{u}_{r}^{\text {hom' }}(t-\tau) d \tau \\
& -\Delta \widehat{\mathbf{L}}_{a, r}-\Delta \mathbf{L}_{r}(t),
\end{aligned}
$$

$$
\begin{aligned}
& \Delta \widehat{\mathbf{A}}_{r}\left(t, \mathbf{u}_{r}^{h o m \prime}\right)=\left\{\Delta \widehat{\mathbf{K}}_{g, r}(t)+\Delta \widehat{\mathbf{K}}_{e, r}(t)+\Delta \widehat{\mathbf{K}}_{2, r}\left(t, \mathbf{u}_{r}^{h o m \prime}(t)\right)\right. \\
& \left.+2{ }^{\mathrm{t}} \Delta \widehat{\mathbf{K}}_{2, r}\left(t, \mathbf{u}_{r}^{\text {hom' }}(t)\right)\right\} \mathbf{u}_{r}^{\text {hom' }}(t) \\
& +\int_{0}^{+\infty}\left\{\Delta \widehat{\mathbf{G}}_{2, r}\left(\tau, t, \mathbf{u}_{r}^{h o m \prime}(t-\tau)\right)+\Delta \widehat{\mathbf{G}}_{r}(\tau, t)\right. \\
& \left.+\Delta \widehat{\mathbf{B}}_{2, r}\left(\tau, t, \mathbf{u}_{r}^{h o m \prime}(t)\right)\right\} \mathbf{u}_{r}^{h o m \prime}(t-\tau) d \tau+\Delta \widehat{\mathbf{L}}_{r}(t), \\
& \mathbf{K}_{g, r}^{0}(t) \mathbf{u}=\left\{\mathbf{K}_{3, r}\left(\mathbf{u}, \mathbf{u}_{r}^{0}(t)\right)+{ }^{\mathrm{t}} \mathbf{K}_{2, r}(\mathbf{u})\right\} \mathbf{u}_{r}^{0}(t)+\int_{0}^{+\infty}\left\{\mathbf{G}_{3, r}\left(\tau, \mathbf{u}, \mathbf{u}_{r}^{0}(t-\tau)\right)\right.
\end{aligned}
$$




$$
\left.+{ }^{\mathrm{t}} \mathbf{G}_{2, r}(\tau, \mathbf{u})\right\} \mathbf{u}_{r}^{0}(t-\tau) d \tau
$$

$$
\begin{aligned}
& \widehat{\mathbf{K}}_{g, r}^{0}(t) \mathbf{u}=2{ }^{t} \widehat{\mathbf{K}}_{2, r}(t, \mathbf{u}) \mathbf{u}_{r}^{0}(t)+\int_{0}^{+\infty} \widehat{\mathbf{B}}_{2, r}(\tau, t, \mathbf{u}) \mathbf{u}_{r}^{0}(t-\tau) d \tau, \\
& \mathbf{K}_{r}^{0}(t) \mathbf{u}=\left\{\mathbf{K}_{g, r}^{0}(t)+\mathbf{K}_{2, r}\left(\mathbf{u}_{r}^{0}(t)\right)+{ }^{\mathrm{t}} \mathbf{K}_{2, r}\left(\mathbf{u}_{r}^{0}(t)\right)+2 \mathbf{K}_{3, r}\left(\mathbf{u}_{r}^{0}(t), \mathbf{u}_{r}^{0}(t)\right)\right\} \mathbf{u}, \\
& \widehat{\mathbf{K}}_{r}^{0}(t) \mathbf{u}=\left\{\widehat{\mathbf{K}}_{e, r}(t)+\widehat{\mathbf{K}}_{g, r}(t)+2 \widehat{\mathbf{K}}_{2, r}\left(t, \mathbf{u}_{r}^{0}(t)\right)+2 \widehat{\mathbf{K}}_{2, r}\left(t, \mathbf{u}_{r}^{0}(t)\right)+\widehat{\mathbf{K}}_{g, r}^{0}(t)\right\} \mathbf{u}, \\
& \mathbf{G}_{r}^{0}(\tau, t) \mathbf{u}=\left\{\mathbf{G}_{r}(\tau)+2 \mathbf{G}_{2, r}\left(\tau, \mathbf{u}_{r}^{0}(t-\tau)\right)+2 \mathbf{G}_{3, r}\left(\tau, \mathbf{u}_{r}^{0}(t), \mathbf{u}_{r}^{0}(t-\tau)\right)\right\} \mathbf{u}, \\
& \widehat{\mathbf{G}}_{r}^{0}(\tau, t) \mathbf{u}=\left\{\widehat{\mathbf{G}}_{r}(\tau, t)+2 \widehat{\mathbf{G}}_{2, r}\left(\tau, t, \mathbf{u}_{r}^{0}(t-\tau)\right)+\widehat{\mathbf{B}}_{2, r}\left(\tau, t, \mathbf{u}_{r}^{0}(t)\right)\right\} \mathbf{u}, \\
& \mathbf{K}_{r}=\mathbf{K}_{e, r}+\mathbf{K}_{g, r}+\mathbf{K}_{c, r} \\
& \mathbf{F}_{r}^{1}(t)=\Delta \mathbf{M}_{r} \ddot{\mathbf{u}}_{r}^{0}(t)+\Delta \mathbf{C}_{r} \dot{\mathbf{u}}_{r}^{0}(t)+\Delta \mathbf{A}_{r}\left(t, \mathbf{u}_{r}^{0}\right), \\
& \widehat{\mathbf{F}}_{r}^{1}(t)=\Delta \widehat{\mathbf{A}}_{r}\left(t, \mathbf{u}_{0}\right), \\
& \mathbf{F}_{r}^{2}(t)=\Delta \mathbf{M}_{r} \ddot{\mathbf{u}}_{r}^{1}(t)+\Delta \mathbf{C}_{r} \dot{\mathbf{u}}_{r}^{1}(t) \\
& +\left\{\frac{1}{2} \mathbf{K}_{2, r}\left(\mathbf{u}_{r}^{1}(t)\right)+{ }^{t} \mathbf{K}_{2, r}\left(\mathbf{u}_{r}^{1}(t)\right)+2 \mathbf{K}_{3, r}\left(\mathbf{u}_{r}^{1}(t), \mathbf{u}_{r}^{0}(t)\right)+\mathbf{K}_{3, r}\left(\mathbf{u}_{r}^{0}(t), \mathbf{u}_{r}^{1}(t)\right)\right. \\
& +\Delta \mathbf{K}_{e, r}+\Delta \mathbf{K}_{g, r}+\Delta \mathbf{K}_{c, r}+\mathbf{K}_{2, r}\left(\mathbf{u}_{r}^{0}(t)\right)+{ }^{\mathrm{t}} \Delta \mathbf{K}_{2, r}\left(\mathbf{u}_{r}^{0}(t)\right) \\
& \left.+2 \Delta \mathbf{K}_{3, r}\left(\mathbf{u}_{r}^{0}(t), \mathbf{u}_{r}^{0}(t)\right)\right\} \mathbf{u}_{r}^{1}(t)+\left\{\Delta \mathbf{K}_{3, r}\left(\mathbf{u}_{r}^{1}(t), \mathbf{u}_{r}^{0}(t)\right)+{ }^{\mathrm{t}} \Delta \mathbf{K}_{2, r}\left(\mathbf{u}_{r}^{1}(t)\right)\right\} \mathbf{u}_{r}^{0}(t) \\
& +\int_{0}^{+\infty}\left\{\frac{1}{2} \mathbf{G}_{2, r}\left(\tau, \mathbf{u}_{r}^{1}(t-\tau)\right)+{ }^{\mathrm{t}} \mathbf{G}_{2, r}\left(\tau, \mathbf{u}_{r}^{1}(t)\right)+2 \mathbf{G}_{3, r}\left(\tau, \mathbf{u}_{r}^{1}(t), \mathbf{u}_{r}^{0}(t-\tau)\right)\right. \\
& +\mathbf{G}_{3, r}\left(\tau, \mathbf{u}_{r}^{0}(t), \mathbf{u}_{r}^{1}(t-\tau)\right)+2 \Delta \mathbf{G}_{2, r}\left(\tau, \mathbf{u}_{r}^{0}(t-\tau)\right) \\
& \left.+2 \Delta \mathbf{G}_{3, r}\left(\tau, \mathbf{u}_{r}^{0}(t), \mathbf{u}_{r}^{0}(t-\tau)\right)+\Delta \mathbf{G}_{r}(\tau)\right\} \mathbf{u}_{r}^{1}(t-\tau) d \tau \\
& +\int_{0}^{+\infty}\left\{{ }^{\mathrm{t}} \Delta \mathbf{G}_{2, r}\left(\tau, \mathbf{u}_{r}^{1}(t)\right)+\Delta \mathbf{G}_{3, r}\left(\tau, \mathbf{u}_{r}^{1}(t), \mathbf{u}_{r}^{0}(t-\tau)\right)\right\} \mathbf{u}_{r}^{0}(t-\tau) d \tau \\
& +\int_{0}^{+\infty} \widehat{\mathbf{L}}_{a, r}^{2}\left(\tau, t, \mathbf{u}_{r}^{1}(t-\tau), \mathbf{u}_{r}^{1}(t-\tau)\right) d \tau,
\end{aligned}
$$

$$
\begin{aligned}
\widehat{\mathbf{F}}_{r}^{2}(t)= & \left\{\widehat{\mathbf{K}}_{2, r}\left(t, \mathbf{u}_{r}^{1}(t)\right)+2{ }^{\mathrm{t}} \widehat{\mathbf{K}}_{2, r}\left(t, \mathbf{u}_{r}^{1}(t)\right)+\Delta \widehat{\mathbf{K}}_{e, r}(t)+\Delta \widehat{\mathbf{K}}_{g, r}(t)+2 \Delta \widehat{\mathbf{K}}_{2, r}\left(t, \mathbf{u}_{r}^{0}(t)\right)\right. \\
& \left.+2{ }^{\mathrm{t}} \Delta \widehat{\mathbf{K}}_{2, r}\left(t, \mathbf{u}_{r}^{0}(t)\right)\right\} \mathbf{u}_{r}^{1}(t)+\Delta \widehat{\mathbf{K}}_{2, r}\left(t, \mathbf{u}_{r}^{1}(t)\right) \mathbf{u}_{r}^{0}(t) \\
& +\int_{0}^{+\infty}\left\{\widehat{\mathbf{G}}_{2, r}\left(\tau, t, \mathbf{u}_{r}^{1}(t-\tau)\right)+{ }^{\mathrm{t}} \widehat{\mathbf{B}}_{2, r}\left(\tau, t, \mathbf{u}_{r}^{1}(t)\right)+\Delta \widehat{\mathbf{G}}_{r}(\tau, t)+\Delta \widehat{\mathbf{G}}_{2, r}\left(\tau, \mathbf{u}_{r}^{0}(t-\tau)\right)\right. \\
& \left.+\Delta \widehat{\mathbf{B}}_{2, r}\left(\tau, t, \mathbf{u}_{r}^{0}(t)\right)\right\} \mathbf{u}_{r}^{1}(t-\tau) d \tau+\int_{0}^{+\infty} \Delta \widehat{\mathbf{B}}_{2, r}\left(\tau, t, \mathbf{u}_{r}^{1}(t)\right) \mathbf{u}_{r}^{0}(t-\tau) d \tau
\end{aligned}
$$


Appendix G. Definition of the matrices for the perturbed nonlinear dynamical boundary value problem

$$
\begin{aligned}
& {\left[K_{1}\left(\Delta \mathbb{A}_{\overline{0}}\right)\right]_{d_{a i} d_{b j}} \circ \mathbf{X}_{0}^{r e f}=\delta_{a b}\left\{\left(\mathbb{K}_{1} \circ \mathbf{X}_{0}^{r e f}\right)::\left(\Delta \mathbb{A}_{\overline{0}} \circ \mathbf{X}_{0 \overline{0}}\right)\right\}_{i j},} \\
& {\left[K_{2}\left(\Delta \mathbb{G}_{\overline{0}}\right)\right]_{d_{a b} d_{c d}} \circ \mathbf{X}_{0}^{r e f}=\left\{\left(\mathbb{K}_{2} \circ \mathbf{X}_{0}^{r e f}\right)::\left(\Delta \mathbb{G}_{\overline{0}} \circ \mathbf{X}_{0 \overline{0}}\right)\right\}_{a b c d},}
\end{aligned}
$$

with for all $\mathbf{x}_{\overline{0}} \in \Omega_{\overline{0}}$, we note $\Delta \mathbb{A}_{\overline{0}}\left(\mathbf{x}_{\overline{0}}\right)=\lim _{\tau \rightarrow+\infty} \Delta \mathbb{G}_{\overline{0}}\left(\tau, \mathbf{x}_{\overline{0}}\right)$.

$$
\begin{aligned}
& {\left[T_{1}\left(\frac{\partial \mathbf{u}_{0 \overline{0}}}{\partial \mathbf{x}_{0}}\right)\right]_{d_{a i} d_{b j}} \circ \mathbf{X}_{0}^{r e f}=\delta_{a b}\left\{\left(\mathbb{T}_{1} \circ \mathbf{X}_{0}^{r e f}\right): \frac{\partial \mathbf{u}_{0 \overline{0}}}{\partial \mathbf{x}_{0}}\right\}_{i j},} \\
& {\left[T_{2}\left(\tau, \frac{\partial \mathbf{u}_{0 \overline{0}}}{\partial \mathbf{x}_{0}}\right)\right]_{d_{a b} d_{c d}} \circ \mathbf{X}_{0}^{r e f}=\left\{\left(\mathbb{T}_{2} \circ \mathbf{X}_{0}^{r e f}\right): \frac{\partial \mathbf{u}_{0 \overline{0}}}{\partial \mathbf{x}_{0}}\right\}_{a b c d} .} \\
& {\left[\Delta C_{e, r}\left(\tau, \mathbf{x}_{r}\right)\right]=\left[T_{2}\left(\tau, \frac{\partial \mathbf{u}_{0 \overline{0}}}{\partial \mathbf{x}_{0}}\right)\right]+\left[K_{2}\left(\Delta \mathbb{G}_{\overline{0}}\right)\right]+\left[C_{e, r}\left(\tau, \mathbf{x}_{r}\right)\right]\left(\operatorname{div}_{\mathbf{x}_{0}} \mathbf{u}_{0 \overline{0}}\right) \circ\left\{\mathbf{X}_{0}^{r e f}\right\}^{-1},} \\
& {\left[\Delta C_{g, r}\left(\mathbf{x}_{r}\right)\right]=\left[T_{1}\left(\frac{\partial \mathbf{u}_{0 \overline{0}}}{\partial \mathbf{x}_{0}}\right)\right]+\left[K_{1}\left(\Delta \mathbb{G}_{\overline{0}}\right)\right]+\left[C_{g, r}\left(\mathbf{x}_{r}\right)\right]\left(\operatorname{div}_{\mathbf{x}_{0}} \mathbf{u}_{0 \overline{0}}\right) \circ\left\{\mathbf{X}_{0}^{r e f}\right\}^{-1},} \\
& {\left[\Delta \widehat{C}_{g, r}\left(t, \mathbf{x}_{r}\right)\right]_{d_{a i} d_{b j}}=\frac{1}{2} \delta_{a b}\left\{\left[\Delta C_{e, r}\left(0, \mathbf{x}_{r}\right)\right]\left[B_{r}^{N L}\left(\mathbf{x}_{r}, \mathbf{U}^{\operatorname{tran}}(t)\right)\right] \mathbf{U}^{\operatorname{tran}}(t)\right\}_{d_{i j}}} \\
& +\delta_{a b}\left\{\left[\Delta C_{e, r}\left(0, \mathbf{x}_{r}\right)\right]\left[B_{r}\left(\mathbf{x}_{r}\right)\right] \mathbf{U}^{\operatorname{tran}}(t)\right\}_{d_{i j}} \\
& +\int_{0}^{+\infty} \delta_{a b}\left\{\left[\Delta \dot{C}_{e, r}\left(\tau, \mathbf{x}_{r}\right)\right]\left[B_{r}\left(\mathbf{x}_{r}\right)\right]\left\{\mathbf{U}^{\operatorname{tran}}(t-\tau)\right\}\right\}_{d_{i j}} d \tau \\
& +\frac{\delta_{a b}}{2} \int_{0}^{+\infty}\left\{\left[\Delta \dot{C}_{e, r}\left(\tau, \mathbf{x}_{r}\right)\right]\left[B_{r}^{N L}\left(\mathbf{x}_{r}, \mathbf{U}^{\text {tran }}(t-\tau)\right)\right] \mathbf{U}^{\operatorname{tran}}(t-\tau)\right\}_{d_{i j}} d \tau \text {. } \\
& {\left[C_{g, r}^{0}\left(\tau, \mathbf{x}_{r}\right)\right]_{d_{a i} d_{b j}}=\frac{1}{2} \delta_{a b}\left\{\left[C_{e, r}\left(0, \mathbf{x}_{r}\right)\right]\left[B_{r}^{N L}\left(\mathbf{x}_{r}, \mathbf{U}^{0}(t)\right)\right] \mathbf{U}^{0}(t)\right\}_{d_{i j}}} \\
& +\delta_{a b}\left\{\left[C_{e, r}\left(0, \mathbf{x}_{r}\right)\right]\left[B_{r}\left(\mathbf{x}_{r}\right)\right] \mathbf{U}^{0}(t)\right\}_{d_{i j}} \\
& +\int_{0}^{+\infty} \delta_{a b}\left\{\left[\dot{C}_{e, r}\left(\tau, \mathbf{x}_{r}\right)\right]\left[B_{r}\left(\mathbf{x}_{r}\right)\right]\left\{\mathbf{U}^{0}(t-\tau)\right\}\right\}_{d_{i j}} d \tau \\
& +\frac{\delta_{a b}}{2} \int_{0}^{+\infty}\left\{\left[\dot{C}_{e, r}\left(\tau, \mathbf{x}_{r}\right)\right]\left[B_{r}^{N L}\left(\mathbf{x}_{r}, \mathbf{U}^{0}(t-\tau)\right)\right] \mathbf{U}^{0}(t-\tau)\right\}_{d_{i j}} d \tau, \\
& {\left[\widehat{C}_{g, r}^{0}\left(\tau, \mathbf{x}_{r}\right)\right]_{d_{a i} d_{b j}}=\delta_{a b}\left\{\left[C_{e, r}\left(0, \mathbf{x}_{r}\right)\right]\left[B_{r}^{N L}\left(\mathbf{x}_{r}, \mathbf{U}^{\operatorname{tran}}(t)\right)\right] \mathbf{U}^{0}(t)\right\}_{d_{i j}}} \\
& +\delta_{a b} \int_{0}^{+\infty}\left\{\left[\dot{C}_{e, r}\left(\tau, \mathbf{x}_{r}\right)\right]\left[B_{r}^{N L}\left(\mathbf{x}_{r}, \mathbf{U}^{\text {tran }}(t-\tau)\right)\right] \mathbf{U}^{0}(t-\tau)\right\}_{d_{i j}} d \tau,
\end{aligned}
$$




$$
\begin{aligned}
& {\left[K_{g, r}^{0}(t)\right]=\int_{\Omega_{r}}\left[B_{r}\left(\mathbf{x}_{r}\right)\right]^{\mathrm{T}}\left[C_{g, r}^{0}\left(\mathbf{x}_{r}\right)\right]\left[B_{r}\left(\mathbf{x}_{r}\right)\right] d \mathbf{x}_{r}} \\
& {\left[\widehat{K}_{g, r}^{0}(t)\right]=\int_{\Omega_{r}}\left[B_{r}\left(\mathbf{x}_{r}\right)\right]^{\mathrm{T}}\left[\widehat{C}_{g, r}^{0}\left(\mathbf{x}_{r}\right)\right]\left[B_{r}\left(\mathbf{x}_{r}\right)\right] d \mathbf{x}_{r},} \\
& {\left[K_{r}^{0}(t)\right]=\left[K_{g, r}^{0}(t)\right]+\left[K_{2, r}\left(\mathbf{U}^{0}(t)\right)\right]+\left[K_{2, r}\left(\mathbf{U}^{0}(t)\right)\right]^{\mathrm{T}}+2\left[K_{3, r}\left(\mathbf{U}^{0}(t), \mathbf{U}^{0}(t)\right)\right],} \\
& {\left[\widehat{K}_{r}^{0}(t)\right]=\left[\widehat{K}_{g, r}^{0}(t)\right]+\left[\widehat{K}_{e, r}(t)\right]+\left[\widehat{K}_{g, r}(t)\right]+2\left[\widehat{K}_{2, r}\left(t, \mathbf{U}^{0}(t)\right)\right]+2\left[\widehat{K}_{2, r}\left(t, \mathbf{U}^{0}(t)\right)\right]^{\mathrm{T}},(206)} \\
& {\left[G_{r}^{0}(\tau, t)\right]=\left[G_{r}(\tau)\right]+2\left[G_{2, r}\left(\tau, \mathbf{U}^{0}(t-\tau)\right)\right]+2\left[G_{3, r}\left(\tau, \mathbf{U}^{0}(t), \mathbf{U}^{0}(t-\tau)\right)\right],} \\
& {\left[\widehat{G}_{r}^{0}(\tau, t)\right]=\left[\widehat{G}_{r}(\tau, t)\right]+2\left[\widehat{G}_{2, r}\left(\tau, t, \mathbf{U}^{0}(t-\tau)\right)\right]+\left[\widehat{B}_{2, r}\left(\tau, t, \mathbf{U}^{0}(t)\right)\right],} \\
& {\left[\Delta K_{c, r}\right]=\int_{\Omega_{r}} \Delta \rho_{r}\left[N_{r}\left(\mathbf{x}_{r}\right)\right]^{\mathrm{T}}[R]^{2}\left[N_{r}\left(\mathbf{x}_{r}\right)\right] d \mathbf{x}_{r},} \\
& {\left[\Delta K_{g, r}\right]=\int_{\Omega_{r}}\left[B_{r}\left(\mathbf{x}_{r}\right)\right]^{\mathrm{T}}\left[\Delta C_{g, r}\left(\mathbf{x}_{r}\right)\right]\left[B_{r}\left(\mathbf{x}_{r}\right)\right] d \mathbf{x}_{r}} \\
& {\left[\Delta K_{e, r}\right]=\int_{\Omega_{r}}\left[B_{r}\left(\mathbf{x}_{r}\right)\right]^{\mathrm{T}}\left[\Delta C_{e, r}\left(0, \mathbf{x}_{r}\right)\right]\left[B_{r}\left(\mathbf{x}_{r}\right)\right] d \mathbf{x}_{r},} \\
& {\left[\Delta K_{2, r}(\mathbf{V})\right]=\int_{\Omega_{r}}\left[B_{r}\left(\mathbf{x}_{r}\right)\right]^{\mathrm{T}}\left[\Delta C_{e, r}\left(0, \mathbf{x}_{r}\right)\right]\left[B_{r}^{N L}\left(\mathbf{x}_{r}, \mathbf{V}\right)\right] d \mathbf{x}_{r},} \\
& {\left[\Delta K_{3, r}(\mathbf{W}, \mathbf{V})\right]=\frac{1}{2} \int_{\Omega_{r}}\left[B_{r}^{N L}\left(\mathbf{x}_{r}, \mathbf{W}\right)\right]^{\mathrm{T}}\left[\Delta C_{e, r}\left(0, \mathbf{x}_{r}\right)\right]\left[B_{r}^{N L}\left(\mathbf{x}_{r}, \mathbf{V}\right)\right] d \mathbf{x}_{r},} \\
& {\left[\Delta M_{r}\right]=\int_{\Omega_{r}} \Delta \rho_{r}\left[N_{r}\left(\mathbf{x}_{r}\right)\right]^{\mathrm{T}}\left[N_{r}\left(\mathbf{x}_{r}\right)\right] d \mathbf{x}_{r},} \\
& {\left[\Delta C_{r}\right]=2 \int_{\Omega_{r}} \Delta \rho_{r}\left[N_{r}\left(\mathbf{x}_{r}\right)\right]^{\mathrm{T}}[R]\left[N_{r}\left(\mathbf{x}_{r}\right)\right] d \mathbf{x}_{r},} \\
& {\left[\Delta G_{r}(t)\right]=\int_{\Omega_{r}}\left[B_{r}\left(\mathbf{x}_{r}\right)\right]^{\mathrm{T}}\left[\Delta \dot{C}_{e, r}\left(t, \mathbf{x}_{r}\right)\right]\left[B_{r}\left(\mathbf{x}_{r}\right)\right] d \mathbf{x}_{r},} \\
& {\left[\Delta G_{2, r}(t, \mathbf{V})\right]=\int_{\Omega_{r}}\left[B_{r}\left(\mathbf{x}_{r}\right)\right]^{\mathrm{T}}\left[\Delta \dot{C}_{e, r}\left(t, \mathbf{x}_{r}\right)\right]\left[B_{r}^{N L}\left(\mathbf{x}_{r}, \mathbf{V}\right)\right] d \mathbf{x}_{r},} \\
& {\left[\Delta G_{3, r}(t, \mathbf{W}, \mathbf{V})\right]=\frac{1}{2} \int_{\Omega_{r}}\left[B_{r}^{N L}\left(\mathbf{x}_{r}, \mathbf{W}\right)\right]^{\mathrm{T}}\left[\Delta \dot{C}_{e, r}\left(t, \mathbf{x}_{r}\right)\right]\left[B_{r}^{N L}\left(\mathbf{x}_{r}, \mathbf{V}\right)\right] d \mathbf{x}_{r},} \\
& {\left[\Delta \widehat{K}_{g, r}(t)\right]=\int_{\Omega_{r}}\left[B_{r}\left(\mathbf{x}_{r}\right)\right]^{\mathrm{T}}\left[\Delta \widehat{C}_{g, r}\left(t, \mathbf{x}_{r}\right)\right]\left[B_{r}\left(\mathbf{x}_{r}\right)\right] d \mathbf{x}_{r},} \\
& {\left[\Delta \widehat{K}_{e, r}(t)\right]=\left[\Delta K_{2, r}\left(\mathbf{U}^{\operatorname{tran}}(t)\right)\right]+\left[\Delta K_{2, r}\left(\mathbf{U}^{\operatorname{tran}}(t)\right)\right]^{\mathrm{T}}} \\
& +2\left[\Delta K_{3, r}\left(\mathbf{U}^{\operatorname{tran}}(t), \mathbf{U}^{\operatorname{tran}}(t)\right)\right], \\
& {\left[\Delta \widehat{K}_{2, r}(t, \mathbf{V})\right]=\left[\Delta K_{3, r}\left(\mathbf{U}^{\operatorname{tran}}(t), \mathbf{V}\right)\right],} \\
& {\left[\Delta \widehat{G}_{r}(\tau, t)\right]=\left[\Delta G_{2, r}\left(\tau, \mathbf{U}^{\text {tran }}(t)\right)\right]^{\mathrm{T}}+\left[\Delta G_{2, r}\left(\tau, \mathbf{U}^{\text {tran }}(t-\tau)\right)\right]} \\
& +2\left[\Delta G_{3, r}\left(\tau, \mathbf{U}^{\operatorname{tran}}(t), \mathbf{U}^{\operatorname{tran}}(t-\tau)\right)\right], \\
& {\left[\Delta \widehat{B}_{2, r}(\tau, t, \mathbf{V})=2\left[\Delta G_{3, r}\left(\tau, \mathbf{V}, \mathbf{U}^{\operatorname{tran}}(t-\tau)\right)\right],\right.} \\
& {\left[\Delta \widehat{G}_{2, r}(\tau, t, \mathbf{V})\right]=\left[\Delta G_{3, r}\left(\tau, \mathbf{U}^{\operatorname{tran}}(t), \mathbf{V}\right)\right],} \\
& {\left[\widehat{L}_{a, h}^{1}(\tau, t)\right]_{i j}=<\widehat{\mathbf{L}}_{a, r}^{1} \mathbf{g}_{i}, \mathbf{g}_{j}>,} \\
& {\left[K_{r}\right]=\left[K_{e, r}\right]+\left[K_{g, r}\right]+\left[K_{c, r}\right],}
\end{aligned}
$$




$$
\begin{aligned}
& \left\{\Delta \widehat{\mathbf{L}}_{a, h}\right\}_{j}=<\Delta \widehat{\mathbf{L}}_{a, r}, \mathbf{g}_{j}> \\
& \left\{\Delta \widehat{\mathbf{L}}_{r, h}(t)\right\}_{j}=<\Delta \widehat{\mathbf{L}}_{r}(t), \mathbf{g}_{j}> \\
& \left\{\Delta \mathbf{L}_{r, h}(t)\right\}_{j}=<\Delta \mathbf{L}_{r}(t), \mathbf{g}_{j}>, \\
& \left\{\widehat{\mathbf{L}}_{a, h}^{2}(\tau, t, \mathbf{U}, \mathbf{V})\right\}_{j}=<\widehat{\mathbf{L}}_{a, h}^{2}\left(\tau, t,\left[N_{r}\left(\mathbf{x}_{r}\right)\right] \mathbf{U},\left[N_{r}\left(\mathbf{x}_{r}\right)\right] \mathbf{V}\right), \mathbf{g}_{j}>,
\end{aligned}
$$

and

$$
\begin{gathered}
\mathbf{F}_{r, h}^{1}(t)=\left[\Delta M_{r}\right] \ddot{\mathbf{U}}_{t}^{0}+\left[\Delta C_{r}\right] \dot{\mathbf{U}}_{t}^{0}+\left\{\left[\Delta K_{e, r}\right]+\left[\Delta K_{g, r}\right]+\left[\Delta K_{c, r}\right]+\frac{1}{2}\left[\Delta K_{2, r}\left(\mathbf{U}_{t}^{0}\right)\right]\right. \\
\left.+\left[\Delta K_{2, r}\left(\mathbf{U}_{t}^{0}\right)\right]^{\mathrm{T}}+\left[\Delta K_{3, r}\left(\mathbf{U}_{t}^{0}, \mathbf{U}_{t}^{0}\right)\right]\right\} \mathbf{U}_{t}^{0} \\
+\int_{0}^{+\infty}\left\{\frac{1}{2}\left[\Delta G_{2, r}\left(\tau, \mathbf{U}_{t-\tau}^{0}\right)\right]+\left[\Delta G_{2, r}\left(\tau, \mathbf{U}_{t}^{0}\right)\right]^{\mathrm{T}}+\left[\Delta G_{3, r}\left(\tau, \mathbf{U}_{t}^{0}, \mathbf{U}_{t-\tau}^{0}\right)\right]\right. \\
\left.+\left[\Delta G_{r}(\tau)\right]\right\} \mathbf{U}_{t-\tau}^{0} d \tau-\Delta \widehat{\mathbf{L}}_{a, h}-\Delta \mathbf{L}_{r, h}(t)
\end{gathered}
$$

$$
\begin{aligned}
\widehat{\mathbf{F}}_{r, h}^{1}(t) & =\left\{\left[\Delta \widehat{K}_{g, r}(t)\right]+\left[\Delta \widehat{K}_{e, r}(t)\right]+\left[\Delta \widehat{K}_{2, r}\left(t, \mathbf{U}_{t}^{0}\right)\right]+2\left[\Delta \widehat{K}_{2, r}\left(t, \mathbf{U}_{t}^{0}\right)\right]^{\mathrm{T}}\right\} \mathbf{U}_{t}^{0} \\
+ & \int_{0}^{+\infty}\left\{\left[\Delta \widehat{G}_{2, r}\left(\tau, t, \mathbf{U}_{t-\tau}^{0}\right)\right]+\left[\Delta \widehat{G}_{r}(\tau, t)\right]+\left[\Delta \widehat{B}_{2, r}\left(\tau, t, \mathbf{U}_{t}^{0}\right)\right]\right\} \mathbf{U}_{t-\tau}^{0} d \tau+\Delta \widehat{\mathbf{L}}_{r, h}(t),
\end{aligned}
$$

$$
\begin{aligned}
\mathbf{F}_{r, h}^{2}(t) & =\left[\Delta M_{r}\right] \ddot{\mathbf{U}}_{t}^{1}+\left[\Delta C_{r}\right] \dot{\mathbf{U}}_{t}^{1}+\left\{\left[\Delta K_{3, r}\left(\mathbf{U}_{t}^{1}, \mathbf{U}_{t}^{0}\right)\right]+\left[\Delta K_{2, r}\left(\mathbf{U}_{t}^{1}\right)\right]^{\mathrm{T}}\right\} \mathbf{U}_{t}^{0} \\
+ & \left\{\frac{1}{2}\left[K_{2, r}\left(\mathbf{U}_{t}^{1}\right)\right]+\left[K_{2, r}\left(\mathbf{U}_{t}^{1}\right)\right]^{\mathrm{T}}+2\left[K_{3, r}\left(\mathbf{U}_{t}^{1}, \mathbf{U}_{t}^{0}\right)\right]+\left[K_{3, r}\left(\mathbf{U}_{t}^{0}, \mathbf{U}_{t}^{1}\right)\right]+\left[\Delta K_{e, r}\right]\right. \\
& \left.+\left[\Delta K_{g, r}\right]+\left[\Delta K_{c, r}\right]+\left[K_{2, r}\left(\mathbf{U}_{t}^{0}\right)\right]+\left[\Delta K_{2, r}\left(\mathbf{U}_{t}^{0}\right)\right]^{\mathrm{T}}+2\left[\Delta K_{3, r}\left(\mathbf{U}_{t}^{0}, \mathbf{U}_{t}^{0}\right)\right]\right\} \mathbf{U}_{t}^{1} \\
+ & \int_{0}^{+\infty}\left\{\frac{1}{2}\left[G_{2, r}\left(\tau, \mathbf{U}_{t-\tau}^{1}\right)\right]+\left[G_{2, r}\left(\tau, \mathbf{U}_{t}^{1}\right)\right]^{\mathrm{T}}+2\left[G_{3, r}\left(\tau, \mathbf{U}_{t}^{1}, \mathbf{U}_{t-\tau}^{0}\right)\right]+\left[\Delta G_{r}(\tau)\right]\right. \\
& \left.\quad+\left[G_{3, r}\left(\tau, \mathbf{U}_{t}^{0}, \mathbf{U}_{t-\tau}^{1}\right)\right]+2\left[\Delta G_{2, r}\left(\tau, \mathbf{U}_{t-\tau}^{0}\right)\right]+2\left[\Delta G_{3, r}\left(\tau, \mathbf{U}_{t}^{0}, \mathbf{U}_{t-\tau}^{0}\right)\right]\right\} \mathbf{U}_{t-\tau}^{1} d \tau \\
+ & \int_{0}^{+\infty}\left\{\left[\Delta G_{2, r}\left(\tau, \mathbf{U}_{t}^{1}\right)\right]^{\mathrm{T}}+\left[\Delta G_{3, r}\left(\tau, \mathbf{U}_{t}^{1}, \mathbf{U}_{t-\tau}^{0}\right)\right]\right\} \mathbf{U}_{t-\tau}^{0} d \tau \\
+ & \int_{0}^{+\infty} \widehat{\mathbf{L}}_{a, h}^{2}\left(\tau, t, \mathbf{U}_{t-\tau}^{1}, \mathbf{U}_{t-\tau}^{1}\right) d \tau,
\end{aligned}
$$

$$
\begin{aligned}
\widehat{\mathbf{F}}_{r, h}^{2}(t)= & \left\{\left[\widehat{K}_{2, r}\left(t, \mathbf{U}_{t}^{1}\right)\right]+2\left[\widehat{\mathbf{K}}_{2, r}\left(t, \mathbf{U}_{t}^{1}\right)\right]^{\mathrm{T}}+\left[\Delta \widehat{K}_{e, r}(t)\right]+\left[\Delta \widehat{\mathbf{K}}_{g, r}(t)\right]\right. \\
& \left.+2\left[\Delta \widehat{K}_{2, r}\left(t, \mathbf{U}_{t}^{0}\right)\right]+2\left[\Delta \widehat{K}_{2, r}\left(t, \mathbf{U}_{t}^{0}\right)\right]^{\mathrm{T}}\right\} \mathbf{U}_{t}^{1}+\left[\Delta \widehat{K}_{2, r}\left(t, \mathbf{U}_{t}^{1}\right)\right] \mathbf{U}_{t}^{0} \\
+ & \int_{0}^{+\infty}\left\{\left[\widehat{G}_{2, r}\left(\tau, t, \mathbf{U}_{t-\tau}^{1}\right)\right]+\left[\widehat{B}_{2, r}\left(\tau, t, \mathbf{U}_{t}^{1}\right)\right]^{\mathrm{T}}+\left[\Delta \widehat{G}_{r}(\tau, t)\right]+\left[\Delta \widehat{G}_{2, r}\left(\tau, \mathbf{U}_{t-\tau}^{0}\right)\right]\right. \\
& \left.+\left[\Delta \widehat{B}_{2, r}\left(\tau, t, \mathbf{U}_{t}^{0}\right)\right]\right\} \mathbf{U}_{t-\tau}^{1}+\int_{0}^{+\infty}\left[\Delta \widehat{B}_{2, r}\left(\tau, t, \mathbf{U}_{t-\tau}^{0}\right)\right] \mathbf{U}_{t-\tau}^{0} d \tau .
\end{aligned}
$$




\section{References}

[1] V. Arnold, Mathematical Methods of Calssical Mechanics, Springer, New York, (1978).

[2] K. J. Bathe, Finite Element Procedures in Engineering Analysis, Prentice-Hall., Englewood Cliffs, New Jersey, (1982).

[3] O.A. Bauchau, A Solution of the Eigenproblem for Undamped Gyroscopic Systems with the Lanczos Algorithm, Int. Journal for Numerical Methods in Engineering , 23, 1705-1713.

[4] C.E. Beevers, Some Continuous Dependence Results in the Linear Dynamic Theory of Anisotropic Viscoelasticity, Journal de Mécanique (1975), 14 (4), 640-651.

[5] N. N. Bogolubov, I.A. Mitropol'Skij, Les Méthodes asymptotiques en théorie des oscillations non linéaires., Paris, Gauthier Villars, 1962.

[6] R. Bouc, G. Geymonat, M. Jean, B. Nayroles, Solide viscoélastique, Journal de mécanique (1975), 14 (4), 612-637.

[7] H. Brezis, Analyse fonctionnelle, théorie et applications, Paris, Masson, 1993.

[8] J. Casey, P. M. Naghdi, Physically NonLinear and Related Approximate Theories of Elasticity and their Invariance Properties, Archive for Rational Mechanics and Analysis (1985), 88, 59-82

[9] J. Casey, P. M. Naghdi, An Invariant Infinitesimal Theory of Motions Superposed on a given Motion, Archive for Rational Mechanics and Analysis (1985), 76, 355-391

[10] G. Chakraborty, A.K. Mallik, H. Hatwl, Non-Linear Vibration of a Travelling Beam, Int. J. Non-Linear Mechanics, 345(1999), 655-670

[11] P. G. Ciarlet, Mathematical Elasticity, North-Holland, Amsterdam, 1988, 2 volumes.

[12] P. G. Ciarlet, The Finite Element Method for Elliptic Problems, North-Holland, Amsterdam, 1978.

[13] B.D. Coleman, W. Noll, Foundations of Linear Viscoelasticity, Reviews of Modern Physics (1961), 33 (2), $239-249$.

[14] R. Dautray, J. L. Lions, Mathematical Analysis and Numerical Methods for Science and Technology, Springer-Verlag, Berlin, 1992.

[15] C. Desceliers, Dynamique non linéaire en déplacements finis des structures tridimensionnelles viscoélastiques en rotation, Thése de Doctorat, Ecole Centrale Paris, 2001.

[16] S. Dubigeon, J. C. Michon, Modes for Deformable Periodic Cyclic Symmetric Systems Driven in Uniform Rotation by a Flexible Shaft, Journal of Sound and Vibration (1986), 106 (1), 53-70.

[17] G. Duvaut, J. L Lions, Les inéquations en mécanique et en physique, Dunod, Paris, 1972. 
[18] E. Esmailzadeh, G. Nakhaie-Jazar, Periodic behavior of a Cantilever Beam with End Mass Subjected to Harmonic Base Excitation, Int. J. Non-Linear Mechanics, 4, 33, 567-577, 1998

[19] M. Géradin, D. Rixen, Mechanical Vibrations, Wiley, Chichester, 1994.

[20] P. Germain, Cours de mécanique des milieux continus, Masson, Paris, 1973.

[21] K. K. Gupta, Development of a Unified Numerical Procedure for Free Vibration Analysis of Structures, Int. Journal for Numerical Methods in Engineering (1981), 17, 187-198.

[22] A. E. Green, R. S. Rivlin, R. T. Shield, General Theory of Small Elastic Deformations Superposed on Finite Elastic Deformations, Archive for Rational Mechanics and Analysis (1951), 211, 128-154

[23] T. J. R. Hughes, The Finite Element Method, Prentice Hall, Englewood Cliffs, New Jersey (1987)

[24] G. Jacquet-Richardet, G. Ferraris, P. Rieutord, Frequencies and Modes of Rotating Flexible Bladed Disc-Shaft Assemblies : A Global Cyclic Symmetry Approach, Journal of Sound and Vibration (1996), 191 (5), p. 901-915.

[25] L. Jézéquel, C. H. Lamarque, Analysis of Non-linear Dynamical Systems by the Normal Form Theory, Journal of Sound and Vibration (1991), 149 (3), 429-459.

[26] L. Jézéquel, Extention des méthodes de synthèse modale au cas non linéaire, Revue Française de Mécanique (1987), 3, 159-172.

[27] P. B. Kahn, Y. Zarmi, Nonlinear Dynamics Exploration Through Normal Forms, A Wiley-Interscience Publication, John Wiley and Sons, New York, 1998.

[28] M. J. Kruse, C. Pierre, An Experimental Investigation of Vibration Localization in Bladed Disks, Part I : Free Response, Part II : Forced Response, ASME Paper 97-GT-502-Proceedings of ASME Gas Turbine Conference, Orlando, Florida juin 1997.

[29] K. R. Meyer, G. R. Hall, Introduction to Hamiltonian Dynamical Systems and the N-Body Problem, Springer-Verlag, Nex York, 1992.

[30] A. Y. T. Leung, Q. C. Zhang, Higher Order Normal Form and Period Averaging, Journal of Sound and Vibration (1998), 217 (5), 795-806

[31] J. Mandel, Cours de Mécanique des milieux continus, Tomes II, Mécanique des solides. (1966), Gauthier-Villars, Paris.

[32] R. J. McDonald, J. Murdock, N. Namachchivaja Sri, Non-Autonomous Normal Forms for Hamiltonian Systems, Dynamics and Stability of Systems (1999), 14 (4), 357-384.

[33] W. Nagata, N.S. Namachchivaya, Bifurcations in Gyroscopic Systems With an Application to Rotating Shafts, Proc. R. Soc. Lond. A. (1998), 454, 543-585.

[34] A. H. Nayfeh, Method of Normal Forms, A Wiley-Interscience Publication, John Wiley and Sons, New York, 1993. 
[35] A. H. Nayfeh, Introduction to perturbation techniques, Wiley, New York, 1981.

[36] A. H. Nayfeh, B. Balachandran, Applied Nonlinear Dynamics, Wiley-Interscience Publication, New York, 1995.

[37] J. T. Oden, J. N. Reddy, Variational Methods in Theoritical Mechanics, Springer Verlag, New York, 1983.

[38] R. Ohayon, C. Soize, Structural Acoustics and Vibration, Academic Press, London, 1998.

[39] O. M. O'Reilly, J. S. Turcotte, Elastic Rods with Moderate Rotation, Journal of Elasticity (1997), 48, 193-216

[40] D. Qian, J. S. Hansen, A Substructure Synthesis Method for Non-Symmetric Viscoelastic Systems, Journal of Sound and Vibration (1995), 185 (4), 627-641.

[41] M. Reed, B. Simon, Methods of Modern Mathematical Physics, Academic Press, New York, 1980.

[42] C. Truesdell, Introduction à la mécanique rationnelle des milieux continus, Masson, Paris, 1974.

[43] C. Truesdell, Mechanics of Solids, Vol III, Theory of Viscoelasticity, Plasticity, Elasticity Waves and Elastic Stability., Springer-Verlag, Berlin, 1984.

[44] W. Wang, J. Kirkhope, New Eigensolutions and Modal Analysis for Gyroscopic / Rotor Systems, Part 1 : Undamped Systems, Journal of Sound and Vibration (1994), 175 (2), 159-170.

[45] W. Wang, J. Kirkhope, New Eigensolutions and Modal Analysis for Gyroscopic / Rotor Systems, Part 2 : Perturbation Analysis for Damped Systems, Journal of Sound and Vibration (1994), 175 (2), 171-183.

[46] J. A. Wickert, Transient Vibration of Gyroscopic System with unsteady superposed motion, Journal of Sound and Vibration (1996), 195, 797-807.

[47] A. Wineman, R. Kolberg, Response of Beams of Non-Linear Viscoelastic Materials Exhibiting Strain-dependent Stress Relaxation, Int. J. Non-Linear Mechanics, Vol 195, N.5, 863-883

[48] H. H. Yoo, S. H. Shin, Vibration Analysis of Rotating Cantilever Beams, Journal of Sound and Vibration (1998), 212 (5), 807-828.

[49] A. A. Zevin, On the Theory of Linear Gyroscopic Systems, J. Appl. Maths Mechs (1996), 60 (2), 227-232.

[50] O. C. Zienkiewicz, R. L. Taylor, The Finite Element Method, McGraw-Hill Book Company, 1991, 2 volumes. 